\title{
Les myriapodes chilopodes (Chilopoda) du Parc national du Mercantour, du département des Alpes-Maritimes et de leurs environs : description d'une nouvelle espèce du genre Lithobius Leach, 1814 s.s., synthèse des connaissances et espèces menacées
}

\author{
Étienne IORIO \\ Groupe d'étude des Invertébrés armoricains (GRETIA), \\ 5, rue du général Leclerc, F-44390 Nort-sur-Erdre (France) \\ e.iorio@gretia.org \\ Marzio ZAPPAROLI \\ Dipartimento per la Innovazione nei sistemi Biologici, \\ Agroalimentari e Forestali (DIBAF), \\ Università degli Studi della Tuscia, \\ Via San Camillo de Lellis snc, I-01100 Viterbo (Italy) \\ zapparol@unitus.it
}

Philippe PONEL Institut méditerranéen de Biodiversité et d'Écologie (IMBE-CNRS), Aix-Marseille Université-Univ. Avignon-IRD, Technopôle de l'Environnement Arbois-Méditerranée, BP 80, F-13545 Aix-en-Provence Cedex 04 (France) philippe.ponel@imbe.fr

Jean-Jacques GEOFFROY Muséum national d'Histoire naturelle, Département Écologie \& Gestion de la Biodiversité, UMR7204 CESCO CNRS-MNHN-UPMC, 4, avenue du Petit Château, F-91800 Brunoy (France) geoffroy@mnhn.fr

Publié le 27 mars 2015

Iorio E., Zapparoli M., Ponel P. \& Geoffroy J.-J. 2015. - Les myriapodes chilopodes (Chilopoda) du Parc national du Mercantour, du département des Alpes-Maritimes et de leurs environs : description d'une nouvelle espèce du genre Lithobius Leach, 1814 s.s., synthèse des connaissances et espèces menacées, in Daugeron C., Deharveng L., Isaia M., Villemant C. \& Judson M. (coords), Mercantour/Alpi Marittime All Taxa Biodiversity Inventory. Zoosystema 37 (1): $211-$ 238. http://dx.doi.org/10.5252/z2015n1a11

RÉSUMÉ

Suite à l'examen de nombreux spécimens, les auteurs exposent des données nouvelles sur les chilopodes du Parc national du Mercantour, du département des Alpes-Maritimes et de leurs environs. Ces observations améliorent la connaissance de la répartition de certaines espèces rares et localisées et permettent en outre de décrire une nouvelle espèce en provenance d'Entrevaux (Alpes-de-Haute-Provence) : Lithobius (Lithobius) lemairei n. sp. Cette remarquable nouvelle espèce a été trouvée dans un milieu souterrain superficiel de type MSS et vit potentiellement plus en profondeur dans un système de grottes ou de crevasses; elle possède des caractères troglomorphes adaptés à son mode de vie, notamment une extrême réduction des ocelles et une augmentation de la taille de l'organe de Tömösvàry. Par ailleurs, elle possède des sillons latéraux-internes sur les fémurs, tibias, basitarses et tarses des pattes 15 , et secondairement 


\section{MOTS CLÉS \\ France, \\ répartition, \\ écologie, \\ géophilomorphes
littoraux \\ conservation, \\ inventaire.}

KEY WORDS

France,

distribution ecology,

littoral geophilomorphs,

conservation, inventory. sur les mêmes articles des pattes 14. Ces structures en font une espèce particulièrement distincte de toutes les autres appartenant au genre Lithobius Leach, 1814 dans ce secteur géographique. Suite à un réexamen des spécimens en provenance des Alpes-Maritimes, Eupolybothrus (Eupolybothrus) fasciatus (Newport, 1845) est à retirer de la faune de France. Nos recherches ciblées sur les espèces halophiles et halobies de géophilomorphes du littoral des Alpes-Maritimes montrent que ces espèces ont au moins fortement régressé dans cette aire géographique. Une synthèse écologique est effectuée sur les espèces recensées, cumulant les nouvelles connaissances et les contributions antérieures sur ce département. Une liste des chilopodes recensés dans le Parc national du Mercantour et/ou dans le département des Alpes-Maritimes est proposée. Elle inclut des informations sur l'endémisme au niveau local et/ou au niveau national pour les taxa concernés, sur l'éventuelle présence en limite d'aire de répartition, ainsi que d'éventuelles particularités écologiques remarquables (troglobies, halobies, orophiles). Certains endémiques locaux et certaines espèces écologiquement très spécialisées apparaissent comme potentiellement menacés, voire en fort déclin avéré suite à nos observations. Une première liste de ces espèces, qualifiées ici de «sensibles", est proposée.

\begin{abstract}
The centipedes (Chilopoda) of the Mercantour National Park, of the Alpes-Maritimes department and their surroundings: new data and description of a new species of genus Lithobius Leach, 1814 s.s., biogeographic knowledge and threatened species.

After the examination of many specimens, the authors give new data on centipedes of the Mercantour National Park, the Alpes-Maritimes department and their surroundings. These examinations improve the knowledge of the distribution of several fairly rare species and allow to describe a remarkable new species from Entrevaux (Alpes-de-Haute-Provence department): Lithobius (Lithobius) lemairei n. sp. This new species occurs in superficial subterranean environments (MSS) and maybe deeper in caves or crevices; it exhibits morphological troglomorphic adaptations such as reduction of ocelli and an increased size of the Tömösváry's organ. It also has lateral-internal sulci on femora, tibiae, tarsi one and two of the 15th legs, and secondarily on the same articles of the 14th legs. These traits clearly distinguish this species from the others belonging to the genus Lithobius Leach, 1814 in this geographical area. After a reexamination of the published records and of the specimens recorded from the Alpes-Maritimes, Eupolybothrus (Eupolybothrus) fasciatus (Newport, 1845) must to be removed from the French. After our research of the halophilous and halobiontic species of geophilomorphs on the littoral of Alpes-Maritimes, it appears that these species have at least strongly declined in this area. An ecological synthesis is done on all the observed species, by cumulating the new data and the preceding contributions on the Alpes-Maritimes. A checklist of the centipedes known in the Mercantour National Park and/or in the Alpes-Maritimes department is given. It includes data on: 1) endemism at local and/or national level(s);2) their presence in their limits of distribution; and 3) some remarkable ecological particularities (troglobiontic, halobiontic, orophilous species). Some local endemics and some stenoecious species seem to be potentially threatened, or even in a decline according to our observations. A preliminary list of these " vulnerables species » is proposed.
\end{abstract}

\section{INTRODUCTION}

Les Alpes maritimes constituent un des dix « hotspots " régionaux de biodiversité des plantes pour la zone méditerranéenne et, à une échelle plus fine, le plus important centre d'endémisme de l'arc alpin (Médail \& Quézel 1997a, 1999; Médail \& Diadéma 2006). L'intérêt de cette aire géographique est évident depuis longtemps pour de nombreux groupes d'arthropodes (Billi et al. 2011). Aussi bien sur le plan floristique que faunistique, il s'explique par une topographie morcelée, des variations climatiques très contrastées sur de courtes distances et des milieux très hétérogènes sur une superficie réduite, mais aussi par l'existence de nombreux refuges glaciaires. De nombreuses espèces, épigées comme souterraines, s'y trouvent en limite de répartition occidentale ou orientale, voire y sont strictement endémiques.
Conscients de cet intérêt depuis la première moitié du XIXe siècle, de nombreux auteurs ont contribuéà la connaissance des chilopodes du département des Alpes-Maritimes et de ses abords immédiats, à l'aide de travaux de nature et d'ampleur diverses. Risso (1826) fut le premier à citer, dans le cinquième tome de son histoire naturelle sur les environs de Nice et des Alpes-Maritimes, quatre myriapodes nouveaux pour le département, dont trois espèces nouvelles, parmi lesquelles seul Eupolybothrus longicornis Risso, 1826 demeure valide aujourd'hui. Ce travail fut suivi par d'autres contributions mineures sur le groupe dans ce secteur: Walckenaer \& Gervais (1847), qui citèrent deux espèces déjà trouvées par Risso, mais dans une nouvelle localité; Koch (1862), qui reporta une espèce et une localité déjà citées par les auteurs précédents; Pocock (1890), qui ajouta huit espèces supplémentaires dans une nouvelle localité. 
Le $\mathrm{XX}^{\mathrm{e}}$ siècle vit paraître des travaux plus importants, qui ont considérablement amélioré la connaissance de la classe Chilopoda Latreille, 1817 dans les Alpes-Maritimes, notamment ceux de Brölemann (1904) et Caziot (1925). Le second auteur a établi une liste fournie d'espèces, basée surtout sur des prospections au niveau du littoral et reprenant les espèces monégasques citées par Brölemann (1904). D'autres travaux intéressant cette aire et s'étendant jusqu' en 2000 sont à signaler (Brölemann 1901, 1925, 1930; Chalande \& Ribaut 1909; Brölemann \& Ribaut 1911, 1912; Caziot 1915; Ribaut 1915; Verhoeff 1925, 1928, 1931, 1934; Jeannel 1926; Attems 1929, 1930, 1947; Manfredi 1932; Wolf 1938; Demange 1955 ; Matic 1960 ; Aellen 1984 ; Bologna \& Vigna Taglianti 1985; Minelli 1985; Delaunay 2000). À la fin du XXe siècle, deux auteurs italiens se penchèrent sur la faune des Alpes maritimes au sens large, faisant part de prospections dans de nouvelles localités françaises, notamment montagnardes (Zapparoli 1980; Minelli \& Zapparoli 1985, 1992). Ces travaux permirent d'ajouter plusieurs espèces inédites pour le département et pour la France.

Le XXIe siècle marqua le début d'une reprise des recherches sur les chilopodes dans le secteur concerné et d'autres adjacents (Stoev \& Geoffroy 2004; Iorio \& Minelli 2005; Bonato et al. 2006; Iorio \& Berg 2007; Iorio \& Geoffroy 2007a, b, c, 2008; Iorio 2012). La connaissance des chilopodes des Alpes-Maritimes connut un développement notable grâce à l'ouvrage de Iorio (2008), qui fournit près de 1500 données nouvelles sur la distribution géographique des chilopodes de ce département et deux espèces supplémentaires pour la France, ainsi qu'à des dizaines d'autres données sur les Lithobiomorpha (Iorio 2010b) et à la description de deux taxa nouveaux pour la science parmi les Scolopendromorpha Pocock, 1895 et Geophilomorpha Pocock, 1895 (Iorio 2010a; Bonato et al. 2011): Cryptops umbricus lewisi Iorio, 2010 et Clinopodes vesubiensis Bonato, Iorio \& Minelli, 2011. En 2011 , le nombre de taxons valides et formellement connus au niveau départemental s'élevait à 68 (Iorio 2008, 2010a; Bonato et al. 2011).

Les chilopodes des Alpes-Maritimes et de leurs environs apparaissent comme relativement bien connus actuellement. Il est vrai que le littoral de Cannes à Menton de même que d'autres sites à l'Est du fleuve Var, tels que les vallées et/ou reliefs de l'arrière-pays niçois et cannois, de la Bévéra, et plus encore de la Vésubie, de la Madonne de Fenestre et du Boréon, disposent aujourd'hui d'assez nombreuses données publiées, surtout au regard d'autres secteurs délaissés dans le Sud-Est de la France. Néanmoins, de vastes massifs et vallées restent presque complètement inexplorés, comme la majeure partie des Préalpes de Grasse (Estéron, Cheiron, Audibergue), la partie amont du Var, et la majorité de l'Ouest du département au nord du fleuve.

Ces dernières années (2008 à 2013), nous avons récolté et identifié de nombreux chilopodes issus de diverses localités de l'aire concernée, provenant de milieux épigés comme de milieux souterrains, en partie dans le cadre de l'ATBI (All Taxa Biodiversity Inventories) du Parc national du Mercantour. Une révision de spécimens concernant une espèce à confirmer en
France a également été effectuée. Les données nouvellement acquises complètent les précédentes et permettent notamment la description d'une espèce nouvelle pour la science, mais aussi des constats de disparition potentielle au sein de localités historiques pour plusieurs géophilomorphes aux exigences écologiques particulières. Le présent travail a aussi pour objectif de dresser un premier inventaire des chilopodes du Parc national du Mercantour, de mettre à jour la liste des espèces du département des Alpes-Maritimes, ainsi que de fournir une première synthèse sur l'intérêt biogéographique de ces secteurs concernant les chilopodes, en soulignant la répartition très limitée et/ou la sensibilité écologique notable de certains taxa. Nous espérons ainsi mettre en évidence la nécessité de préservation pour plusieurs d'entre eux, au regard de l'anthropisation croissante et déjà importante de ce département.

\section{MATÉRIEL ET MÉTHODES}

De 2008 à 2013, de nombreuses espèces ont fait l'objet de collectes en diverses localités du département des AlpesMaritimes et de ses environs, notamment dans et à proximité de la zone cour et de la zone d'adhésion du Parc national du Mercantour dans le cadre de l'ATBI mené sur le PNM. En outre, des collègues zoologistes (François Breton, Grégory Déso, Jean-Michel Lemaire, Jean Raffaldi, Marielle Tardy et Augusto Vigna Taglianti) ont récolté des chilopodes et nous les ont confiés afin que nous puissions les étudier et les mettre en collection. Les secteurs peu explorés jusqu'alors ont été privilégiés, mais des données ont aussi été acquises dans des localités déjà prospectées, permettant de compléter les connaissances antérieures. De nombreux types d'habitats ont été inspectés, quelques-uns plus particuliers faisant l'objet de recherches soutenues; c'est le cas des cavités (grottes, avens, profondes fissures). La plupart des spécimens a été récoltée à vue. Dans chaque milieu naturel ou artificiel, les repaires favorables à ces arthropodes pour la plupart hygrophiles, lucifuges et sujets au thigmotropisme ont été minutieusement examinés: les pierres, les rondins, les écorces au sol ou sur des arbres morts et les mousses sur des rochers ont été retournés durant la journée. Les spécimens repérés ont été immédiatement placés en flacons dans l'éthanol $70 \%$, où ils ont été fixés et conservés en attendant de les étudier. Les individus provenant de cavités souterraines ont été capturés à l'aide de pièges terrestres contenant un liquide attractif et conservateur (canettes de bière emplies au quart de vin aromatisé et stabilisé), laissés sur place durant plusieurs semaines (voire mois), puis récupérés pour en extraire les individus capturés qui sont alors fixés dans l'éthanol (Jean-Michel Lemaire et Jean Raffaldi coll.).

Dans les Alpes-Maritimes, des Geophilomorpha vivant dans la zone intertidale et celle, adjacente, mouillée par les vagues, ont été signalées à plusieurs reprises par d'anciens auteurs (Caziot 1925; Verhoeff 1928; Attems 1929; Brölemann 1930): Geophilus fucorum Brölemann, 1909 et Tuoba poseidonis (Verhoeff, 1901) (Geophilidae), strictement inféodés 
à cet habitat; Henia (Meinertia) bicarinata (Meinert, 1870) (Dignathodontidae) et Pachymerium ferrugineum (C. L. Koch, 1935) (Geophilidae), qui privilégient ce milieu. En vue de remettre à jour leur connaissance locale, l'un d'entre nous (EI) a mené, à l'aide d'échantillonnages directs, des recherches ciblées sur ces espèces dans les stations citées par ces auteurs et d'autres inédites. La liste des localités prospectées est la suivante (avec précision du ou des micro-habitat(s) ciblé(s), de la période d'échantillonnage et, pour les localités historiques, des espèces citées et des auteurs).

\section{STATIONS HISTORIQUES}

Antibes (06)

Cap d'Antibes, entre la Batterie du Graillon et la Pointe de l'Îlette, bord de mer au niveau de la zone intertidale, de la zone de mouillage par les vagues et un peu en amont de celle-ci, sous les pierres et les débris végétaux échoués, septembre 2009 (H. bicarinata, P. ferrugineum: Caziot 1925; Brölemann 1930).

\section{Cannes (06)}

À proximité de la Croisette, plage Gazagniaire, plage de la Croisette, au niveau de la zone intertidale, de la zone de mouillage par les vagues et un peu en amont de celle-ci, sous les pierres, juin 2008, septembre 2008, juin 2009, octobre 2009 (G. fucorum, P. ferrugineum: Caziot 1925; Brölemann 1930).

\section{Cannes (06)}

Cannes-la-Bocca, plage de la Bocca et Rochers de la Bocca, au niveau de la zone intertidale, de la zone de mouillage par les vagues et un peu en amont de celle-ci, sur la paroi rocheuse et les rochers dans les fissures, sous les pierres, dans les rares trous envasés, juin 2008, septembre 2008, juin 2009, octobre 2009 (G. fucorum, P. ferrugineum: Caziot 1925; Brölemann 1930).

\section{Saint-Raphaël (83)}

À l'est de la plage du Veil et du côté de Santa Lucia et de la plage des Fournas, sous les pierres, sur les rochers et sous les rares débris végétaux, juin 2010 (G. fucorum: Verhoeff 1928; Attems 1929).

À noter que P. ferrugineum et de façon moindre G. fucorum étaient même considérés comme "présent sur tout le littoral " des Alpes-Maritimes par Caziot (1925) et Brölemann (1930).

\section{STATIONS INÉDITES}

Agay (83)

Plage du Pourrousset, bord de mer au niveau de la zone intertidale, de la zone de mouillage par les vagues et un peu en amont de celle-ci, sous les pierres et les rares débris végétaux échoués, juin 2010 .

\section{Ėze-Bord-de-Mer (06)}

Plage, bord de mer au niveau de la zone intertidale, de la zone de mouillage par les vagues et un peu en amont de celle-ci, sous les pierres et les rares débris végétaux échoués, juin 2007.
Nice (06)

Rauba Capeu et environs, bord de mer, parois rocheuses, faces inférieures des pierres sur la plage, fissures, etc. au niveau de la zone intertidale et de la zone de mouillage par les vagues, mai et juin 2006, juin 2007, septembre 2009.

\section{Saint-Jean-Cap-Ferrat (06)}

Pointe de la Cuisse, Pointe Malalongue, Pointe Causinière, Pointe du Colombier, Pointe de Saint-Hospice, Anse de la Scaletta, bords de mer en contrebas du Chemin de la Carrière et du Sentier du Bord de Mer, parois rocheuses, faces inférieures des pierres, fissures, halophytes, tas de posidonies échouées dans des creux de rochers mais aussi sous forme de laisses de mer sur des petites plages, trous envasés, sous les algues, etc. au niveau de la zone intertidale, de la zone de mouillage par les vagues et de flaques salées temporaires, voire juste en arrière de ces zones sur les plages, septembre 2008, juin 2009, septembre et octobre 2009, avril 2010.

\section{Villeneuve-Loubet (06)}

Plage près de Marina Baie des Anges, bord de mer, septembre 2006, juin 2008.

La grande majorité des chilopodes ne peut être identifiée sur le terrain et a nécessité un examen morphologique rigoureux sous loupe binoculaire, voire des préparations temporaires pour certaines parties à observer sous microscope. On se rapportera aux méthodes d'observations des chilopodes dans les ouvrages de Brölemann (1930) et Iorio (2006, 2010b).

Nos examens morphologiques s'appuient sur les travaux d'Attems (1929, 1947), Brölemann (1930), Koren (1986), Iorio (2006, 2008, 2010a, b), Iorio \& Geoffroy (2008) et Bonato et al. (2011) pour identifier l'ensemble des espèces capturées.

La taxonomie utilisée pour l'établissement de la liste des espèces récoltées, tout comme le Tableau 1 incluant la liste des chilopodes recensés dans le Parc national du Mercantour et la liste actualisée des espèces du département des Alpes-Maritimes, s'appuie essentiellement sur Geoffroy \& Iorio (2009), Iorio (2010b, 2014), Stoev et al. (2010) et Minelli et al. (2013).

Pour chaque taxon, la localisation réunit au minimum les informations suivantes: commune, (numéro du département), éventuelle provenance du Parc national du Mercantour, lieu-dit le plus proche ou localité topographique (nom de vallée, de vallon, de sommet, de hameau...), récolteur et déterminateur. Des commentaires sont inclus sur la répartition, sur l'écologie et parfois sur la morphologie. Le Tableau 1 reporte toutes les espèces et sous-espèces formellement connues et valides : dans l'ensemble zone d'adhésion/cœur de parc du Parc national du Mercantour; dans le département des Alpes-Maritimes. Les espèces uniquement cochées dans la colonne correspondant au Mercantour proviennent de la partie Est des Alpes-de-HauteProvence. Les espèces présentes dans les communes limitrophes de la première et/ou de la deuxième aire sont aussi indiquées. Ce Tableau inclut également, entre autres, les informations suivantes selon les cas: endémisme local (espèces endémiques des Alpes maritimes au sens de la SOIUSA (Marazzi 2005), voire des Alpes maritimes et/ou de leurs abords immédiats: Sud des Alpes cottiennes, Préalpes de Nice, Est des Préalpes de 
TABLEAU 1. - Liste des taxa de la classe Chilopoda formellement connus dans le Parc national du Mercantour (PNM, cœur du Parc + zone d'adhésion) et/ou dans le département des Alpes-Maritimes (A-M), d'après la méthode décrite et lorio (2008, 2010a, 2010b), Billi et al. (2011), Bonato et al. (2011) et le présent travail. Abbréviations : End. loc.: endémique local; End. nat.:endémique national; la mention de " $\mathbf{r}$ »: régression est en rapport avec les recherches ciblées relatées pour les halobiontes et les halophiles; (x): espèce mentionnée dans une commune limitrophe de l'enceinte du Parc et/ou du département des Alpes-Maritimes.

\begin{tabular}{|c|c|c|c|c|c|c|}
\hline Espèce & PNM & A-M & End. loc. End. nat. & $\begin{array}{l}\text { Présence en } \\
\text { limite d'aire }\end{array}$ & $\begin{array}{c}\text { Écologie } \\
\text { particulière }\end{array}$ & $\begin{array}{l}\text { Répartition } \\
\text { altitudinale } \\
\text { connue (en m) }\end{array}$ \\
\hline Scutigera coleoptrata (Linnaeus, 1758) & & $x$ & & & & $0-650$ \\
\hline Lamyctes emarginatus (Newport, 1844) & $x$ & $x$ & & & & 1730 \\
\hline Eupolybothrus grossipes (C. L. Koch, 1847) & & $x$ & & Ouest & & 200 \\
\hline Eupolybothrus longicornis (Risso, 1826) & $x$ & $x$ & & & & $150-2450$ \\
\hline Eupolybothrus nudicornis (Gervais, 1837 & $x$ & $x$ & & & & $0-2100$ \\
\hline HarpoLithobius anodus (Latzel, 1880) & & $x$ & & Ouest & & 143 \\
\hline Lithobius acuminatus Brölemann, 1892 & $x$ & $x$ & & Ouest & & 1500 \\
\hline Lithobius agilis C. L. Koch, 1847 & & $x$ & & & & $143-800$ \\
\hline Lithobius calcaratus C. L. Koch, 1844 & $x$ & $x$ & & & & $16-1600$ \\
\hline Lithobius castaneus Newport, 1844 & $x$ & $x$ & & & & $600-800$ \\
\hline Lithobius delfossei lorio \& Geoffroy, 2007 & $(x)$ & & $x$ & Est & Monticole & $1100<$ alt. \\
\hline Lithobius dentatus C. L. Koch, 1844 & $\times$ & $x$ & & Sud & & $1350-1450$ \\
\hline Lithobius erythrocephalus C. L. Koch, 1847 & & $x$ & & & & $0-700$ \\
\hline Lithobius forficatus Linnaeus, 1758 & & $x$ & & & & $0-?$ \\
\hline Lithobius lapidicola Meinert, 1872 & $x$ & $\times$ & & & & $60-2400$ \\
\hline Lithobius lemairei $\mathrm{n} . \mathrm{sp}$. & & $(x)$ & $x$ & & Troglobie & $900-1000$ \\
\hline Lithobius Iucifugus L. Koch, 1862 & $x$ & $\times$ & & & Monticole & $1000-1830$ \\
\hline Lithobius melanops Newport, 1845 & & $x$ & & & & 860 \\
\hline Lithobius mutabilis L. Koch, 1862 & $x$ & $x$ & & & & $0-1500$ \\
\hline Lithobius nicoeensis Brölemann, 1904 & & $x$ & & Ouest & & $0-800$ \\
\hline Lithobius piceus verhoeffi Demange, 1958 & $x$ & $x$ & & & & $400-1437$ \\
\hline Lithobius pilicornis Newport, 1844 & $x$ & $x$ & & & & $16-2750$ \\
\hline Lithobius pyrenaicus Meinert, 1872 & & $x$ & & Est & & $0-900$ \\
\hline Lithobius salicis Verhoeff, 1925 & & $x$ & & Ouest & & alt. $<2136$ \\
\hline Lithobius scotophilus Latzel, 1887 & $x$ & $x$ & $x$ & Ouest & Troglobie & $900<$ alt. \\
\hline Lithobius tricuspis Meinert, 1872 & $x$ & $x$ & & & & $140-3035$ \\
\hline Lithobius valesiacus Verhoeff, 1935 & $x$ & $x$ & & & Monticole & $1320-2961$ \\
\hline Lithobius validus Meinert, 1872 & & $x$ & & & & $550-600$ \\
\hline Lithobius micropodus (Matic, 1980) & & $x$ & & & & $0-600$ \\
\hline Lithobius microps Meinert, 1868 & $x$ & $x$ & & & & $0-2450$ \\
\hline Scolopendra cingulata Latreille, 1829 & & $x$ & & & & $0-300$ \\
\hline Cryptops anomalans Newport, 1844 & & $x$ & & & & $0-?$ \\
\hline Cryptops hortensis (Donovan, 1810) & $x$ & $x$ & & & & $0-1600$ \\
\hline Cryptops lobatus Verhoeff, 1931 & $x$ & $x$ & & Nord & & $240-1380$ \\
\hline Cryptops parisi Brölemann, 1920 & $x$ & $x$ & & & & $143-2400$ \\
\hline Cryptops trisulcatus Brölemann, 1902 & $x$ & $x$ & & & & $0-1500$ \\
\hline Cryptops umbricus umbricus Verhoeff, 1931 & & $\times$ & & Ouest & Eutroglophile & $0-1000$ \\
\hline Cryptops umbricus lewisi lorio, 2010 & $x$ & $x$ & $x$ & & Eutroglophile & $910-1400$ \\
\hline Himantarium gabrielis (Linnaeus, 1767) & $\times$ & $x$ & & & & $0-1050$ \\
\hline Stigmatogaster arcisherculis (Brölemann, 1904) & & $x$ & $x$ & Ouest & & $0-600$ \\
\hline Stigmatogaster dimidiata (Meinert, 1870) & & $\times$ & & & & $400-600$ \\
\hline Stigmatogaster gracilis (Meinert, 1870) & & $x$ & & & & $0-1000$ \\
\hline Dignathodon microcephalus Lucas, 1846 & $x$ & $x$ & & & & $0-1600$ \\
\hline Henia vesuviana (Newport, 1844) & $x$ & $x$ & & & & $0-2450$ \\
\hline Henia bicarinata (Meinert, 1870) & & $r$ & & & Halophile & 0 \\
\hline Henia brevis (Silvestri, 1896) & $x$ & $x$ & & & & $0-800$ \\
\hline Schendyla cf. apenninorum (Brölemann \& Ribaut, 1911) & & $x$ & & (Nord-ouest) & & $1320-1450$ \\
\hline Schendyla armata Brölemann, 1901 & & $x$ & & Ouest & & $0-350$ \\
\hline Schendyla carniolensis Verhoeff, 1902 & & $x$ & & & & $0-1400$ \\
\hline Schendyla mediterranea Silvestri, 1897 & & $x$ & & & & $0-1050$ \\
\hline Schendyla monoeci Brölemann, 1904 & & $x$ & & Ouest & & $0-?$ \\
\hline Schendyla montana (Attems, 1895) & $x$ & $x$ & & & Monticole & $1600-1700$ \\
\hline Schendyla nemorensis (C. L. Koch, 1837) & & $\times$ & & & & $0-600$ \\
\hline Clinopodes vesubiensis Bonato, lorio \& Minelli, 2011 & $x$ & $x$ & $x$ & & Monticole & $1130-2150$ \\
\hline Geophilus alpinus Meinert, 1870 & $\times$ & $\times$ & & & & $0-1500$ \\
\hline Geophilus bobolianus Verhoeff, 1928 & & $x$ & & Est & & $0-?$ \\
\hline Geophilus carpophagus Leach, 1815 s. I. & $x$ & $x$ & & & & $0-2000$ \\
\hline Geophilus electricus (Linnaeus, 1758) & & $x$ & & Sud & & $1320-1450$ \\
\hline Geophilus flavus (De Geer, 1778) & $x$ & $x$ & & & & $0-2450$ \\
\hline Geophilus fucorum Brölemann, 1909 & & $r$ & & & Halobie & 0 \\
\hline Geophilus joyeuxi Léger \& Duboscq, 1903 & & $x$ & $x$ & Est & & $0-?$ \\
\hline Geophilus richardi Brölemann, 1904 & & $x$ & & Ouest & & $0-600$ \\
\hline Geophilus truncorum ribauti Brölemann, 1908 & $x$ & $x$ & & Est & Monticole & $1050-2300$ \\
\hline Pachymerium ferrugineum (C. L. Koch, 1835) & & $r$ & & & Halophile & 0 \\
\hline Pleurogeophilus mediterraneus (Meinert, 1870) & $\times$ & $\times$ & & Est & & $40-1200$ \\
\hline
\end{tabular}




\begin{tabular}{|c|c|c|c|c|c|c|}
\hline Espèce & PNM & A-M & End. loc. End. nat. & $\begin{array}{l}\text { Présence en } \\
\text { limite d'aire }\end{array}$ & $\begin{array}{c}\text { Écologie } \\
\text { particulière }\end{array}$ & $\begin{array}{l}\text { Répartition } \\
\text { altitudinale } \\
\text { connue (en m) }\end{array}$ \\
\hline Stenotaenia linearis (C. L. Koch, 1835) & $\times$ & $x$ & & & & $0-1450$ \\
\hline Tuoba poseidonis (Verhoeff, 1901) & & $r$ & & & Halobie & 0 \\
\hline Strigamia acuminata (Leach, 1915) & $x$ & $x$ & & & & $1350-2000$ \\
\hline Strigamia cottiana (Verhoeff, 1935) & $x$ & $x$ & $x$ & Sud & Monticole & 1950 \\
\hline Strigamia crassipes (C. L. Koch, 1835) & $x$ & $x$ & & & & $550-2400$ \\
\hline Strigamia transsilvanica (Verhoeff, 1928) & $x$ & $x$ & & & & $1000-2300$ \\
\hline
\end{tabular}

Castellane, Ouest des Alpes ligures) et/ou endémisme national (espèces endémiques de France). Les espèces endémiques locales non reprises au niveau de l'endémisme national sont donc logiquement des espèces sub-endémiques à ce niveau (= présentes en France et en Italie).

\begin{tabular}{|c|c|}
\hline \multirow{2}{*}{\multicolumn{2}{|c|}{$\begin{array}{l}\text { ABRÉVIATIONS } \\
\text { Récolteurs/déterminateurs }\end{array}$}} \\
\hline & \\
\hline & récolteur; \\
\hline $\begin{array}{l}\text { dét. } \\
\text { AVT }\end{array}$ & $\begin{array}{l}\text { déterminateur; } \\
\text { Augusto VIGNA TAGLIANTI; }\end{array}$ \\
\hline EI & Étienne IORIO; \\
\hline FB & François BRETON; \\
\hline HWB & Henri-Wilfried BROLEMANN; \\
\hline GD & Grégory DESO; \\
\hline JJG & Jean-Jacques GEOFFROY; \\
\hline JML & Jean-Michel LEMAIRE; \\
\hline JR & Jean RAFFALDI; \\
\hline PF & Pierre FRAPA; \\
\hline SB & Stéphane BENCE; \\
\hline SS & Sandrine SALMON; \\
\hline MT & Marielle TARDY; \\
\hline & Marzio ZAPPAROLI. \\
\hline
\end{tabular}

\section{Spécimens/morphologie}

a épine antérieure;

D dorsal(e);

$\mathrm{DaH} \quad$ épine dorso-antérieure de la hanche;

$\mathrm{DpF} \quad$ épine dorso-postérieure du fémur :

ex. $\quad$ exemplaire(s);

Fé fémur;

$\mathrm{H}$ hanche;

m épine médiane;

$\mathrm{p} \quad$ épine postérieure;

Pf préfémur;

pp paires de pattes (Geophilomorpha);

P15 patte(s) 15 (ou quinzième paire de pattes);

T tibia;

T9 tergite 9 (ou tergite du 9e segment pédifere);

tr trochanter;

$\mathrm{V} \quad \operatorname{ventral}(\mathrm{e})$

$\mathrm{VaF} \quad$ épine ventro-antérieure du fémur;

$\mathrm{VaH}$ épine ventro-antérieure de la hanche (= épine coxola térale);

$\mathrm{VpF} \quad$ épine ventro-postérieure du fémur.

\section{Institutions}

MNHN Muséum national d'Histoire naturelle, Paris;

PNM Parc national du Mercantour (dans son ensemble: cœur de Parc + zone d'adhésion).

Divers

MSS

Milieu souterrain superficiel.

\section{SYSTÉMATIQUE}

Ordre SCUTIGEROMORPHA Pocock, 1895

Famille SCUTIGERIDAE Leach, 1814

Genre Scutigera Lamarck, 1801

Scutigera coleoptrata (Linnaeus, 1758)

Scolopendra coleoptrata Linnaeus, 1758: 638.

LOCALITÉ TYPE. - Espagne.

MATÉRIEL EXAMINÉ. - Mandelieu (06), garrigue, en lisière arborée (chênes), 16 m, 43,528558N, 6,914716E, 7.V.2010, réc./dét. EI : 1 ex.; Valbonne (06), Sophia-Antipolis, pins, chênes verts et chênes pubescents, clairières pierreuses, $218 \mathrm{~m}, 43,625211 \mathrm{~N}, 7,03265 \mathrm{E}$, 11.IV.2012, réc./dét. EI : 2 ex.; Roquebrune-Cap-Martin (06), hôtel Vista Palace, jardins en restanques et pinèdes, $208 \mathrm{~m}, 43,756402 \mathrm{~N}$, 7,442974E, 18.IV.2012 et 26.VI.2012, réc./dét. EI: 3 ex.; Beausoleil (06), Giram, chênes verts, 499 m, 43,758534N, 7,416478E, 23.IV.2012, réc./dét. EI: 4 ex.; Nice (06), Pointe Madame, lisières de friches/pinèdes, 40-50 m, 3.X.2012, 43,692375N, 7,307853E, réc./dét. EI : 2 ex.; idem, 13.V.2013: 1 ex.; Menton (06), à proximité de la Gardieura, sentier GR 52, pinèdes et lisières de pinèdes, 350400 m, 3.V.2013, réc./dét. EI: 2 ex.; Roquebrune-Cap-Martin (06), Mont Gros, pinède claire, 600-650 m, 12.VI.2013, réc./dét. EI: 2 ex.; Menton (06), à proximité de Moniéri, mur le long de la petite route et de la lisière arbustive/arborée adjacente et près de quelques habitations en contrebas, 250-300 m, 9.X.2013 et 23.X.2013, réc./dét. EI : 9 ex.; Menton (06), Chemin des Granges de Saint-Paul, dans une lisière pierreuse de vignes, dans une friche, une prairie et une pinède, 400450 m, 24.X.2013, réc./dét. EI : 4 ex. Les spécimens ont été identifiés à vue pour cette espèce, très aisée à reconnaître sans prélèvement.

RÉPARTITION ET HABITAT. - Cette espèce largement répartie en France et en Europe mais quasi-exclusivement synanthrope en dehors des régions circumméditerranéennes, dont la distribution s'est sans aucun doute élargie en raison de ses mœurs anthropophiles, est ici dans son territoire d'autochtonie (Iorio \& Geoffroy 2007b). Dans les Alpes-Maritimes, on la trouve aussi bien en milieu naturel ou anthropisé qu'en milieu urbain et sub-urbain (Iorio 2008; présent travail), de 0 à 650 mètres d'altitude.

Ordre LITHOBIOMORPHA Pocock, 1895

Famille LITHOBIIDAE Newport, 1844

Genre Eupolybothrus Verhoeff, 1907

Eupolybothrus longicornis (Risso, 1826)

Lithobius longicornis Risso, 1826: 153.

Eupolybothrus fasciatus - Zapparoli 1980: 282, non Eupolybothrus fasciatus (Newport, 1844). 
LOCALITÉ TYPE. — France, Raus.

MatéRIEl EXAminé. - Beuil (06), PNM, Les Launes, conifères et lisière pelouse, $1600 \mathrm{~m}, 7 . V I .2008$, réc./dét. EI: $20^{\text {7 }} 2$ \% ; Guillaumes (06), PNM, Le Coulet, coniferes divers, $1470-1500$ m, 8.VI.2008, réc./dét. EI : $10^{7}, 1$ \% ; Faucon-de-Barcelonnette (04), Les Granges, landes et broussailles, $1661 \mathrm{~m}, 44,41139 \mathrm{~N}, 6,673783 \mathrm{E}, 9 . X I .2008$, réc./dét. FB : 19 ; Entrevaux (04), à proximité de Combe Guénier, 900-1000 m d'altitude, boisement de coniferes et de hêtres, VII.2009, réc./dét. EI : $10^{7}$; Saint-Dalmas-le-Selvage (06), PNM, La Buisse, lisière d'un bois, en surface, $1437 \mathrm{~m}, 44.2848357 \mathrm{~N}$, 6.8875257E, 17.VIII.2009, réc./dét. JJG : 1 \% immature; Péone (06), PNM, Gîte d'Aliège, prairies et garrigues boisées, friches, 1400 m, 18.VIII.2009, réc./dét. JJG: $10^{7}$; Allos (04), PNM, Lac

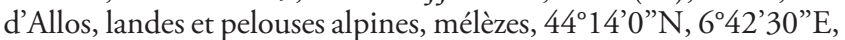
2230 m, 23.VIII.2009, réc./dét. JJG : $10^{\circ}$, 1 ㅇ ; Saint-MartinVésubie (06), PNM, vallon Madone de Fenestre, adret, $1200 \mathrm{~m}$, $44^{\circ} 04^{\prime} 57^{\prime \prime} \mathrm{N}-7^{\circ} 16^{\prime} 36^{\prime \prime E}, 22 . V .2010$, réc. AVT/dét. MZ: $20^{`}$; SaintMartin-Vésubie (06), PNM, Forêt de Boréon, parking 5, forêt mixte de coniferes, $1500 \mathrm{~m}, 44^{\circ} 06^{\prime} 59^{\prime \prime} \mathrm{N}-7^{\circ} 16^{\prime} 44^{\prime} \mathrm{E}, 21 . \mathrm{V} .2010$, réc./dét. MZ: $10^{7}$; Saint-Martin-Vésubie (06), PNM, vallon Madone de Fenestre, ubac, $1600 \mathrm{~m}, 44^{\circ} 05^{\prime} 25^{\prime \prime} \mathrm{N}-7^{\circ} 19^{\prime} 53^{\prime \prime E}, 22$.V.2010, réc./ dét. MZ: $20^{7}$; Saint-Martin-Vésubie (06), PNM, vallon Madone de Fenestre, ubac, sapinière-pessière, près d'une petite rivière,

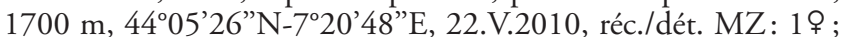
Saint-Dalmas-le-Selvage (06), PNM, près du Col de Restefond, $2400 \mathrm{~m}, 4^{\circ} 20^{\prime} 15.9^{\prime \prime} \mathrm{N}, 006^{\circ} 47^{\prime} 13.3^{\prime \prime} \mathrm{E}, 3 . \mathrm{VII} .2010$, réc. AVT/ dét. MZ: $10^{\prime \prime}, 2$ \% ; Allos (04), PNM, près du Lac d'Allos, 2140 m,

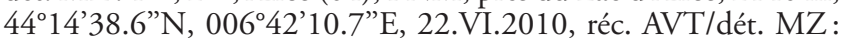
1 \% ; Tende (06), Marguareis, grotte, 20.IX.2010, réc. JR/dét. EI: $2 \%$; Tende (06), PNM, Granile, grotte de Fouige, 2010-2011, réc. JML/dét. EI: 19 ; Calern (06), aven du Cerisier, V-XI.2011, réc. JML/dét. EI : 5ㅇ ; idem, autre piège 2011: $20^{7}$, 4 우 ; Calern (06), aven de la Rougne, 2011, réc. JML/dét. EI : $10^{7}, 3$ \% ; Peille (06), Mont Agel, 15.XI.2011, réc. JML/dét. EI : 1\% ; Roubion (06), PNM, petite grotte non nommée, réc. JML/dét. EI : 10 , 19 ; Calern (06), grotte, 2011, réc. JML/dét. EI: 1 \% ; Roubion (06), Vignols, PNM, massif du Mounier, aven de Granréon, 2011, réc. JML/dét. EI : 1 \% ; Belvédère (06), PNM, vers Tres Crous, sentier à $1700 \mathrm{~m}$, réc. GD/dét. EI : $10^{7}$; Peille (06), grotte de Morgelle (dite de "Saint-Jean »), 2012, réc. JML/dét. EI : $20^{\text {* }}$; Peille (06), aven de Faille, 816 m, 5.IX.2013, réc. JML/dét. EI : 1 \% ; Aspremont/ Castagniers (06), vallon du Donaréo, dans le vallon obscur sous une pierre, 21.XI.2013, réc./dét. EI : $20^{7}$. Spécimens conservés en éthanol à $70^{\circ}$ dans la collection personnelle des auteurs (EI, MZ) ou dans celle du MNHN (Myriapoda, Lithobiomorpha) (JJG).

RÉpARTition eT habitat. - E. longicornis, présent dans le Nord de l'Italie et dans le Sud de la France, est l'un des lithobiomorphes les plus fréquents dans les Alpes-Maritimes (Iorio 2008; présent travail), où on le trouve de 150 à 2450 mètres d'altitude. En France, l'espèce vit essentiellement dans le Sud-Est; sa répartition s'étend jusqu'en Isère et en Savoie au nord, et jusque dans le Lot à l'ouest (Iorio 2014). 66 des stations connues dans le département des Alpes-Maritimes renseignent sur l'habitat où ont été observés les spécimens (Iorio 2008, 2010b; présent travail) : 25 sont des forêts de conifères (neuf pinèdes, six pessières, five sapinières-pessières, une sapinière, une lariçaie, trois boisements de conifères divers), deux des forêts mixtes (une pinède-sapinière-hêtraie, une conifères divers-hêtres), dix des forêts de feuillus (sept châtaigneraies, une châtaigneraie-corylaie, une ripisylve, une feuillus divers) et quatre des forêts indéterminées et/ou leurs lisières. Les milieux ouverts et semi-ouverts peuvent aussi être occupés (deux prairies alpines, deux prairies sub-alpines, deux prairies à rhododendrons, une prairie/friche montagnarde, une lande broussailleuse, une près d'un lac, une près d'un sentier), de même que les vallons obscurs de l'arrière-pays niçois (deux stations) et souvent les grottes (généralement, à l'entrée et/ou à proximité) (13 stations). Ainsi, l'espèce est relativement euryèce tout en privilégiant les milieux forestiers, et une certaine humidité. Cela correspond aux constats sur son ubiquité émis dans les régions adjacentes (Zapparoli 1993).

\section{REMARQUES}

Les deux mâles de la grotte de Morgelle possèdent des antennes et pattes plus longues qu'à l'accoutumée chez cette espèce dotée de long appendices. Premier mâle: taille: $23 \mathrm{~mm}$; longueur des antennes : 21,2 $\mathrm{mm}$; longueur des P15: 18,3 mm. Deuxième mâle: taille: $22 \mathrm{~mm}$; longueur des antennes: inconnue (en partie sectionnées); longueur des P15 : 20,2 mm. Les rapports de longueur des articles par rapport à la tête et de certains articles entre eux selon Brölemann (1930) sont ici dépassées; pour les individus ci-dessus, elles sont (tête $=100 \%$ ) : fémur/ tête: 135 à $138 \%$; tibia/tête: 169 à $176 \%$; basitarse ("tarse 1" de Brölemann)/tête: 179 à $180 \%$; basitarse/fémur (fémur = $100 \%$ ) : 130 à $134 \%$. Ils possèdent également des structures plus marquées aux P15 que la plupart des spécimens de la forme typique: en plus des sillons peu marqués qui s'observent aussi parfois chez le type sur certains articles, on distingue un renflement dorso-latéral interne plus développé sur le fémur des P15, égal à environ la moitié de la longueur de cet article. Ce renflement débute approximativement au milieu de l'article et se poursuit jusqu'à son extrémité, tandis qu'il est plutôt limité au tiers distal habituellement, et donc plus discret. En l'état actuel des connaissances, il paraît difficile d'attribuer une valeur discriminante à ces caractères, d'autant que certains individus d'E. longicornis pris en dehors de toute grotte présentent des dimensions proches des appendices antennaires et ambulatoires. Peut-être s'agit-il d'un début de spéciation de la population locale, liée à une vie exclusivement cavernicole, à l'instar de certains Lithobius (Lithobius) pilicornis Newport, 1844 ?

Zapparoli (1980) avait cité l'espèce "Eupolybothrus fasciatus (Newport, 1844)" dans les Alpes-Maritimes (Forêt de Turini), sur la base de cinq mâles et une femelle. Cette espèce de grande taille n'a jamais été retrouvée depuis, malgré les nombreux Eupolybothrus spp. capturés dans ce département. Au vu de l'absence de données récentes et du fait qu'elle soit localisée principalement dans le Centre et le Sud de l'Italie, les spécimens de Zapparoli (1980) en provenance de la Forêt de Turini ont été réexaminés: il s'agit en fait d'E. (E.) longicornis. $E$. fasciatus est donc à retirer de la faune française.

Eupolybothrus nudicornis (Gervais, 1837)

Lithobius nudicornis Gervais, 1837: 49.

LOCALITÉ TYPE. — Italie, Sicile.

MATÉRIEL EXAMINÉ. - Beausoleil (06), Giram, chênes verts, 499 m, 43,758534N, 7,416478E, 23.IV.2012, réc./dét. EI : 1 \% ; Mont Gros, flanc d'exposition nord-est, chênaie, 500-550 m, 30.X.2012, $43.7681 \mathrm{~N}, 7.44454 \mathrm{E}$, réc./dét. EI : $10^{7}$. Spécimens conservés en éthanol à $70^{\circ}$ dans la collection personnelle de l'auteur (EI).

RÉPARTITION ET HABITAT. - E. nudicornis est formellement présent en Italie continentale et insulaire, à Malte, dans le Sud-Est de la France, dans le Nord de l'Algérie, dans le Nord-Est du Maroc et 
en Tunisie (Zapparoli 2006; Stoev et al. 2010). En France, l'espèce est en limite d'aire occidentale et n'est présente qu'en ProvenceAlpes-Côte d'Azur (départements des Hautes-Alpes, des Alpes-deHaute-Provence et des Alpes-Maritimes) et en Corse (Iorio \& Berg 2007; Iorio 2010b). Bien que nettement moins commun que le précédent, E. nudicornis reste assez fréquent dans les Alpes-Maritimes, notamment dans les milieux encore plus ou moins naturels du littoral et des premiers reliefs de l'arrière-pays niçois et mentonnais. Nous disposons de moins d'informations sur les habitats que par rapport à E. longicornis: sur 11 stations où l'habitat est connu, sept sont des forêts (quatre sapinières-pessières, deux chênaies vertes, une pinède) et quatre des prairies sub-alpines et alpines. D'après Zapparoli (1993), E. nudicornis se trouve fréquemment dans les formations forestières thermophiles et méso-thermophiles, mais aussi parfois dans les milieux ouverts thermophiles. Sa répartition altitudinale dans les Alpes-Maritimes atteste tout de même d'une certaine tolérance vis-à-vis de facteurs abiotiques tels que fraîcheur et humidité, puisqu'il y est présent de 0 à 2100 mètres d'altitude.

\section{Genre Lithobius Leach, 1814}

\section{Lithobius (Lithobius) calcaratus}

C. L. Koch, 1844

\section{Lithobius calcaratus C. L. Koch, 1844: 23.}

LOCALITÉ TYPE. — Allemagne, Nuremberg.

MATÉRIEL eXAMiné. - Beuil (06), PNM, Les Launes, conifères et lisière pelouse, $1600 \mathrm{~m}, 7 . \mathrm{VI} .2008$, réc./dét. EI : 1 \% immature; Entrevaux (04), à proximité de Combe Guénier, 900-1000 m d'altitude, boisement de coniferes et de hêtres, VII.2009, réc./dét. EI : $30^{7}, 4$; ; Mandelieu (06), garrigue, en lisière arborée (chênes), $16 \mathrm{~m}, 43,528558 \mathrm{~N}, 6,914716 \mathrm{E}, 7 . V .2010$, réc./dét. EI : $10^{7}$; Valbonne (06), Sophia-Antipolis, friches/lisières de pinèdes, $150 \mathrm{~m}$, 43,638794N, 7,044013E, 10.IV.2012, réc./dét. EI : 1 ㅇ ; Valbonne (06), Sophia-Antipolis, pins, chênes verts et chênes pubescents, $236 \mathrm{~m}, 43,625116 \mathrm{~N}, 7,031673 \mathrm{E}, 11 . I V .2012$, réc./dét. EI : $20^{\prime}$; idem, 43,626743N, 7,036508E: 1 ㅇ ; Roquebrune-Cap-Martin (06), hôtel Vista Palace, jardins en restanques et pinèdes, $208 \mathrm{~m}$, 43,756083N, 7,443165E, 18.IV.2012, réc./dét. EI : $20^{7}, 2$; ; Beausoleil (06), Giram, garrigue et pelouses sèches, bosquet de chênes verts, $499 \mathrm{~m}, 43,754686 \mathrm{~N}, 7,418105 \mathrm{E}, 23 . I V .2012$, réc./dét. EI : 19; Digne-les-Bains (04), Le Serre, 1100 m, prairie humide, 27.V.2012, réc. PF/dét. EI : $10^{7}$ immature; Monaco, 12.I.2013, réc. JML/dét. EI : $10^{7}$; Nice (06), Pointe Madame, lisières de friches/ pinèdes, 40-50 m, 3.X.2012, 43,692375N, 7,307853E, réc./dét. EI: $10^{7}$; idem, 13.V.2013: 19; Roquebrune-Cap-Martin (06), Mont Gros, garrigues, bosquets de chênes verts et pelouses sèches, 500-650 m, 30.X.2012, réc./dét. EI : 307, 4우 ; Menton (06), à proximité de la Gardieura, sentier GR 52, pinèdes et lisières de pinèdes, 350-400 m, 3.V.2013, réc./dét. EI: 1 \% ; Menton (06), Baisse Saint-Paul, pelouses sèches pierreuses, 9.X.2013, réc./dét. EI : $10^{\top}$; Menton (06), à proximité du Chemin des Granges de Saint-Paul, dans des pelouses méso-xérophiles et dans une pinède, 400-450 m, 24.X.2013, réc./dét. EI : $50^{7}$ dont 1 immature, 5 o dont 1 immature. Spécimens conservés en éthanol à $70^{\circ}$ dans la collection personnelle de l'auteur (EI).

RÉPARTITION ET HABITAT. - - L. (L.) calcaratus est largement réparti en France et en Europe occidentale; il est connu dans tous les départements de la région Provence-Alpes-Côte d'Azur (Iorio \& Berg 2007; Iorio 2008, 2010b, 2014). 36 des stations connues dans le département des Alpes-Maritimes comportent des renseignements sur l'habitat des spécimens observés (Iorio 2008; présent travail) : 20 sont des milieux forestiers dont plusieurs thermophiles (sept pinèdes, une station de coniferes divers, deux stations de coniferes et hêtres, une chênaie-pinède, une chênaie-pinède claire en partie envahie par le " mimosa ", une chênaie-pinède avec chênes verts et pubescents, six chênaies dont trois chênaies vertes, une oliveraie), 15 sont des milieux ouverts xéro-thermophiles (sept garrigues, trois friches, trois pelouses sèches, un jardin en restanques); également cité d'une prairie humide. Il y est connu de 16 à 1600 mètres d'altitude. En dehors du département des Alpes-Maritimes, cette espèce est connue dans de nombreuses autres stations ouvertes mésophiles à xérophiles (Iorio 2007, 2010b, 2014; Iorio \& Berg 2007), et elle tolère bien les milieux relativement secs. En Italie centrale et septentrionale, elle est relativement thermophile et xérophile et privilégie les milieux ouverts, même anthropisés et dégradés tels que les agro-écosystèmes (Zapparoli 1993, 2006).

\section{Lithobius (Lithobius) dentatus}

C. L. Koch, 1844

\section{Lithobius dentatus C. L. Koch, 1844: 22.}

LOCALITÉ TYPE. - Allemagne, district de Nuremberg.

Matériel eXaminé. - Saint-Martin-Vésubie (06), PNM, Forêt de Boréon, parking 5, forêt mixte de conifères, $1500 \mathrm{~m}$, $44^{\circ} 06^{\prime} 59^{\prime}{ }^{\prime} \mathrm{N}-7^{\circ} 16^{\prime} 44^{\prime \prime} \mathrm{E}, 21 . V .2010$, réc./dét. MZ: 2 ㅇ. Spécimens conservés en éthanol à $70^{\circ}$ dans la collection personnelle de l'auteur $(\mathrm{MZ})$

RÉPARTITION ET HABITAT. - Cette espèce est essentiellement présente en Europe centrale et centro-occidentale; en France, elle vit dans les régions Haute-Normandie, Picardie, Champagne-Ardennes, Lorraine, Bourgogne, Rhône-Alpes et Provence-Alpes-Côte d'Azur (Iorio 2010b), où elle est globalement rare, probablement en raison du fait qu' elle atteint ici la limite orientale de sa répartition. Dans les Alpes-Maritimes, elle est présente dans seulement quatre stations sur plusieurs centaines explorées (cf. Iorio 2008, 2010b; présent travail). Les habitats des Alpes-Maritimes sont tous des forêts montagnardes: une pessière, une hêtraie-conifères, une lariçaie, une forêt mixte, situées entre 1350 et 1500 mètres d'altitude. Ceci correspond aux données du Nord de l'Italie (Minelli \& Iovane 1987): surtout des hêtraies et des hêtraies-sapinières montagnardes, mais aussi des forêts de coniferes montagnardes; 490 à 1500 mètres d'altitude. Dans le Nord-Est de la France et autres contrées, $L$. (L.) dentatus affectionne essentiellement les forêts caducifoliées planitiaires et collinéennes (Fründ et al. 1997; Spelda 1999; Lock et al. 2001; Iorio 2007; Kime \& Iorio 2010). Ainsi, cette espèce assez strictement sylvicole a une nette préférence pour les boisements de feuillus, préférence moins marquée dans le sud de sa répartition où, en contrepartie, elle est plus montagnarde.

\section{Lithobius (Lithobius) forficatus}

(Linnaeus, 1758)

Scolopendra forficata Linnaeus, 1758: 638.

\section{LOCALITÉ TYPE. — Europe.}

Matériel eXaminé. - Nice (06), Parc Phénix, 15.I.2012, réc. JML/ dét. EI : 19 ; idem, 8.I.2013: $10^{7}$. Spécimens conservés en éthanol à $70^{\circ}$ dans la collection personnelle de l'auteur (EI).

RÉPARTITION ET HABITAT. - Espèce euryèce largement répartie en France et en Europe, jusqu'au Moyen-Orient et introduite en Afrique du Nord et de nombreuses autres contrées; elle est globalement très commune (Zapparoli 2006; Iorio 2010b), bien que sporadique dans certains pays tels que l'Espagne (Serra 1980). Dans le département des Alpes-Maritimes, elle n'est bien répartie que sur littoral 
(Iorio 2008), mais est moins fréquente qu'ailleurs en France puisque quasiment aucune localité n'est connue dans l'arrière-pays malgré les nombreuses prospections réalisées en divers endroits (Iorio 2008, $2010 \mathrm{~b}$, présent travail). Elle y est très probablement surtout cantonnée aux milieux urbains et sub-urbains, et quasi-inexistante dans les milieux naturels où elle est concurrencée ou supplantée par $L$. (L.) pilicornis, qui y est très abondant (Iorio 2008). Ce phénomène de concurrence entre les deux espèces est déjà connu ailleurs, mais à altitude plus élevée (Geoffroy 1981b; Geoffroy \& Iorio 2009).

\section{Lithobius (Lithobius) lapidicola Meinert, 1872}

\section{Lithobius lapidicola Meinert, 1872: 328.}

LOCALITÉ TYPE. - Allemagne, Tubingen; Italie, Razzes, Campagne romaine, Sorrente; Espagne, Cordoba, Grenade; Algérie, Bone.

Matériel eXAminé. - Saint-Martin-Vésubie (06), PNM, sous le Col de Salèse, Rhododendron, sol, 1935 m, 44.1348693N, 7.2418197E, 10.VI.2009, réc. SS/dét. JJG: $10^{7} ;$ Monaco, grotte du château, 2010, réc. JML/dét. EI : $30^{7}, 3$; ; Saint-Martin-Vésubie (06), PNM, Forêt de Boréon, parking 5, forêt mixte de conifères, $1500 \mathrm{~m}$, $44^{\circ} 06^{\prime} 59^{\prime \prime} \mathrm{N}-7^{\circ} 16^{\prime} 44^{\prime} \mathrm{E}, 21 . V .2010$, réc. AVT/dét. MZ: $10^{\text {" }}$; Guillaumes (06), PNM, grotte Tremens, 2011, réc. JML/dét. EI : 1 ㅇ. Spécimens conservés en éthanol à $70^{\circ}$ dans la collection personnelle des auteurs (EI, MZ) ou dans celle du MNHN (Myriapoda, Lithobiomorpha) (JJG).

RÉPARTITION ET HABITAT. - L L ( L.) lapidicola est largement réparti en Europe occidentale et centrale. Il est essentiellement présent en France dans les régions montagneuses de l'Est (Vosges, Alpes dont Alpes maritimes) et de Corse, tandis que sa présence dans les Pyrénées est très douteuse (Iorio 2008, 2014; Geoffroy \& Iorio 2009). 14 des stations connues dans le département des Alpes-Maritimes fournissent des informations sur l'habitat où ont été observés les spécimens (Iorio 2008; présent travail) : huit prairies/pelouses subalpines et alpines, une lande à rhododendrons, une station au bord d'un lac de l'étage alpin, une mélézaie et une forêt mixte de conifères, et enfin deux grottes. Dans ce département, il est présent de 60 à 2400 mètres d'altitude. Comme évoqué précédemment (Iorio 2008), il semble affectionner surtout les pelouses alpines et subalpines dans des Alpes-Maritimes, tandis qu'il est plutôt euryèce en Italie (Minelli \& Iovane 1987; Zapparoli 1993). Dans d'autres départements du Massif alpin, les mêmes milieux apparaissent souvent (Iorio 2010b). On peut toutefois le trouver occasionnellement à basse altitude dans les grottes (Iorio 2010b; présent travail).

\section{Lithobius (Lithobius) lemairei n. sp.}

MATÉRIEL TYPE. - Holotype. Entrevaux (France, Alpes-de-HauteProvence), à proximité de Combe Guénier, 900-1000 m d'altitude, boisement de conifères et de hêtres, secteur rocheux à profondes fissures et éboulis, VII.2009, réc. EI/dét. EI \& MZ: 19. Collection MNHN, Myriapoda, Lithobiomorpha. Le bocal P270 comprend le spécimen avec l'ensemble de ses pattes à l'exception de la 9e paire; une des P14 et une des P15 se sont détachées du tronc lors de nos examens, mais y sont incluses.

LOCALITÉ TYPE. - Entrevaux (France, Alpes-de-Haute-Provence), à proximité de Combe Guénier, 900-1000 m d'altitude, boisement de conifères et de hêtres, secteur rocheux à profondes fissures et éboulis.

ÉTYMOLOGIE. - Nous dédions cette nouvelle espèce à notre collègue entomologiste Jean-Michel Lemaire, en remerciement de ses récoltes régulières et originales de chilopodes.
Diagnose. - Cette espèce du genre Lithobius se distingue par la combinaison des caractères suivants: sa petite taille $(8 \mathrm{~mm})$; la longueur modérée et la segmentation de ses antennes qui atteignent deux cinquièmes de la longueur du corps et comprennent 38 à 39 articles; la quasi-absence d'ocelles (une à deux ocelles très rudimentaires, peu visibles au microscope optique); la grande taille de l'organe de Tömösváry; le nombre de dents forcipulaires $(2+2)$ et l'aspect général de ses forcipules; l'absence de prolongements triangulaire aux tergites 9,11 et 13 ; le nombre de ses pores coxaux (deux par hanche); la disposition des épines de ses pattes, dont l'absence d'épine coxolatérale $(\mathrm{VaH})$; la longueur modérée des pattes de la dernière paire (P15); l'existence particulièrement notable d'un sillon longitudinal sur les faces latérales-postérieures des fémurs, tibias, basitarses et tarses des P14 et surtout des P15; la présence d'une griffe apicale simple aux P15; les gonopodes de la femelle qui possèdent $2+2$ éperons et une griffe tridentée. Le mâle demeure pour l'instant inconnu.

\section{DESCRIPTION DE L'HOLOTYPE}

Longueur du corps $8 \mathrm{~mm}$. La coloration générale est à dominante de fauve-jaune/orangé (Fig. 1A).

La longueur des antennes, qui comprennent $38+39$ articles, atteint $3,2 \mathrm{~mm}$, soit $2 / 5$ de celle du corps ; la longueur du dernier article atteint le triple de celle du pénultième. L'écusson céphalique ne possède qu'un fin bourrelet marginal au bord postérieur. Les ocelles sont extrêmement réduits (Fig. 2A) : à fort grossissement $(\times 50)$ et à éclairage intense, on distingue avec difficulté deux ocelles très rudimentaires, dépigmentés, du côté gauche de la tête, et un seul, similaire, du côté droit de la tête. L'organe de Tömösváry est très développé, beaucoup plus grand et visible que les ocelles. Le bord rostral du coxosternum forcipulaire est modérément proéminent et comprend $2+2$ dents forcipulaires et $1+1$ épines courtes et grêles de part et d'autre des dents externes (Figs 1B; 2B). Il n'y a aucune bosse latérale ni rebord anguleux de part et d'autre des dents externes, les bords latéraux étant assez régulièrement obliques jusqu'à la jonction avec le fémoroïde des forcipules.

Les angles postérieurs des tergites 9, 11 et 13 sont totalement dépourvus de prolongements triangulaires (Figs 1A; 2C). Ceux du tergite 9 sont droits, bien qu'un peu émoussés; il en est de même pour ceux des tergites 11 et 13 . Les autres tergites n'ont aucune particularité notable.

Les sternites des quatre derniers segments pédifères possèdent chacun quelques soies dispersées. Les hanches des quatre dernières paires de pattes possèdent aussi quelques soies dispersées. Elles sont dotées chacune de deux pores coxaux (PP.12-15: 2, 2, 2, 2), ronds, de taille moyenne (Fig. 1C). Il n'y a pas d'épine coxolatérale $(\mathrm{VaH})$ aux P.15.

La plectrotaxie, aussi nommée spinulation par Brölemann (1930), est présentée dans le Tableau 2.

Les fémurs, tibias, basitarses et tarses des P14 et surtout des P15 sont dotés, sur leur face latérale-postérieure (vue latérale-interne), d'un sillon longitudinal modérément prononcé mais tout de même bien visible (Figs 1C, D; 2D). Ce sillon est cependant plus marqué sur les trois articles distaux, notamment les tibias et les basitarses. Moins accentué sur les fémurs de ces pattes, notamment sur ceux des P14, il n'occupe que les $4 / 5$ de ceux-ci, voire moins. Il faut préciser que la P15 gauche, atrophiée, est très probablement un régénérat, encore 
TABlEAU 2. - Plectrotaxie des pattes de Lithobius (Lithobius) lemairei n. sp., holotype, femelle. Les P9 sont manquantes. L'épine VaT (a) de la P8 n'existe que d'un seul côté sur ce spécimen. Abréviations: voir Matériel et méthodes.

\begin{tabular}{|c|c|c|c|c|c|c|c|c|c|c|c|c|}
\hline & V: & H & tr & Pf & Fé & $\mathbf{T}$ & D : & H & tr & Pf & Fé & $\mathbf{T}$ \\
\hline P1 & & - & - & --- & $-m-$ & $-m-$ & & - & - & $--p$ & a-- & --- \\
\hline P2 & & - & - & --- & $-m-$ & $-m-$ & & - & - & $--p$ & $a-p$ & a-- \\
\hline P3 & & - & - & --- & $-\mathrm{m}-$ & $-m-$ & & - & - & --- & $a-p$ & $a-p$ \\
\hline P4 & & - & - & --- & am- & $-m-$ & & - & - & --- & $a-p$ & $a-p$ \\
\hline P5 & & - & - & --- & am- & $-m-$ & & - & - & --- & $a-p$ & $a-p$ \\
\hline P6 & & - & - & --- & am- & $-m-$ & & - & - & --- & $a-p$ & $a-p$ \\
\hline P7 & & - & - & --- & am- & -m- & & - & - & --- & $a-p$ & $a-p$ \\
\hline P8 & & - & - & --- & am- & (a)m- & & - & - & --- & $a-p$ & $a-p$ \\
\hline P9 & & - & - & $x$ & $x$ & $x$ & & - & - & $x$ & $x$ & $x$ \\
\hline P10 & & - & - & --- & am- & am- & & - & - & --- & $a-p$ & $a-p$ \\
\hline P11 & & - & - & --- & am- & am- & & - & - & --- & $a-p$ & $a-p$ \\
\hline P12 & & - & - & $-m p$ & amp & am- & & - & - & $--p$ & $--p$ & $a-p$ \\
\hline P13 & & - & $\mathrm{m}$ & $-m p$ & amp & am- & & - & - & $-m p$ & $--p$ & $--p$ \\
\hline P14 & & - & $\mathrm{m}$ & amp & $-m-$ & --- & & - & - & $-m p$ & --- & --- \\
\hline P15 & & - & $\mathrm{m}$ & $\mathrm{amp}$ & $-m-$ & --- & & a & - & $-m p$ & --- & --- \\
\hline
\end{tabular}

incomplet, d'une patte arrachée avant la dernière mue, ce qui explique que la structure décrite ci-dessus est donc moins évidente sur elle.

Sur ces mêmes faces latérales-postérieures, les P14 et surtout les P15 revêtent également de très nombreux pores minuscules. La griffe apicale des P15 est simple (pas de griffe annexe) et courte. La longueur totale de la P15 non atrophiée est de $2,9 \mathrm{~mm}$.

Les gonopodes sont dotés de deux éperons assez longs sur chaque article basal, un peu divergents l'un de l'autre bien que quasi-accolés à leur base (Figs 1C; 2E); ils sont acuminés dans les $3 / 5$ distaux de leur longueur, ce qui leur confère un aspect bien pointu. La griffe gonopodiale est d'aspect tridenté, la dentelure dorsale étant nettement plus forte que la ventrale; la dentelure dorsale est la plus proche de la pointe.

\section{COMPARAISONS AVEC LES ESPÈCES PROCHES}

DE LITHOBIUS (LITHOBIUS) LEMAIREI N. SP.

En ce qui concerne les chilopodes, les grottes des AlpesMaritimes ne font l'objet de récoltes régulières que depuis quelques années (Iorio 2010a, 2010b ; présent travail); une partie d'entre elles avaient été plus ou moins étudiées par les auteurs (Caziot 1925; Jeannel 1926; Manfredi 1932; Zapparoli 1980, 1993; Minelli \& Zapparoli 1985, 1992; Iorio 2008). Comme cela est illustré plus haut pour certaines espèces (Eupolybothrus longicornis, Lithobius pilicornis), nombre de chilopodes, notamment parmi les lithobiomorphes, sont enclins à pénétrer dans les milieux souterrains (entrées de grottes, zone intermédiaire ou zone profonde). Deux espèces de Lithobiomorpha s'étant même spécialisées dans le milieu cavernicole sont connues en Provence-Alpes-Côte d'Azur: les troglobiontes L. (L.) scotophilus Latzel, 1887 (Alpes-Maritimes) et L. (L.) fagniezi Ribaut, 1926 (Var). Ces derniers sont toutefois très éloignés morphologiquement de L. (L.) lemairei n. sp. (Latzel 1887; Manfredi 1948; Iorio 2008, 2010b). Une autre espèce cavernicole de Lithobius de la province de Cunéo, très probablement nouvelle, trouvée par Matic mais qui est restée inédite (A. Minelli comm. pers.), se rapproche de $L$. (L.) scotophilus tout en ayant aussi la particularité de posséder chez les deux sexes, sur la face latérale-postérieure des fémurs et surtout tibias et tarses des P15, un sillon longitudinal large, mais peu profond. Elle reste très différente de $L$. (L.) lemairei n. sp. sur plusieurs autres caractères importants, avec entre autres quatre à cinq petits ocelles pigmentés de chaque côté de la tête, des prolongements triangulaire très accentués aux tergites 9,11 et 13 , et une spinulation nettement plus fournie (A. Minelli comm. pers.).

Ainsi, seules quatre espèces du genre Lithobius se rapprochent de $L$. (L.) lemairei n. sp. sur le plan morphologique (Tableau 3) : en premier lieu, à cause de leurs structures très proches aux P15, L. (L.) guadarramus Matic, 1968 du Nord de l'Espagne et surtout L. (L.) longiscissus Serra, 1987 du Sud-Ouest de l'Espagne. Puis, en raison de leur plus grande proximité géographique et d'autres aspects morphologiques liés à leur étroite adaptation aux milieux souterrains profonds, les troglobiontes suivants: L. (L.) cherpinedensis Iorio, 2010 de Corse; L. (L.) electrinus Verhoeff, 1937 du Nord-Est de l'Italie, qui a été revu en détail pour cette description par l'un de nous (MZ) (Manfredi 1935, 1948; Verhoeff 1937; Matic 1957, 1967, 1968; Serra 1980, 1987; Salinas 1990; Zapparoli \& Minelli 2006; Iorio 2010b) ; ce dernier étant reconnu synonyme senior de L. binaghii Manfredi, 1955, L. italicus Matic, 1957, L. plumbeus Manfredi, 1948 et L. vignai Matic, 1967 (Minelli et al. 2013).

Bien que nous n'ayons pas encore pu examiner de mâle, la morphologie remarquable de $L$. (L.) lemairei $\mathrm{n}$. sp. autorise aisément des comparaisons fiables avec les taxons ci-dessus (cf. Tableau 3).

Lithobius (L.) lemairei n. sp. partage avec Lithobius (L.) longiscissus la particularité remarquable d'avoir un sillon longitudinal sur la face latérale-postérieure des fémurs, tibias, basitarses et tarses des P14 et des P15. Ce sillon est toutefois plus profond chez le second que chez le premier. Lithobius (L.) guadarramus possède également un tel sillon, mais uniquement sur les tibias, basitarses et tarses des P14 et des P15. Lithobius (L.) longiscissus et $L$. (L.) guadarramus, épigés, se différencient nettement de $L$. (L.) lemairei n. sp. par le fait de posséder au minimum quatre ocelles bien pigmentés. De plus, entre autres différences, L. longiscissus possède des prolongements denticulaires accentués au tergite 13 (T13), tandis que $L$. (L.) lemairei n. sp. en est dépourvu; $L$. (L.) guadarramus, quant à lui, possède un organe temporal nettement plus petit. Par la même structure remarquable décrite ci-dessus, soit celle du sillon longitudinal sur la face latérale-postérieure des fémurs, tibias, basitarses et tarses des P14 et des P15, il se distingue d'emblée des deux autres taxons, qui sont eux troglobies et complètement anophthalmes. En outre, le détail de la spinulation fournit également des éléments précieux pour séparer $L$. (L.) lemairei n. sp., L. (L.) cherpinedensis et $L$. (L.) electrinus (Tableau 3).

Enfin, il est intéressant de constater que dans les AlpesMaritimes, la seule autre espèce qui possède un sillon latéralpostérieur aux P15, mais uniquement aux basitarses et aux tarses, est L. (L.) pyrenaicus Meinert, 1872. Toutefois, cette 

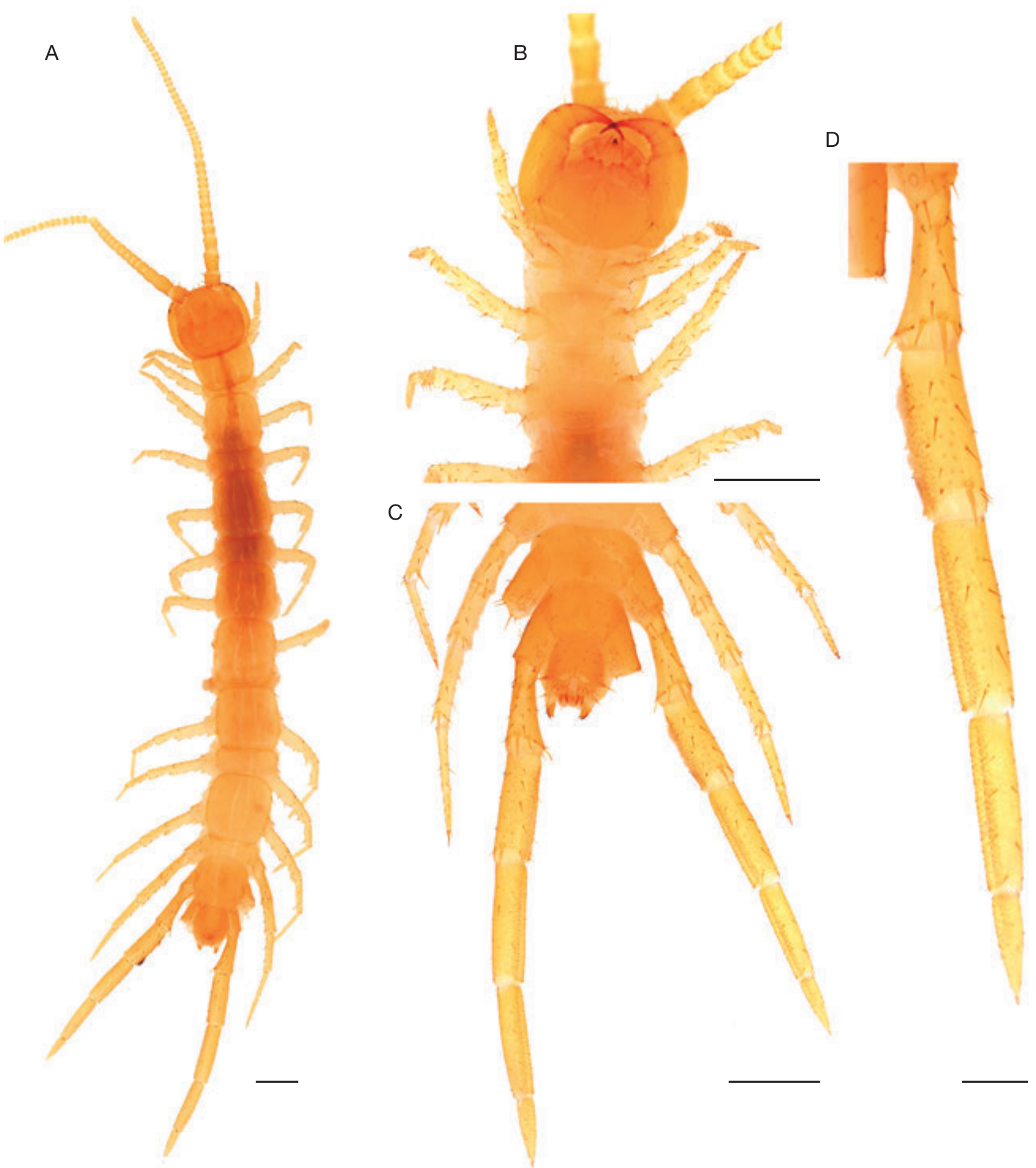

FIG. 1. - Lithobius (Lithobius) lemairei n. sp. (holotype femelle, $8 \mathrm{~mm}$ de long, d'Entrevaux): A, habitus, vue dorsale; $\mathbf{B}$, tête et premiers segments pédifères, vue ventrale; C, derniers segments pédifères et segments gonopodiaux, vue ventrale; D, patte 14 gauche, vue ventrale. Échelles : A-C, 0,5 mm; D, 0,2 mm.

grande espèce épigée (15 à $24 \mathrm{~mm}$ de long) diffère fortement de $L$. (L.) lemairei n. sp. par de nombreux autres caractères, notamment par la présence de 12 à 16 ocelles pigmentés et par la structure de la griffe gonopodiale de la femelle qui est constamment unidentée, complètement dépourvue de dentelures latérales (Iorio 2008, 2010b).
RÉPARTITION ET HABITAT. - Cette espèce remarquable était insoupçonnée. Nous l'avons récoltée dans un secteur rocheux et d'éboulis en pleine forêt, dans une partie un peu plus clairsemée de celle-ci. Ces enrochements et éboulis, en partie recouverts d'une couche de terre parfois épaisse et de feuilles mortes, voire de végétation, présentaient des fissures et micro-cavités cachées, de profondeurs difficilement estimables car certaines communiquaient avec un 
TABlEAU 3. - Principaux caractères de Lithobius (Lithobius) lemairei n. sp. comparés aux quatre espèces les plus proches d'après nos observations, Verhoeff (1937), Manfredi $(1935,1948)$, Matic $(1957,1967,1968)$, Serra $(1980,1987)$, Salinas (1990) et lorio (2010b). Abréviations : voir Matériel et méthodes.

\begin{tabular}{|c|c|c|c|c|c|}
\hline Caractères & L. Iemairei n. sp. & $\begin{array}{l}\text { L. cherpinedensis } \\
\text { lorio, } 2010\end{array}$ & $\begin{array}{l}\text { L. electrinus } \\
\text { Verhoeff, } 1937\end{array}$ & $\begin{array}{l}\text { L. guadarramus } \\
\text { Matic, } 1968\end{array}$ & $\begin{array}{l}\text { L. longiscissus } \\
\text { Serra, } 1987\end{array}$ \\
\hline Longueur du corps & $8 \mathrm{~mm}$ & $8,5 \mathrm{~mm}$ & $6-10 \mathrm{~mm}$ & $7-15 \mathrm{~mm}$ & $6,9-10,7 \mathrm{~mm}$ \\
\hline $\begin{array}{l}\text { Longueur moyenne } \\
\text { des antennes }\end{array}$ & $\begin{array}{l}\text { 2/5 de la longueur } \\
\text { du corps }\end{array}$ & $\begin{array}{l}\text { Entre } 2 / 5 \text { et } 1 / 2 \text { de } \\
\text { la longueur du corps }\end{array}$ & $\begin{array}{l}\text { 1/3 de la longueur } \\
\text { du corps }\end{array}$ & $\begin{array}{l}\text { Moins de } 1 / 2 \text { de } \\
\text { la longueur du corps }\end{array}$ & $\begin{array}{l}\text { Un peu plus de } 1 / 2 \text { de } \\
\text { la longueur du corps }\end{array}$ \\
\hline $\begin{array}{l}\text { Nombre d'articles } \\
\text { antennaires }\end{array}$ & $38-39$ & $38-41$ & $28-41$ & $29-57$ & $39-46$ \\
\hline Ocelles & $\begin{array}{l}\text { Microphthalme } \\
\text { «traces » de 1-2 } \\
\text { ocelles dépigmentés }\end{array}$ & Anophthalme & $\begin{array}{l}0 \\
\text { Anophthalme }\end{array}$ & 4-9 pigmentés & 5-6 petits et pigmentés \\
\hline $\begin{array}{l}\text { Organe de } \\
\text { Tömösváry }\end{array}$ & $\begin{array}{l}\text { De grande taille, } \\
\text { relativement } \\
\text { sclérifié, beaucoup } \\
\text { plus grand et visible } \\
\text { que les ocelles }\end{array}$ & $\begin{array}{l}\text { De très grande taille } \\
\text { mais peu sclérifié, } \\
\text { peu visible }\end{array}$ & $\begin{array}{l}\text { De très grande taille } \\
\text { et bien sclérifié, } \\
\text { très net, atteignant } \\
\text { environ } 1 / 3 \text { de la } \\
\text { largeur de l'article } \\
\text { basal des antennes }\end{array}$ & $\begin{array}{l}\text { De petite taille, } \\
\text { égale à celle } \\
\text { d'un des plus petits } \\
\text { ocelles }\end{array}$ & $\begin{array}{l}\text { D'assez grande } \\
\text { taille, plus grand } \\
\text { que le plus grand } \\
\text { des ocelles }\end{array}$ \\
\hline $\begin{array}{l}\text { Nombre de dents } \\
\text { forcipulaires }\end{array}$ & $2+2$ & $2+2$ & $2+2$ & $2+2$ & $2+2$ \\
\hline $\begin{array}{l}\text { Prolongements } \\
\text { triangulaires } \\
\text { tergaux }\end{array}$ & Aucun & Aucun & Aucun & Aucun & $\begin{array}{l}\text { Absents au T9; infîmes } \\
\text { au T11; prononcés } \\
\text { au T13 }\end{array}$ \\
\hline Pores coxaux & 2 & $2-3$ & $2-3$ & 2 & $2-4$ \\
\hline $\begin{array}{l}\text { Épine coxolatérale } \\
\quad(\mathrm{VaH})\end{array}$ & Absente & Absente & Absente & Absente & Présente \\
\hline $\begin{array}{l}\text { Structures spéciales } \\
\text { aux P14 et/ou aux } \\
\text { P15 }\end{array}$ & $\begin{array}{l}\text { Fémurs, tibias, } \\
\text { basitarses et } \\
\text { tarses des P14 et } \\
\text { des P15 dotés, sur } \\
\text { leur face latérale- } \\
\text { postérieure, d'un } \\
\text { sillon longitudinal } \\
\text { modérément } \\
\text { prononcé }\end{array}$ & $\begin{array}{l}\text { Aucune } \\
\text { chez la femelle } \\
\text { (mâle inconnu) }\end{array}$ & Aucune & $\begin{array}{l}\text { Tibias, basitarses } \\
\text { et tarses des P14 } \\
\text { et des P15 dotés, } \\
\text { sur leur face } \\
\text { latérale-postérieure, } \\
\text { d'un sillon } \\
\text { longitudinal très } \\
\text { prononcé }\end{array}$ & $\begin{array}{l}\text { Fémurs, tibias, } \\
\text { basitarses et tarses } \\
\text { des P14 et des P15 } \\
\text { dotés, sur leur } \\
\text { face latérale- } \\
\text { postérieure, d'un } \\
\text { sillon longitudinal } \\
\text { prononcé }\end{array}$ \\
\hline Plectrotaxie & $\begin{array}{l}\text { VaF débute P4 } \\
\text { et manque aux P14; } \\
\text { VpF sur P12 et P13; } \\
\text { VaT existe de P8/ } \\
\text { P9 jusqu'aux P13; } \\
\text { DaH aux P15; DpF } \\
\text { jusqu'aux P13 }\end{array}$ & $\begin{array}{l}\text { VaF débute dès P2 et } \\
\text { manque aux P14; } \\
\text { aucune VpF; aucune } \\
\text { VaT; DaH sur P14 et } \\
\text { P15; DpF jusqu'aux } \\
\text { P13 }\end{array}$ & $\begin{array}{l}\text { VaF débute } \\
\text { généralement entre } \\
\text { P6 et P10, et existe } \\
\text { toujours aux P14; } \\
\text { VpF au moins sur } \\
\text { P13, voire sur P12 } \\
\text { et P13; aucune VaT; } \\
\text { aucune DaH ou DaH } \\
\text { juste aux P15; DpF } \\
\text { jusqu'aux P14 }\end{array}$ & $\begin{array}{l}\text { VaF débute P1 et } \\
\text { existe jusqu'aux } \\
\text { P15; VpF existe } \\
\text { généralement } \\
\text { de P1 à P14; VaT } \\
\text { existe de P1 à P14 } \\
\text { (voire P15); DaH } \\
\text { sur P13 à P15; DpF } \\
\text { jusqu'aux P15 }\end{array}$ & $\begin{array}{l}\text { VaF débute P1 et } \\
\text { existe jusqu'aux } \\
\text { P15; VpF existe de } \\
\text { P1 à P13; VaT existe } \\
\text { de P1 à P15; DaH } \\
\text { sur P12 à P15; DpF } \\
\text { jusqu'aux P15 }\end{array}$ \\
\hline $\begin{array}{l}\text { Griffe apicale aux } \\
\quad \text { P15 }\end{array}$ & Simple & Simple & $\begin{array}{l}\text { Simple ou double } \\
\text { (griffe annexe } \\
\text { très petite) }\end{array}$ & Simple & Simple \\
\hline Gonopodes femelles & $\begin{array}{l}2+2 \text { éperons, } \\
\text { griffe tridentée }\end{array}$ & $\begin{array}{l}2+2 \text { éperons, } \\
\text { griffe tridentée }\end{array}$ & $\begin{array}{l}2+2 \text { éperons, } \\
\text { griffe tridentée }\end{array}$ & $\begin{array}{l}2+2 \text { éperons } \\
\text { (rarement } \\
2+3 \text { ou } 3+3), \\
\text { griffe bidentée }\end{array}$ & Femelle inconnue \\
\hline
\end{tabular}

réseau plus profond. En soulevant terre et grosses pierres qui masquaient une de ces fissures, nous y avons récolté l'unique spécimen qui devait initialement se trouver à quelques dizaines de centimètres sous le sol. D'autres enrochements potentiellement fracturés et/ou éboulis existent à proximité sur les mêmes reliefs, vallons et pentes calcaires. Ces habitats assimilables à un milieu souterrain superficiel (Juberthie et al. 1981), présentent un réseau de diaclases et de micro-cavités étendu sur plusieurs mètres de profondeur et inaccessible à l'homme. Ils sont bien isolés de la lumière et conservent probablement une humidité supérieure et une température moins variable qu'à l'extérieur. Le peuplement d'arthropodes de cet habitat original a d'ailleurs fait récemment l'objet de nombreuses recherches en Europe centrale, avec des résultats tout à fait remarquables (Růžička et al. 2012).
Cette espèce se trouvait à proximité de la surface probablement en raison de conditions particulières (bon isolement des micro-cavités). Il s'agit sans doute d'un cas d'endémisme national et local en raison de son mode de vie, peu favorable à une large dispersion. De plus, le bon niveau de connaissance et les nombreuses cavités explorées dans les grands secteurs géographiques plus au sud et surtout à l'est permettent de penser qu'elle n'y est pas présente (Caziot 1925; Jeannel 1926; Manfredi 1932; Zapparoli 1980, 1993; Minelli \& Zapparoli 1985, 1992; Iorio 2008, 2010a, 2010b).

Lithobius (Lithobius) lucifugus L. Koch, 1862

Lithobius lucifugus L. Koch, 1862: 82. 


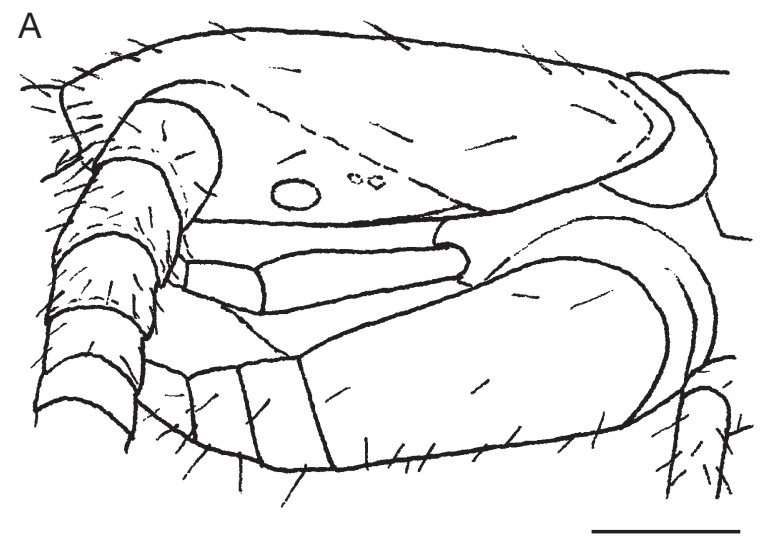

B
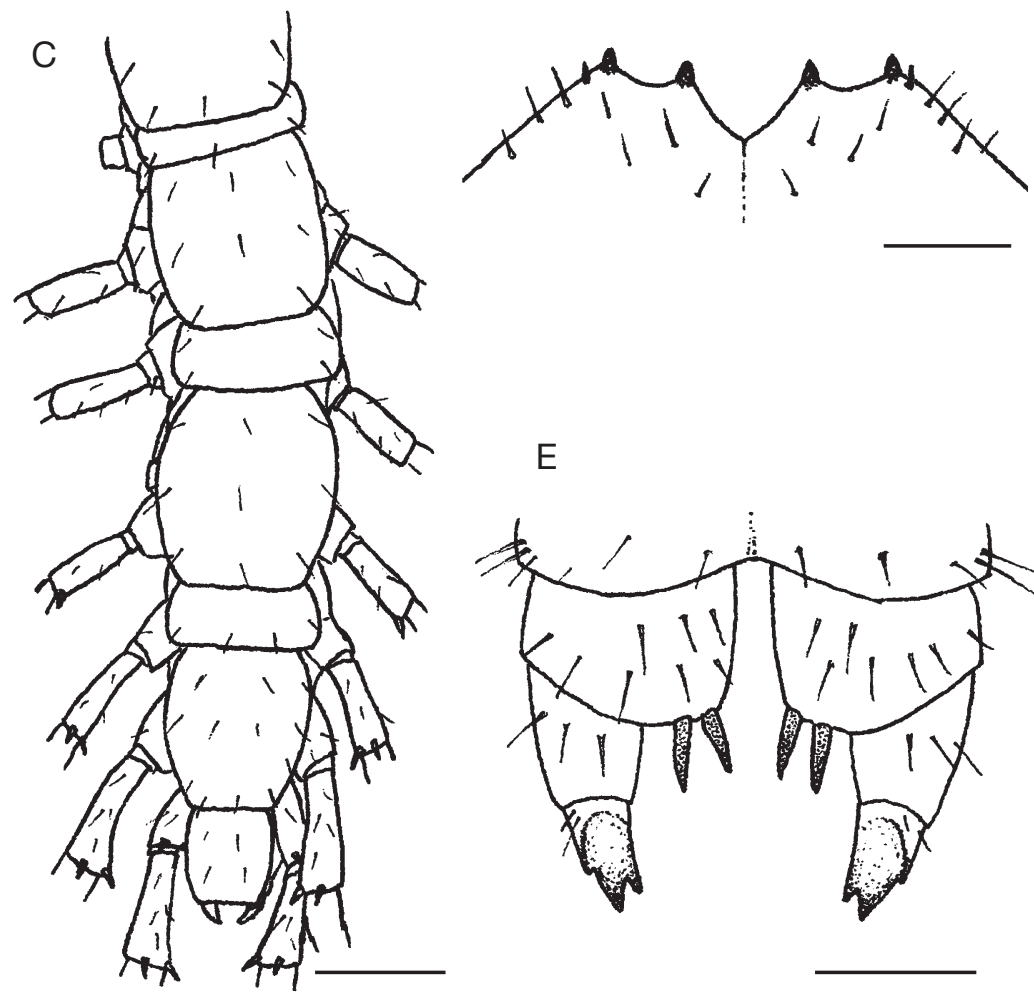

D
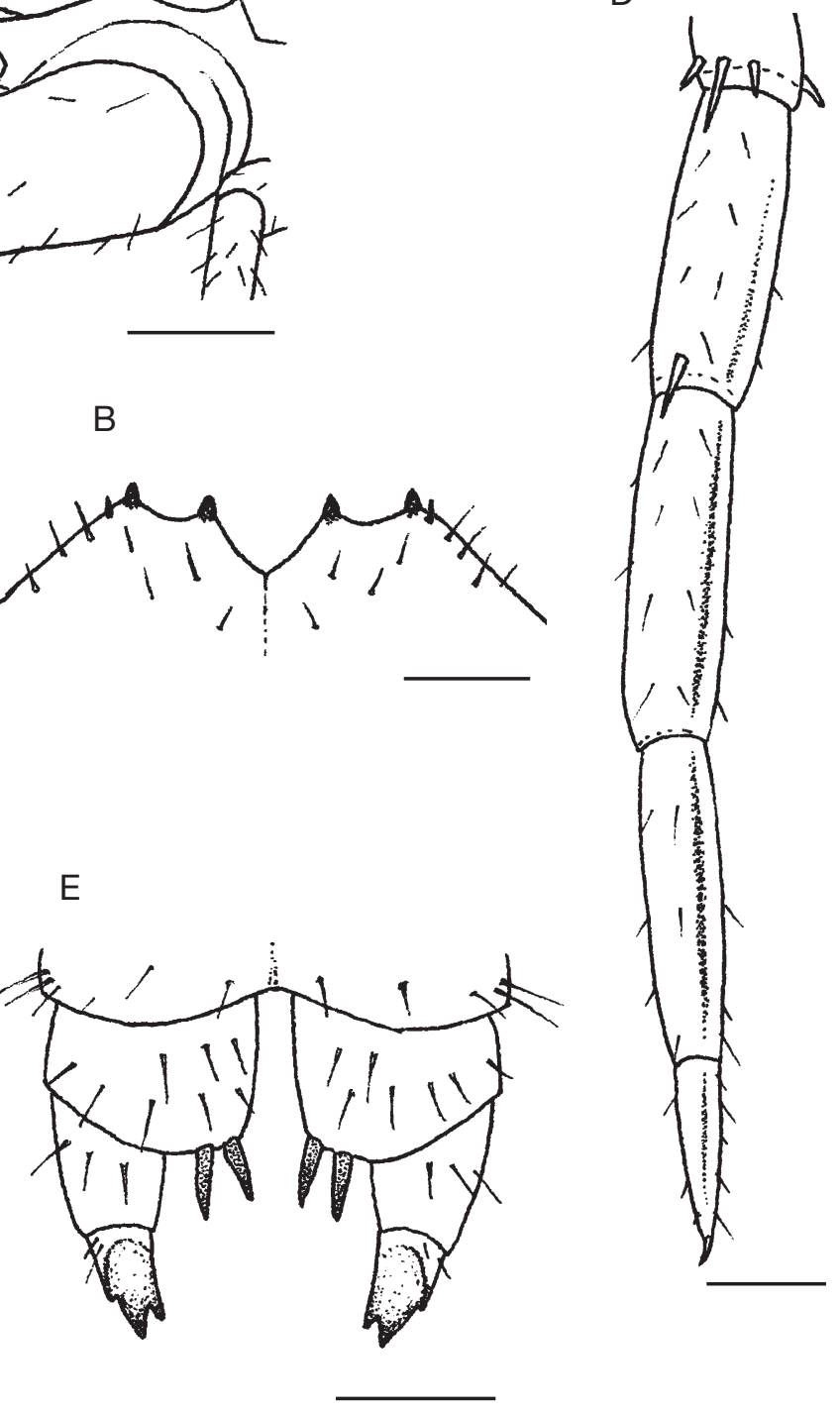

FIG. 2. - Lithobius (Lithobius) lemairei n. sp. (holotype femelle, $8 \mathrm{~mm}$ de long, d'Entrevaux). A, tête, vue latérale; B, bord rostral du coxosternum forcipulaire, vue ventrale; $\mathbf{C}$, moitié postérieure du corps, vue dorsale; $\mathbf{D}$, patte 15 droite, vue ventrale (pores glandulaires non représentés); $\mathbf{E}$, gonopodes, vue ventrale. Échelles : A, D : $0,2 \mathrm{~mm}$; B, E : $0,1 \mathrm{~mm}$; C : $0,5 \mathrm{~mm}$.

\section{LOCALITÉ TYPE. — Italie, Bolzano.}

Matériel eXAminé. - Lucéram (06), Peira-Cava, La Cabanette, hêtres-conifères, 1350-1400 m, 1.X.2008, réc./dét. EI : 3 \% ; SaintDalmas-le-Selvage (06), PNM, La Buisse, lisière d'un bois, en surface, $1437 \mathrm{~m}, 44.2848357 \mathrm{~N}, 6.8875257 \mathrm{E}, 17 . V I I I .2009$, réc./ dét. JJG : 1 9 ; Saint-Martin-Vésubie (06), PNM, vallon Madone de Fenestre, ubac, sapinière-pessière, près d'une petite rivière, $1700 \mathrm{~m}$, $44^{\circ} 05^{\prime} 26^{\prime \prime} \mathrm{N}-7^{\circ} 20^{\prime} 48^{\prime \prime} \mathrm{E}, 22 . \mathrm{V} .2010$, réc./dét. MZ: $30^{\prime \prime}, 2$ \% ; SaintMartin-Vésubie (06), PNM, vallon Madone de Fenestre, lariçaie, $1830 \mathrm{~m}, 4^{\circ} 05^{\prime} 36^{\prime \prime N}-7^{\circ} 21^{\prime 2} 24^{\prime \prime E}, 22 . V .2010$, réc./dét. MZ: $10^{\prime \prime}$, 49 ; Saint-Martin-Vésubie (06), PNM, vallon Madone de Fenestre, adret, $1170 \mathrm{~m}, 44^{\circ} 04^{\prime} 51^{\prime \prime N}-7^{\circ} 16^{\prime} 09^{\prime} \mathrm{E}, 22 . V .2010$, réc./dét. MZ: $20^{\circ}, 1$. Spécimens conservés en éthanol à $70^{\circ}$ dans la collection personnelle des auteurs (EI, MZ) ou dans celle du MNHN (Myriapoda, Lithobiomorpha) (JJG).

RÉPARTITION ET HABITAT. — Cette espèce est essentiellement présente en Russie et en Europe centrale, jusqu'en France où elle n'est formellement connue que dans le Sud-Est (Geoffroy \& Iorio 2009), sa présence dans le Nord-Est restant possible mais incertaine (Iorio 2007). Six des stations du département des Alpes-Maritimes où cette espèce a été identifiée fournissent des informations sur les habitats: cinq forêts dont une châtaigneraie, deux mixtes (pins-sapins-hêtres) et une lariçaie; une pelouse subalpine. Elle apparaît comme une espèce monticole, presque toujours trouvée au minimum à 1000 mètres d'altitude (Iorio 2008, 2010b), y compris dans le Nord de l'Italie (Minelli \& Zapparoli 1985, 1992; Minelli \& Iovane 1987; Minelli 1992; Zapparoli 1993, 2009a). 
Lithobius (Lithobius) mutabilis L. Koch, 1862

Lithobius mutabilis L. Koch, 1862: 21.

LOCALITÉ TYPE. - Allemagne, Jura franconien.

Matériel eXaminé. - Saint-Martin-Vésubie (06), PNM, Forêt de Boréon, parking 5, forêt mixte de conifères, $1500 \mathrm{~m}$, $44^{\circ} 06^{\prime} 59^{\prime \prime} \mathrm{N}-7^{\circ} 16^{\prime} 44^{\prime \prime E}, 21 . V .2010$, réc. AVT/dét. MZ: $20^{\circ}$. Spécimens conservés en éthanol à $70^{\circ}$ dans la collection personnelle de l'auteur (MZ).

RÉPARTITION ET HABITAT. - Espèce surtout centre-européenne, qui atteint l'Est de la France et même, d'après des données historiques, la Haute-Normandie (Geoffroy \& Iorio 2009). Elle apparaît toutefois comme très rare dans le Sud-Est, notamment en Provence-AlpesCôte d'Azur, ainsi que dans le Nord-Ouest de l'Italie (Zapparoli \& Minelli 2006). La citation de L. (L.) mutabilis à Cannes par Caziot (1925) paraissait douteuse (Iorio 2008), mais la présente observation à Saint-Martin-Vésubie confirme sa présence dans le département des Alpes-Maritimes. À l'image de l'unique habitat d'où elle est attestée, cette espèce y est très probablement sylvicole à l'instar de toutes les autres contrées où elle est connue (Iorio 2014).

\section{Lithobius (Lithobius) nicoeensis Brölemann, 1904}

Lithobius nodulipes nicoeensis Brölemann, 1904: 12.

LOCALITÉ TYPE. - Monaco ; vallée de la Siagne jusqu’à la frontière italienne.

Matériel EXAMINÉ. - Valbonne (06), Sophia-Antipolis, lisières de pinèdes, $150 \mathrm{~m}, 43,638794 \mathrm{~N}, 7,044013 \mathrm{E}, 10 . I V .2012$, réc./ dét. EI : 1 o ; Roquebrune-Cap-Martin (06), Mont Gros, flanc d'exposition nord-est, chênaie, 500-550 m, 30.X.2012, 43.7675N, 7.44138E, réc./dét. EI : 1 o ; idem, 43.7681N, 7.44454E, réc./dét. EI : $10^{\pi}$; Monaco, aven, XI-XII.2012, réc. JML/dét. EI : 1 \% ; idem, I-III.2013: $20^{\circ}, 2$. . Spécimens conservés en éthanol à $70^{\circ}$ dans la collection personnelle de l'auteur (EI).

RÉPARTITION ET HABITAT. - Cette espèce vit en Italie et dans l'extrême Sud-Est de la France (Iorio 2008; Geoffroy \& Iorio 2009). Nous avons récemment trouvé $L$. (L.) nicoeensis dans l'Est du Var, à Fréjus (talus herbeux mésophile en lisière d'une route et de bosquets arborés, $43,4814^{\circ} \mathrm{N}, 6,74595^{\circ} \mathrm{E}$, IV.2010, réc./dét. EI : 1 ㅇ) , ce qui constitue une première observation dans ce département. Cette espèce est connue dans d'assez nombreuses stations du littoral des Alpes-Maritimes, mais seules huit stations récentes renseignent sur les habitats: une près d'une plage, une dans une forêt de feuillus divers, une dans une chênaie, une dans une garrigue à chênes verts, une dans une lisière de pinède, trois dans ou à proximité immédiate d'une grotte ou aven. Par contre, il semble que cette espèce ne vive qu'à basse altitude ( 0 à 800 mètres maximum dans les Alpes-Maritimes).

\section{REMARQUES}

La synonymie de $L$. (L.) nicoeensis avec $L$. (L.) tylopus Latzel, 1882 suggérée par Minelli et al. (2013) reste incertaine. Nous conservons donc ici $L$. $(L$.) nicoeensis en tant que taxon valide, d'autant qu'il a déjà été montré que deux taxons du genre Lithobius morphologiquement très proches pouvaient se distinguer par le seul caractère de la griffe apicale des P15 (cf. les cas de $L$. (L.) tricuspis Meinert, 1872 et $L$. (L.) mononyx Latzel, 1888) (Eason 1974; Iorio 2010b, 2014).

\section{Lithobius (Lithobius) piceus verhoeffi \\ Demange, 1958}

Lithobius piceus verhoeff Demange, 1958: 853.

LoCAlité TYPE. - Suisse, Gitziloch (BE 13), commune de Court, canton de Berne (Jura)

MATÉRIel eXAminé. - Saint-Dalmas-le-Selvage (06), PNM, La Buisse, lisière d'un bois, en surface, $1437 \mathrm{~m}, 44.2848357^{\circ} \mathrm{N}$, 6.8875257 $\mathrm{E}, 17$. VIII.2009, réc./dét. JJG : 1 \% ; Mont Gros, flanc d'exposition nord-est, chênaie, $500-550 \mathrm{~m}, 30 . X .2012,43.7681^{\circ} \mathrm{N}$, $7.44454^{\circ} \mathrm{E}$, réc./dét. EI : 1 \% ; Menton (06), à proximité du Chemin des Granges de Saint-Paul, dans une pinède, 400-450 m, 24.X.2013, réc./dét. EI: $10^{\circ}$. Spécimens conservés en éthanol à $70^{\circ}$ dans la collection personnelle d'un des auteurs (EI) ou dans celle du MNHN (Myriapoda, Lithobiomorpha) (JJG).

RÉPARTITION ET HABITAT. - Cette sous-espèce remplace $L$. (L.) piceus piceus L. Koch, 1862 dans le Sud-Est de la France, en Suisse et dans le Nord de l'Italie (Zapparoli \& Minelli 2006; Geoffroy \& Iorio 2009; Stöckli 2009); une aire de chevauchement entre les deux sous-espèces existe dans le nord des Alpes (Iorio 2010b). À l'instar de la forme typique, $L$. (L.) piceus verhoeffi semble privilégier les forêts, car les stations des Alpes-Maritimes pour lesquelles des données écologiques existent sont toutes des milieux boisés (Iorio 2008, $2010 \mathrm{~b}$; présent travail) : une châtaigneraie, une forêt de feuillus divers dominée par le châtaignier, une lariçaie, une chênaie, une pinède, une lisière d'un bois. Elle est présente de 400 à 1437 mètres d'altitude dans les Alpes-Maritimes.

\section{Lithobius (Lithobius) pilicornis pilicornis Newport, 1844}

Lithobius pilicornis Newport, 1844: 96.

LOCALITÉ TYPE. - Angleterre.

Matériel EXAMinÉ. - Beuil (06), PNM, Les Launes, conifères et lisière pelouse, $1600 \mathrm{~m}, 7 . V I .2008$, réc./dét. EI : $10^{7}, 2$; ; Lucéram (06), Peira-Cava, La Cabanette, hêtres-conifères, 1350-1400 m, 1.X.2008, réc./dét. EI : 1 ơ⿱ $^{\text {, }}$ \% ; Saint-Martin-Vésubie (06), PNM, Le Boréon, 1520 m, réc. SS/dét. JJG, 44.1170379N, 7.2787055E, 11.VI.2009: $10^{7}$ immature; Saint-Martin-Vésubie (06), PNM, vallon de Champouns, rivière à sec, pierres, $1047 \mathrm{~m}, 44.060011 \mathrm{~N}$, 7.2475272E, 11.VI.2009, réc. SS/dét. JJG: 10, 19; Lantosque (06), Loda, bord de rivière, pierres, 12.VI.2009, réc. SS/dét. JJG: 19; Entrevaux (04), à proximité de Combe Guénier, 900-1000 m d'altitude, boisement de conifères et de hêtres, VII.2009, réc./ dét. EI : $20^{7}, 1$ 우 ; Saint-Dalmas-le-Selvage (06), PNM, forêt de Sestrière, mélèzaie, au sol, 1995 m, 44.2926706N, 6.8235944E, 17.VIII.2009, réc./dét. JJG: $10^{7}$; Péone (06), PNM, Gîte d'Aliège, prairies et garrigues boisées, friches, $1400 \mathrm{~m}, 18$.VIII.2009, réc./ dét. JJG: $10^{\prime \prime}, 2$ \% ; Colmars-les-Alpes (04), PNM, Ratery, forêt domaniale de la Haute-Vallée du Verdon, mélèzaie, sol et bois mort, 1700 m, 22.VIII.2009, réc./dét. JJG : 10 $0^{7}$, 1\%; Allos (04), PNM, Lac d'Allos, landes et pelouses alpines, mélèzes, $2230 \mathrm{~m}$, $44^{\circ} 14^{\prime} 0^{\prime} \mathrm{N}, 6^{\circ} 42^{\prime} 30^{\prime} \mathrm{E}, 23$.VIII.2009, réc./dét. JJG: $10^{*}, 1$ \% ; Mandelieu (06), garrigue, en lisière arborée (chênes), $16 \mathrm{~m}, 43,528558 \mathrm{~N}$, 6,914716E, 7.V.2010, réc./dét. EI : 19 ; Saint-Martin-Vésubie (06), PNM, Forêt de Boréon, parking 5, forêt mixte de conifères, $1500 \mathrm{~m}, 44^{\circ} 06^{\prime} 59^{\prime \prime N}-7^{\circ} 16^{\prime} 44^{\prime \prime E}, 21 . V .2010$, réc./dét. MZ: $10^{\prime \prime}, 10^{\prime \prime}$ immature; Saint-Martin-Vésubie (06), PNM, vallon Madone de Fenestre, ubac, $1600 \mathrm{~m}, 44^{\circ} 05^{\prime} 25^{\prime \prime} \mathrm{N}-7^{\circ} 19^{\prime} 53^{\prime \prime} \mathrm{E}, 22 . \mathrm{V} .2010$, réc./ dét. MZ: $20^{7}$; Saint-Martin-Vésubie (06), PNM, vallon Madone de Fenestre, ubac, sapinière-pessière, près d'une petite rivière, $1700 \mathrm{~m}$, $44^{\circ} 05^{\prime} 26^{\prime \prime} \mathrm{N}-7^{\circ} 20^{\prime} 48^{\prime \prime} \mathrm{E}, 22 . \mathrm{V} .2010$, réc./dét. MZ: $30^{\prime \prime}, 1$ 우 ; Saint- 
Martin-Vésubie (06), PNM, vallon Madone de Fenestre, lariçaie, $1830 \mathrm{~m}, 4^{\circ} 05^{\prime} 36^{\prime \prime} \mathrm{N}-7^{\circ} 21^{\prime} 24$ ”E, 22.V.2010, réc./dét. MZ: $10^{\prime \prime}, 1$ \% 1 immature; Saint-Martin-Vésubie (06), PNM, vallon Madone de Fenestre, milieux ouverts à proximité d'un boisement de genévriers,

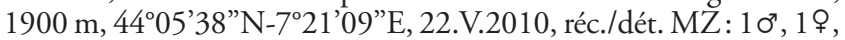
$10^{7}$ immature; Lucéram (06), grotte de Peira-Cava, 30.VII.2010, réc. JML \& JR/dét. EI : 1 ; ; Larche (04), PNM, Tête de Viraysse, sommet rocheux, $2750 \mathrm{~m}, 14$.VI.2010, réc./dét. FB : 1 \% ; Tende (06), PNM, Granile, grotte de Fouige, 2010-2011, réc. JML/dét. EI : $10^{7}, 3$; Peille (06), Roc Agel, source aux Niphargus, 2011, réc. JML/dét. EI: 10, 19; Calern (06), aven du Cerisier, 2011, réc. JML/dét. EI : $10^{7}, 1$ \% ; Larche (04), PNM, Cime de la Coste du Col, 44,47536N, 6,88587E, $2842 \mathrm{~m}, 02$.VIII.2011, réc./dét. FB: 1 ex. ; Valbonne (06), Sophia-Antipolis, pins, chênes verts et chênes pubescents, $236 \mathrm{~m}, 43,626743 \mathrm{~N}, 7,036508 \mathrm{E}, 11 . I V .2012$, réc./dét. EI : $10^{7}$; Digne-les-Bains (04), Le Serre, $1100 \mathrm{~m}$, prairie humide, 27.V.2012, réc. PF/dét. EI: 2 q dont une immature; Guillaumes (06), PNM, grotte de Tremens, 2012, réc. JML/dét. EI : $10^{7}, 2$; Castellet-les-Sausses (04), col du Fâ, grotte de la Kalams, 2012-2013, réc. JML/dét. EI : $10^{7}, 7$; ; Venanson (06), aven du Saint-Esprit (n¹56B), 2012-2013, réc. JR/dét. EI : $30^{*}, 3$ \% ; Roquefort-lesPins (06), Baume Granet, dans la grotte à 20 mètres de l'entrée, 22.V.2013, réc./dét. EI : $20^{7}, 2$ \% ; Belvédère (06), PNM, vallée de Gordolasque, $2200 \mathrm{~m}, 29$.VII.2013, réc. MT/dét. EI : $10^{7}$; Peille (06), aven de Faille, 816 m, 5.IX.2013, réc. JML/dét. EI : 1 \% ; Peille (06), grotte de Morgelle (dite "Saint-Jean »), 2013, réc. JML/dét. EI : 19 . Spécimens conservés en éthanol à $70^{\circ}$ dans la collection personnelle des auteurs (EI, MZ) ou dans celle du MNHN (Myriapoda, Lithobiomorpha) (JJG).

RÉPARTITION ET HABITAT. - Lithobius (L.) pilicornis pilicornis, ouest-européen, assez largement réparti en France où il manque toutefois dans le quart Nord-Est et plus à l'est en Allemagne (Geoffroy \& Iorio 2009), est le Lithobiidae le plus commun des AlpesMaritimes, où on le trouve quasiment partout (Iorio 2008, 2010b; présent travail). Parmi les stations énumérées dans ce département, l'habitat est connu pour 86 d'entre elles (Iorio 2008, 2010b; présent travail) : neuf châtaigneraies, neuf pinèdes, cinq lariçaies, cinq forêts indéfinies, quatre sapinières-pessières, trois pessières, trois forêts de feuillus variés dont deux dominées par le châtaignier, deux hêtraies-conifères divers, une châtaigneraie-corylaie, une aulnaie, une ripisylve indéfinie, une sapinière, un boisement de conifères divers, un boisement de genévriers, une pinède avec chênes verts et pubescents, une lisière arborée (chênes) de garrigue, une lisière de coniferes/pelouse, 15 prairies/pelouses dont six alpines, quatre subalpines, une à rhodoraie, trois montagnardes, une alternée avec garrigue, deux landes thermophiles, deux au bord d'un lac ou d'une rivière, une dans une rivière asséchée, une sommet rocheux (nival), deux vallons obscurs et enfin 14 grottes ou avens.

\section{REMARQUES}

Trois des quatre spécimens de la grotte de Fouige, notamment une femelle, se rapprochent plus ou moins de $L$. (L.) pilicornis luridus Serra, 1981, sous-espèce troglobie décrite de cavités du Nord de l'Espagne (provinces de Guipuscoa et de Navarre) (Serra 1980, 1981). En effet, pour la femelle chez qui les affinités sont les plus grandes, les antennes ont leurs articles nettement plus allongés que chez les spécimens épigés (une des antennes de cette femelle atteint $18 \mathrm{~mm}$ pour une longueur corporelle totale de $30,5 \mathrm{~mm}$ ), une douzaine d'ocelles (très) réduits et dépigmentés, et un organe de Tömösváry bien plus grand que le plus grand des ocelles. Les P15 atteignent $15,3 \mathrm{~mm}$, et la griffe des $\mathrm{P} 15$ est plus longue qu'à l'accoutumée, de même que les épines ventro-médianes de la plupart des pattes. Un des spécimens (un mâle) correspond davantage à la sous-espèce typique, même si les ocelles sont plus désorganisés et certains articles des antennes plus longs chez celui-ci. Les deux autres constituent des intermédiaires entre la femelle brièvement décrite et le mâle. Contrairement aux populations de la sous-espèce luridus, les individus de la grotte de Fouige ne sont pas aussi évolués vers une adaptation cavernicole, puisque même au sein de la population de cette grotte, les individus présentent des évolutions morphologiques variées, soit quasi-identiques à la sous-espèce typique, soit se rapprochant plus ou moins de la sous-espèce de Serra sans qu'il s'agisse d'aspects et proportions aussi marqués (Serra 1980, 1981). Il serait toutefois intéressant d'effectuer de futurs prélèvements à divers niveaux de profondeur de la grotte de Fouige, afin de déterminer si les individus les plus en profondeur présentent systématiquement des particularités morphologiques comme celles de la femelle la plus éloignée du type. En attendant, il est vraisemblable que la population de Fouige puisse à terme évoluer vers une vie exclusivement cavernicole en profondeur, et être isolée de l'espèce typique; cette dernière étant épigée mais présentant une certaine propension à entrer dans les cavités tout en restant généralement près de l'entrée comme nous avons pu le constater à plusieurs reprises. À noter que les mêmes particularités, mais de façon tout de même moins marquées que chez la femelle la plus éloignée de la forme typique de Fouige, s’observent également chez les huit spécimens de la grotte de la Kalams, ainsi que chez les six exemplaires de l'aven du Saint-Esprit.

Une situation semblable s'observe dans la grotte de Nabrigas (Lozère, Causse Méjean), au sein de spécimens appartenant à une population de $L$. (L.) pilicornis présentant les mêmes caractéristiques troglomorphes que celles de L. (L.) pilicornis luridus et témoignant d'une forme de spéciation en cours au sein des peuplements cavernicoles des chilopodes des Grands Causses (Geoffroy 1981a, 1982). De même que pour ceux de la grotte de Fouige, les spécimens de la grotte de Nabrigas mériteraient de faire l'objet d'une étude morphologique approfondie, associée à une étude génétique.

\section{Lithobius (Lithobius) pyrenaicus Meinert, 1872}

Lithobius pyrenaicus Meinert, 1872: 337.

LOCALITÉ TYPE. — Espagne, Gérone.

MATÉRIEL EXAMINÉ. - Mandelieu (06), garrigue, en lisière arborée (chênes), 16 m, 43,528558N, 6,914716E, 7.V.2010, réc./dét. EI: $10^{7}$; Valbonne (06), Sophia-Antipolis, friches/lisières de pinèdes, $150 \mathrm{~m}, 43,638794 \mathrm{~N}, 7,044013 \mathrm{E}, 10 . I V .2012$, réc./dét. EI : 1 \% ; Valbonne (06), Sophia-Antipolis, pins, chênes verts et chênes pubescents, $236 \mathrm{~m}, 43,625116 \mathrm{~N}, 7,031673 \mathrm{E}, 11 . \mathrm{IV} .2012$, réc./ dét. EI : $10^{\prime \prime}$. Spécimens conservés en éthanol à $70^{\circ}$ dans la collection personnelle de l'auteur (EI).

RÉPARTITION ET HABITAT. —Ce lithobiomorphe méditerranéen francoibérique est en limite orientale d'aire de répartition dans le département des Alpes-Maritimes (Iorio 2008, 2010b; Geoffroy \& Iorio 2009). Malgré les nombreuses études menées sur les chilopodes dans le Nord de l'Italie, il n’y a jamais été trouvé (cf. Minelli \& Zapparoli 1985, 1992; Minelli 1992; Zapparoli 1993, 2009a; Zapparoli \& Minelli 2006). Nous avons identifié cette espèce dans le sud des Hautes-Alpes, 
à Ribiers (pelouse sèche, 8.IV.2011, réc. SB/dét. EI : $10^{7}$ ), ce qui constitue une première citation pour ce département, et la nouvelle limite septentrionale connue de son aire. Dans les Alpes-Maritimes proprement dites, peu d'informations écologiques sont disponibles. Toutefois, sur l'ensemble du littoral méditerranéen, $L$. (L.) pyrenaicus apparaît comme une espèce xéro-thermophile (Iorio 2014).

\section{Lithobius (Lithobius) tricuspis Meinert, 1872}

\section{Lithobius tricuspis Meinert, 1872: 298.}

LOCALITÉ TYPE. - Allemagne, Sydtydskland, Tubingen; Italie, Razzes, Italie centrale, Villa Chigi i Ariccia, Gennazano, Campagne romaine.

MatéRIEL EXAMINÉ. — Guillaumes (06), PNM, Le Coulet, conifères divers, 1470-1500 m, 8.VI.2008, réc./dét. EI : 1 \% ; Le Fugeret (04), Trou de Baude, XII.2007-VIII.2008, réc. JML \& JR/dét. EI : 2 ㅇ ; Lucéram (06), Peira-Cava, La Cabanette, hêtres-conifères, 13501400 m, 1.X.2008, réc./dét. EI: 1 우 ; Saint-Auban (06), faille du Pensier, 2.V.2009, réc. JML \& JR/dét. EI : 4 \% ; Entrevaux (04), à proximité de Combe Guénier, 900-1000 m d'altitude, boisement de conifères et de hêtres, VII.2009, réc./dét. EI : 1 \% ; Colmars-lesAlpes (04), PNM, Ratery, forêt domaniale de la Haute-Vallée du Verdon, mélèzaie, sol et bois mort, $1700 \mathrm{~m}, 22$.VIII.2009, réc./ dét. JJG : 1 \% ; Saint-Martin-Vésubie (06), PNM, vallon Madone de Fenestre, ubac, sapinière-pessière, près d'une petite rivière, $1700 \mathrm{~m}$, $44^{\circ} 05^{\prime} 26^{\prime \prime} \mathrm{N}-7^{\circ} 20^{\prime} 48^{\prime \prime} \mathrm{E}, 22 . V .2010$, réc./dét. MZ: 1 ㅇ ; Saint-Martin-Vésubie (06), PNM, Forêt de Boréon, parking 5, forêt mixte de conifères, $1500 \mathrm{~m}, 4^{\circ} 06^{\prime} 59^{\prime \prime} \mathrm{N}-7^{\circ} 16^{\prime} 44^{\prime \prime E}, 21 . V .2010$, réc./dét. MZ: 60", 69 ; Prads-Haute-Bléone (04), Pic des Têtes, $2661 \mathrm{~m}$, 44,30084N, 6,46757E, 10.IX.2010, réc./dét. FB : 1 우 ; Valdeblore (06), PNM, Cluchelier inférieur (ancienne mine), 1400-1450 m, 2010, réc. JML/dét. EI : 2 \% Calern (06), Cipières, 25.V.2011, tamisage, réc. JML/dét. EI : 1 i immature. Spécimens conservés en éthanol à $70^{\circ}$ dans la collection personnelle des auteurs (EI, MZ) ou dans celle du MNHN (Myriapoda, Lithobiomorpha) (JJG).

RÉPARTITION ET HABITAT. - Cette espèce, largement répartie en Europe occidentale et centrale, est commune en France et dans les Alpes-Maritimes (Iorio 2008, 2010b; Geoffroy \& Iorio 2009), à l'exception des zones circumméditerranéennes à basse altitude (Iorio 2014). F. Breton a récolté un spécimen de cette espèce (dét. E. Iorio) à l'altitude record de 3035 mètres à Saint-Paul-sur-Ubaye (04), à la Conque de Panestrel, en 2012. 29 des stations connues dans les AlpesMaritimes procurent des informations sur ses habitats : 18 d'entre elles sont des milieux forestiers (quatre lariçaies, deux châtaigneraies, deux forêts de feuillus dominées par le châtaignier, une châtaigneraie-corylaie, deux hêtraies-coniferes, deux forêts de conifères divers, une pinède, une pessière, une sapinière-pessière, une forêt de feuillus divers (ripisylve), une forêt sans autre précision), six des milieux ouverts alpins et subalpins (quatre prairies alpines et une prairie subalpine, une bordure de lac alpin) et cing des grottes ou avens. Cette dominance dans les boisements mésophiles à méso-thermophiles, tout en pouvant vivre en milieu ouvert à altitude élevée, correspondent à ses préférences écologiques connues dans les régions adjacentes (Geoffroy 1981b; Minelli \& Iovane 1987; Zapparoli 1993). Cependant, au regard d'une synthèse récente, on ne peut exclure qu'il y ait deux écotypes distincts au sein de cette espèce, dont un serait strictement sylvicole et l'autre subalpin à nival, au-dessus de la limite arborée (Iorio 2014).

\section{Lithobius (Lithobius) valesiacus Verhoeff, 1935}

Lithobius lusitanus valesiacus Verhoeff, 1935: 196.

LOCALITÉ TYPE. - Suisse, Twannklamm, 560 m d'altitude, audessus du lac Bieler.
Matériel EXAminé. - Beuil (06), PNM, Les Launes, conifères et lisière pelouse, $1600 \mathrm{~m}, 7 . V I .2008$, réc./dét. EI : $10^{7}$; Lucéram (06), Peira-Cava, La Cabanette, hêtres-conifères, 1350-1400 m, 1.X.2008, réc./dét. EI : $10^{\prime \prime}, 4$ \% ; Méolans-Revel (04), Tête de l'Estrop, 2961 m, 44,28652347N, 6,505249473E, 25.IX.2009, réc. FB/dét. EI : 1 웅 Saint-Martin-Vésubie (06), PNM, vallon Madone de Fenestre, lariçaie, $1830 \mathrm{~m}, 4^{\circ} 05^{\prime} 36^{\prime \prime} \mathrm{N}-7^{\circ} 21^{\prime 2} 24^{\prime} \mathrm{E}, 22 . V .2010$, réc./dét. MZ: $10^{\circ}$, 1 ; ; Saint-Martin-Vésubie (06), PNM, vallon Madone de Fenestre, milieux ouverts à proximité d'un boisement de genévriers, $1900 \mathrm{~m}$,

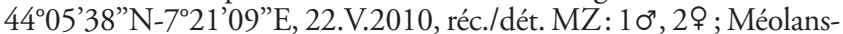
Revel (04), La Petite Barre, 2700 m, 44,28790484N, 6,527362399E, 24.IX.2010, réc. FB/dét. EI : 19 . Spécimens conservés en éthanol à $70^{\circ}$ dans la collection personnelle des auteurs (EI, MZ).

RÉPARTITION ET HABITAT. - $L$. ( $($ L.) valesiacus est un élément ouesteuropéen, présent aux Pays-Bas, en Allemagne, en Suisse, au Nord de l'Italie et dans le Nord-Est et le Sud-Est de la France (Massif vosgien; Massif alpin, Préalpes du Sud, Alpes maritimes) (Berg et al. 2008; Iorio 2008, 2010b). Il est exclusivement présent en France dans les régions montagneuses au moins dans le Sud-Est (Iorio 2008, 2010b), à partir de 1320 mètres d'altitude, et jusqu'à 2961 mètres (présent travail), ce qui constitue une altitude-record pour cette espèce. Ce caractère orophile se retrouve du côté italien (Minelli \& Zapparoli 1992; Zapparoli 1993). 16 des stations connues dans le département des Alpes-Maritimes renseignent sur l'habitat: huit milieux forestiers (quatre lariçaies, deux hêtraies-conifères, un boisement de conifères et sa lisière, et une forêt indéterminée), cinq prairies/ pelouses subalpines et alpines, une milieu ouvert à proximité de genévriers et deux en étage nival. Dans le Nord de sa répartition, $L$. (L.) valesiacus est sylvicole, de l'étage planitiaire à l'étage montagnard (Spelda 1999).

\section{Lithobius (Sigibius) microps Meinert, 1868}

Lithobius microps Meinert, 1868: 265.

LOCALITÉ TYPE. - Danemark, Copenhague.

MatéRIEl EXAMinÉ. - Menton (06), à proximité de la Gardieura, sentier GR 52, pinède et lisière de pinède, 350-400 m, 3.V.2013, réc./dét. EI : 1 \% ; Menton (06), à proximité du Chemin des Granges de Saint-Paul, dans une pinède, 400-450 m, 24.X.2013, réc./dét. EI : $10^{\pi}$ immature. Spécimens conservés en éthanol à $70^{\circ}$ dans la collection personnelle de l'auteur (EI).

RÉPARTITION ET HABITAT. - $-L$. $(S$.$) microps est largement réparti$ en Europe et en France (Geoffroy \& Iorio 2009). Bien que cette espèce euryèce soit commune au niveau national (Geoffroy \& Iorio 2009; Iorio 2010b, 2014), elle est moins fréquente dans les AlpesMaritimes, où peu d'informations sont disponibles sur les milieux des quelques stations connues dans le département (une près d'une grotte dans un vallon humide, une au bord d'un lac en montagne, une près d'une rivière et deux en pinèdes/lisières de pinèdes).

\section{Ordre SCOLOPENDROMORPHA Pocock, 1895 \\ Famille CRYPTOPIDAE Kohlrausch, 1881 Genre Cryptops Leach, 1815}

\section{Cryptops hortensis (Donovan, 1810)}

Scolopendra hortensis Donovan, 1810: 23.

LOCALITÉ TYPE. — Angleterre, Devon, Exeter, jardins.

Matériel eXAminé. - Saint-Martin-Vésubie (06), PNM, val-

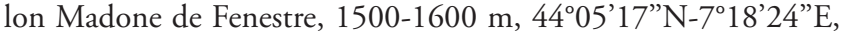


22.V.2010, réc./dét. MZ: 12 ex.; Nice (06), Parc Phénix, 8.I.2013, réc. JML/dét. EI : 1 ex. Spécimens conservés en éthanol à $70^{\circ}$ dans la collection personnelle des auteurs (EI, MZ).

RÉPARTITION ET HABITAT. - $C$. hortensis est très largement réparti, présent de l'Asie centrale à l'Europe, et il a même été introduit dans d'autres contrées, comme en Amérique du Nord (Zapparoli 2006; Iorio \& Geoffroy 2008). Il est connu dans de nombreux départements français, mais il est plus commun dans l'Ouest et le Centre du pays que dans l'Est (Iorio \& Geoffroy 2008; Geoffroy \& Iorio 2009). Dans les Alpes-Maritimes, il semble assez peu fréquent, à l'instar de ce qui s'observe ailleurs en France orientale et, même si peu de données écologiques sont disponibles, il a été trouvé aussi bien en milieu forestier qu'en milieu anthropisé (parcs urbains) (Iorio 2008; présent travail).

Cryptops lobatus Verhoeff, 1931

Cryptops lobatus Verhoeff, 1931: 280.

LOCALité TYPE. — France, Saint-Raphaël; Italie, Pegli.

MATÉRIEl eXAminé. - Sospel (06), PNM, grotte de l'Albaréa, 28.III.2009, réc. JML \& JR/dét. EI : 3 ex. ; Entrevaux (04), à proximité de Combe Guénier, 900-1000 m d'altitude, boisement de coniferes et de hêtres, près du secteur rocheux à profondes fissures, VII.2009, réc./dét. EI : 2 ex. Spécimens conservés en éthanol à $70^{\circ}$ dans la collection personnelle de l'auteur (EI).

RÉPARTITION ET HABITAT. - À l'opposé de C. hortensis et $C$. parisi Brölemann, 1920, C. lobatus possède une répartition nettement plus localisée, apparemment limitée à deux aires géographiques restreintes et disjointes. Il est présent dans une zone circumméditerranéenne allant de la Ligurie au littoral et reliefs préalpins des Alpes-Maritimes et de l'Est du Var, et du Sud-Est des Alpes-de-Haute-Provence (Iorio 2008; Iorio \& Geoffroy 2008; présent travail); il a été découvert dans l'arrière-pays Est-varois à Mons (83) (Ubac de Bliauge, pinèdes et leurs lisières, $1305 \mathrm{~m}$, $43,724366 \mathrm{~N}, 6,736831 \mathrm{E}, 15 . V .2012$, réc./dét. EI), et la mention d'Entrevaux constitue la première observation de cette espèce dans le département des Alpes-de-Haute-Provence. Elle existe également dans une petite zone pré-pyrénéenne au nord de l'Espagne (Vadell 2013). Ni l'intensité des prospections effectuées dans des départements intermédiaires entre les deux zones, notamment les Pyrénées-Orientales (cf. Brölemann 1926, 1930; Iorio 2014), ni les nombreuses études et révisions dont ont fait l'objet les espèces françaises du genre Cryptops (Brölemann 1925; Verhoeff 1931; Iorio \& Geoffroy 2004, 2007b, 2007c, 2008; Iorio \& Minelli 2005; Iorio 2010a), n'ont conduit à observer C. lobatus entre ces deux zones: l'hypothèse de l'existence de deux aires disjointes se trouve ainsi confortée. Neuf des stations connues dans le département des Alpes-Maritimes et ses environs informent sur l'habitat: deux forêts indéfinies; un boisement comportant chênes, pins et mimosas; une lisière de chênes en garrigue; un boisement de conifères et de hêtres; une pinède/lisière de pinède; un vallon boisé; une lisière forestière indéterminée et une grotte. En outre, la moitié sud du département des Alpes-Maritimes, qui regroupe l'essentiel des populations connues du territoire oriental et probablement global de cette espèce, est sujette à forte urbanisation. Les effets de l'avancée urbaine croissante, cause d'une stérilisation progressive des écosystèmes où vit cette espèce, peuvent assurément lui être néfastes au regard de ses préférences biotiques et abiotiques (nécessité de proies adaptées, affection des milieux humides et ombragés et de leurs micro-habitats favorables: repaires tels que pierres et rondins en contact étroit avec le sol terreux, couche inférieure de la litière) (Iorio 2006, 2008, 2014; Iorio \& Geoffroy 2008; présent travail).

\section{REMARQUES}

Quelques observations morphologiques viennent compléter les données connues (Iorio 2008; Iorio \& Geoffroy 2008) : les scies dentées des P. 21 sont dotées de $5+6,6+6$ à $7+7$ dents tibiales, et de $4+4$ à $5+5$ dents tarsales. La taille des individus s'échelonne de 12,5 à $14 \mathrm{~mm}$. Dès lors, en plus des caractères déjà connus et relativement fixes propres à ce taxon, les suivants présentent une variation intra-spécifique d'assez faible amplitude par rapport à d'autres espèces (Iorio \& Geoffroy 2008; présent travail) : taille 10 à 15 mm de long; scies dentées tibiales des P. 21 : six à dix dents, rarement cinq; scies dentées tarsales des P. 21 : quatre à six dents.

\section{Cryptops parisi Brölemann, 1920}

\section{Cryptops parisi Brölemann, 1920: 9.}

Localité type. — France, Côte-d’Or, Saint-Seine-l'Abbaye.

Matériel EXAMINé. - Allos (04), PNM, 11-17.VI.1899, réc. HWB/ dét. EI: 2 ex. ; Beuil (06), PNM, Les Launes, coniferes et lisière pelouse, 1600 m, 7.VI.2008, réc./dét. EI : 4 ex. ; Lucéram (06), Peira-Cava, La Cabanette, hêtres-coniferes, 1350-1400 m, 1.X.2008, réc./dét. EI : 1 ex. ; Saint-Dalmas-le-Selvage (06), PNM, vallon de Saint-Dalmas, forêt à dominante de mélèzes, 1400-1430 m, 15.VIII.2009, réc./ dét. EI : 3 ex. ; Saint-Dalmas-le-Selvage (06), PNM, près du refuge de Sestrière, conifères près d'une rivière, 2000 m, 16.VIII.2009, réc./dét. EI : 1 ex.; Saint-Dalmas-le-Selvage (06), PNM, La Buisse, lisière d'un bois, en surface, $1437 \mathrm{~m}$, lat. 44.2848357, 6.8875257E, 17.VIII.2009, réc./dét. JJG: 1 ex.; Saint-Dalmas-le-Selvage (06), PNM, forêt de Sestrière, mélèzaie, au sol, 1995 m, 44.2926706N, 6.8235944E, 17.VIII.2009, réc./dét. JJG : 1 ex. ; Colmars-les-Alpes (04), PNM, Ratery, forêt domaniale de la Haute-Vallée du Verdon, mélèzaie, sol et bois mort, $1700 \mathrm{~m}, 22$.VIII.2009, réc./dét. JJG: 3 ex.; Saint-Martin-Vésubie (06), PNM, Forêt de Boréon, parking 5, forêt mixte de conifères, $1500 \mathrm{~m}, 44^{\circ} 06^{\prime} 59^{\prime \prime} \mathrm{N}-7^{\circ} 16^{\prime} 44^{\prime \prime} \mathrm{E}$, 21.V.2010, réc./dét. MZ: 1 ex.; Saint-Martin-Vésubie (06), PNM, vallon Madone de Fenestre, ubac, $1600 \mathrm{~m}, 4^{\circ} 05^{\prime} 25^{\prime \prime} \mathrm{N}-7^{\circ} 19^{\prime} 53^{\prime \prime} \mathrm{E}$, 22.V.2010, réc./dét. MZ: 2 ex.; Valbonne (06), Sophia-Antipolis, lisières de pinèdes, $150 \mathrm{~m}, 43,638794 \mathrm{~N}, 7,044013 \mathrm{E}, 10 . \mathrm{IV} .2012$, réc./dét. EI : 1 ex.; Entrevaux (04), Combe Guénier, 12.XI.2013, réc. JML/dét. EI : 1 ex. Spécimens conservés en éthanol à $70^{\circ}$ dans la collection personnelle des auteurs (EI, MZ) ou dans celle du MNHN (Myriapoda, Scolopenmorpha) (JJG).

Répartition et habitat. - À l'instar de $C$. hortensis, $C$. parisi est très largement réparti dans toute l'Europe; en France, il est connu dans de nombreux départements, mais il est plus commun dans l'Est que dans le Centre et surtout que dans l'Ouest (Iorio \& Geoffroy 2008; Geoffroy \& Iorio 2009). Dans les Alpes-Maritimes, c'est de loin le scolopendromorphe le plus fréquent (Iorio 2008; présent travail), tout comme dans le Nord de l'Italie (Zapparoli \& Minelli 2006). 32 des stations connues dans le département des AlpesMaritimes (Iorio 2008; présent travail) montrent que $C$. parisi $\mathrm{y}$ vit essentiellement en milieu forestier varié, et dans une grande amplitude altitudinale (143 à 2400 mètres); 29 d'entre elles sont en effet forestières: six pinèdes et deux lisières de pinède/pelouse; quatre lariçaies; quatre forêts indéfinies; trois sapinières-pessières; deux pessières; deux châtaigneraies; deux hêtraies-coniferres; deux boisements mixtes de coniferes; une forêt de feuillus où domine le châtaignier; une lisière d'un bois indéterminé. trois autres stations sont ouvertes: une prairie alpine, une prairie subalpine, une lande montagnarde. 


\section{Cryptops trisulcatus Brölemann, 1902}

Cryptops biscarensis trisulcatus Brölemann, 1902: 73.

Localité type. - Pyrénées-Orientales, Port-Vendres.

Matériel eXAminé. — Guillaumes (06), PNM, Le Coulet, coniferes divers, 1470-1500 m, 8.VI.2008, réc.dét. EI : 1 ex. ; Valbonne (06), Sophia-Antipolis, pins, chênes verts et chênes pubescents, $236 \mathrm{~m}$, 43,625116N, 7,031673E, 11.IV.2012, réc./dét. EI: 1 ex.; Sospel (06), grotte de Sues, IV.2009, réc. JR \& JML/dét. EI : 1 ex. Spécimens conservés en éthanol à $70^{\circ}$ dans la collection personnelle de l'auteur (EI).

RÉPARTITION ET HABITAT. - La répartition de cette espèce est quasiment limitée aux régions méditerranéennes d'Europe, jusqu'en Turquie; elle existe également en Algérie et en Tunisie (Iorio \& Geoffroy 2008; Akkari et al. 2008). En France, C. trisulcatus n'est connu que des départements circumméditerranéens et en Corse (Iorio \& Geoffroy 2008; Zapparoli \& Iorio, 2012), où il est assez peu fréquent. Peu d'informations sont disponibles sur les habitats correspondant aux quelques stations connues dans le département des Alpes-Maritimes. Toutefois, les localisations des spécimens du côté français et les données écologiques italiennes (Minelli \& Iovane 1987; Zapparoli 2006; Zapparoli \& Iorio, 2012), confirment qu'il s'agit ici comme ailleurs d'une espèce thermophile surtout présente dans les formations arborées typiquement méditerranéennes.

\section{Cryptops umbricus umbricus Verhoeff, 1931}

\section{Cryptops umbricus Verhoeff, 1931: 283}

Localité type. - Italie, Umbria, Sud d'Arezzo; près de Pegli; Savona; Santaurio, Stressa au pied du Mont Mottarone.

Matériel examiné. - La Trinité (06), grotte du Plateau Tercier, VI.2008, réc. JR \& JML/dét. EI : 10 ex.; Roquebrune-Cap-Martin (06), grotte de Saint-Roman, 22.VII.2010, réc. JML/dét. EI : 1 ex.; Monaco, grotte du Trocadéro, 2012, réc. JML/dét. EI : 1 ex.; Monaco, dans les galeries souterraines est, réc. JML/dét. EI : 2 ex.; Peille (06), grotte de Morgelle (dite de "Saint-Jean »), 2012, réc. JML/dét. EI : 2 ex. Spécimens conservés en éthanol à $70^{\circ}$ dans la collection personnelle de l'auteur (EI).

RÉPARTITION ET HABITAT. $-C . u$. umbricus n'est présent que dans les Alpes-Maritimes et les Alpes-de-Haute-Provence en France, et assez largement réparti en Italie sans y être commun (Zapparoli \& Minelli 2006; Iorio \& Geoffroy 2008; Iorio 2010a; présent travail). On ne le trouve quasi-exclusivement que dans les grottes, y compris dans de petits avens peu profonds (Minelli 1985; Iorio \& Minelli 2005). Nous le qualifions ici d'eutroglophile en raison du fait que beaucoup de populations y sont établies en permanence, mais pour certaines, le milieu souterrain est très superficiel, voire artificiel. Lespèce présente des adaptations morphologiques nettement troglomorphes (allongement des articles antennaires, des antennes, et des pattes par rapport aux espèces épigées du même genre).

\section{REMARQUES}

Les spécimens examinés permettent de compléter la connaissance de certains caractères morphologiques: deux spécimens atteignaient $55 \mathrm{~mm}$ de long, deux autres $51 \mathrm{~mm}$ et $50,5 \mathrm{~mm}$; cette espèce peut donc excéder les $50 \mathrm{~mm}$ auparavant observés. La longueur des sillons céphaliques est toujours inférieure à $40 \%$ de celle de l'écusson céphalique chez les exemplaires étudiés (longueur $=26$ à $38 \%$ ), montrant la constance de ce caractère en cumulant avec tous les individus détaillés anté- rieurement (Iorio \& Minelli 2005; Iorio 2010a). Les scies dentées des P. 21 sont dotées de: $14+11,14+17,15+14$, $15+15,17+15,14+x, 12+x$ (immature) dents tibiales; $4+x, 4+4,4+5,5+5,5+6,3+x$ (immature) dents tarsales. Les amplitudes pour ces scies deviennent chez C. u. umbricus: 11 à 18 dents tibiales (neuf à dix chez les immatures, voire 12) et quatre à cinq dents tarsales (rarement trois = immatures).

\section{Cryptops umbricus lewisi Iorio, 2010}

Cryptops umbricus lewisi Iorio, 2010: 472.

LOCALITÉ TYPE. - Alpes-Maritimes, Gourdon, Aven du Fourchu.

MATÉRIEL EXAMiné. - Tende (06), hameau du Réfréi, Parc national du Mercantour, grotte, 1300-1400 m, 2009, réc. JML \& JR/dét. EI : 2 ex. ; idem, 2010: 2 ex. ; Cipières (06), massif de Calern, cavité Neva de Nrelac, été 2011, réc. JML/dét. EI : 1 ex.; Calern (06), aven de la Rougne, 2011, réc. JML/dét. EI : 1 ex.; aven du Col de Clavel (83), VI.2012-VII.2013, réc. JML \& JR/dét. EI : 1 ex. Spécimens conservés en éthanol à $70^{\circ}$ dans la collection personnelle de l'auteur (EI).

RÉPARTITION ET HABITAT. - Cryptops u. lewisi est endémique du SudEst de la France (Iorio 2010a) : il est connu dans les Alpes-Maritimes, et grâce à la présente étude, cité pour la première fois dans le département du Var. Nous le considérons également comme eutroglophile.

\section{REMARQUeS}

Notons que les antennes peuvent dépasser la longueur précédemment évoquée, en atteignant jusqu’à 5,5 fois la longueur de l'écusson céphalique, et le 10e article antennaire peut être jusqu’à quatre fois plus long que large. En revanche, la longueur des sillons céphaliques dépassent toujours $50 \%$ de celle de l'écusson céphalique, voire même plus de $60 \%$ (jusqu'à $65 \%$ observés); ces précisions ajoutent à la valeur discriminante de ce caractère (Iorio 2010a). Nous observons de 6 à 11 soies clypéales, et le bord rostral forcipulaire est de la forme décrite antérieurement (Iorio 2010a). Suite à l'observation de nouveaux spécimens, $C$. u. lewisi nous semble doté d'un nombre de dents tibiales moins important que celui du type aux P21: sept à dix dents chez des individus matures, tandis que ces nombres semblent être plutôt l'apanage d'immatures chez la forme typique. Les autres caractères propres à $C$. u. lewisi sont similaires à la description de Iorio (2010a). Précisons enfin que les deux sous-espèces n'ont encore jamais été trouvées ensemble dans une même cavité.

\section{Ordre GEOPHILOMORPHA Pocock, 1895 Famille HiMANTARIIDAE Bollman, 1893 Genre Stigmatogaster Latzel, 1880}

\section{Stigmatogaster gracilis (Meinert, 1870)}

\section{Himantarium gracilis Meinert, 1870: 32.}

LOCALITÉ TYPE. - Italie, Rome, Campagne romaine; Ariccia; Gennazzano, Ischia; Algérie, Bona; Espagne, Grenade. 
Matériel examiné. - Entrevaux (04), à proximité de Combe Guénier, 900-1000 m d'altitude, boisement de conifères et de hêtres, VII.2009, réc./dét. EI : 1 o à 93pp; Nice (06), parc de la Colline du Château, 23.III.2012, réc. JML/dét. EI : 19 à 95pp; Valbonne (06), Sophia-Antipolis, friches/lisières de pinèdes, $150 \mathrm{~m}$, $43,638794 \mathrm{~N}, 7,044013 \mathrm{E}, 10 . \mathrm{IV} .2012$, réc./dét. EI : 1 \% à $93 \mathrm{pp}$; Valbonne (06), Sophia-Antipolis, pins, chênes verts et chênes pubescents, $236 \mathrm{~m}, 43,625116 \mathrm{~N}, 7,031673 \mathrm{E}, 11 . I V .2012$, réc./ dét. EI : 1 \% à 95pp; Beausoleil (06), Giram, bosquets de chênes verts, 499 m, 43,754686N, 7,418105E, 23.IV.2012, réc./dét. EI : $20^{7}$ à 87 et 93pp; Roquebrune-Cap-Martin (06), hôtel Vista Palace, jardins en restanques et pinèdes, $210 \mathrm{~m}, 20 . V I .2012$, réc./dét. EI : 2 à 93pp; Nice (06), Pointe Madame, lisières de friches rudérales/ pinèdes, 40-50 m, 3.X.2012, 43,692375N, 7,307853E, réc./dét. EI : $20^{7}$ à 89 et $91 \mathrm{pp}$; idem, 4.X.2012: $10^{7}$ à $91 \mathrm{pp}$; Roquebrune-CapMartin (06), Mont Gros, pinède claire, 600-650 m, 31.X.2012, réc./ dét. EI : 1 o à 89pp, 1 ex. immature à 89pp; idem, 2.V.2013: 1 ex. immature à $91 \mathrm{pp}$; Menton (06), à proximité du Chemin des Granges de Saint-Paul, en lisière d'une pinède, 400-450 m, 24.X.2013, réc./ dét. EI : $10^{7}$ à $87 \mathrm{pp}$. Spécimens conservés en éthanol à $70^{\circ}$ dans la collection personnelle de l'auteur (EI).

RÉPARTITION ET HABITAT. - Cette espèce, limitée en Europe aux régions méditerranéennes de la Grèce à la France, existe également en Algérie et en Tunisie (Zapparoli 2006; Geoffroy \& Iorio 2009). En France, $S$. gracilis a été découvert pour la première fois dans le sud des Hautes-Alpes, ce qui constitue la limite septentrionale dans l'ouest de sa répartition (Lardier (05), entre Roméyère et La Garenne, clairière pierreuse près d'un boisement ripicole, $560 \mathrm{~m}, 44,407363 \mathrm{~N}$, 5,96613E, 20.VII.2011, réc./dét. EI). Dans les Alpes-Maritimes, il est connu de 0 à 1000 mètres d'altitude dans de nombreuses stations, surtout situées sur le littoral et les reliefs proches de celui-ci (Iorio 2008; présent travail). Des données écologiques sont connues pour une partie d'entre elles, elles indiquent une certaine ubiquité, des stations périurbaines à urbaines étant relativement anthropisées: quatre pinèdes dont une très clairsemée, deux oliveraies péri-urbaines, une chênaie-pinède, une forêt mixte de hêtres et conifères, un parc de château, quatre friches rudérales/lisières de pinède, un boisement mixte de pins, de chênes verts et de chênes pubescents, un bosquet de chênes verts, un jardin en restanques d'un hôtel. Du côté italien, $S$. gracilis est aussi considéré comme euryèce bien que lié aux milieux méditerranéens; il apparaît comme un taxon thermophile (Minelli \& Iovane 1987; Zapparoli 1993, 2006).

\section{Stigmatogaster dimidiata (Meinert, 1870)}

\section{Himantarium dimidiatum Meinert, 1870: 30.}

LOCALITÉ TYPE. — Espagne, Séville; Madère.

Matériel eXAMinÉ. - Roquebrune-Cap-Martin (06), Mont Gros, flanc d'exposition nord-est, chênaie claire/garrigue, 550-600 m, 30.X.2012, 43.7675N, 7.44138E, réc./dét. EI : $10^{7}$ à 117pp. Spécimens conservés en éthanol à $70^{\circ}$ dans la collection personnelle de l'auteur (EI).

RÉPARTITION ET HABITAT. - Il s'agit d'une espèce ouest-méditerranéenne, présente de l'Italie au Portugal et de la Tunisie au Maroc; en France, elle est rare et connue seulement dans les Alpes-Maritimes, les Pyrénées-Orientales et la Corse (Zapparoli 2006; Iorio 2008, 2014; Geoffroy \& Iorio 2009). Du fait de sa rareté, très peu de données écologiques sont connues dans les Alpes-Maritimes. En tenant compte des données connues du côté italien (Minelli \& Iovane 1987; Zapparoli 1993, 2006), S. dimidiata peut être considéré comme lié aux milieux thermophiles méditerranéens tels que les garrigues comportant des chênaies claires (les deux stations connues dans les Alpes-Maritimes sont, entre autres, de ce type) et les chênaies vertes.

\author{
Famille Dignathodontidae Cook, 1896 \\ Genre Dignathodon Meinert, 1870
}

Dignathodon microcephalus (Lucas, 1846)

Geophilus microcephalus Lucas, 1846: 288.

LOCALITÉ type. - Algérie, environs d'Oran, d'Alger, de Philippeville et du cercle de La Calle.

Matériel examiné. - Allos (04), PNM, 11-17.VI.1899, réc. HWB/dét. EI : $10^{7}$ à 71pp; Beuil (06), PNM, Les Launes, coniferes et lisière pelouse, 1600 m, 7.VI.2008, réc./dét. EI : $10^{7}$ à 73pp; Nice (06), parc de la Colline du Château, 18.XI.2011, réc. JML/dét. EI: $10^{7}$ à $71 \mathrm{pp}$. Spécimens conservés en éthanol à $70^{\circ}$ dans la collection personnelle de l'auteur (EI).

RÉPARTITION ET HABITAT. - Cette espèce est assez largement répartie en Europe méridionale jusqu'au Proche- et Moyen-Orient; au nord, elle remonte jusqu'en Autriche (Attems \& Würmli 1972). En France, D. microcephalus n'était connu quasiment que dans des départements du pourtour méditerranéen (Brölemann 1930; Iorio \& Berg 2007; Iorio 2008, 2014; Zapparoli \& Iorio 2012) et considéré comme xérophile (Geoffroy \& Iorio 2009), ce qui est assez rare chez les chilopodes. Nous l'avons trouvé dans les HautesAlpes (Réotier (05), en lisière rocailleuse de pinèdes et d'une pelouse xérophile, 950 m, 44,677208N, 6,602819E, 28.VII.2010, réc./dét. EI; La Roche de Rame (05), Chapelle de Rame, en lisière pierreuse de pelouses sèches, $930 \mathrm{~m}, 44,741501 \mathrm{~N}, 6,575972 \mathrm{E}, 20 . I V .2011$, réc./dét. EI). Dans les Alpes-Maritimes (Iorio 2008; présent travail), cette espèce est citée en garrigue (une station), en lisière d'une pelouse et de coniferes (une station), dans une forêt à dominante de châtaigniers (une station), dans le parc d'un château (une station) et "de préférence dans les terrains secs " par Caziot (1925). Généralement, elle vit de 0 à 1200 mètres d'altitude (Minelli \& Iovane 1987; Iorio 2014).

Genre Henia C. L. Koch, 1847

Henia (Chaetechelyne) vesuviana (Newport, 1844)

Geophilus vesuvianus Newport, 1844: 435.

LOCALITÉ TYPE. — inconnue.

Matériel eXaminé. - Lucéram (06), Peira-Cava, La Cabanette, hêtres-conifères, 1350-1400 m, 1.X.2008, réc./dét. EI : 1 O à 67pp; Sospel (06), grotte de Sues, IV.2009, réc. JR \& JML/dét. EI : $10^{\text {" }}$ à $67 \mathrm{pp}, 1$ à $69 \mathrm{pp}$; Entrevaux (04), Majastre, $560 \mathrm{~m}$, sous bois mort, 6.V.2009, réc. PF/dét. EI : 1 o à $83 p p$; Colmars-les-Alpes (04), PNM, Ratery, forêt domaniale de la Haute-Vallée du Verdon, mélèzaie, sol et bois mort, $1700 \mathrm{~m}, 22$.VIII.2009, réc./dét. JJG: 1 ㅇ S Saint-Martin-Vésubie (06), PNM, vallon Madone de Fenestre, milieux ouverts à proximité d'un boisement de genévriers, $1900 \mathrm{~m}$, $44^{\circ} 05^{\prime} 38^{\prime \prime} \mathrm{N}-7^{\circ} 21^{\prime} 09^{\prime} \mathrm{E}, 22 . V .2010$, réc./dét. $\mathrm{MZ}$ : 1 \% à $71 \mathrm{pp}$; Digne-les-Bains (04), Le Serre, $1100 \mathrm{~m}$, prairie humide, 27.V.2012, réc. $\mathrm{PF} /$ dét. $\mathrm{EI}: 10^{7}$ à $73 \mathrm{pp}$. Spécimens conservés en éthanol à $70^{\circ}$ dans la collection personnelle des auteurs (EI, MZ) ou dans celle du MNHN (Myriapoda, Geophilomorpha) (JJG).

RÉPARTITION ET HABITAT. — Cette espèce est assez largement répartie en Europe centrale et occidentale; elle est synanthrope au RoyaumeUni et au Pays-Bas (Zapparoli 2006; Barber 2009a). En France, $H$. vesuviana est commun dans de nombreux départements (Geoffroy \& Iorio 2009; Iorio 2014). Cette espèce, considérée comme euryèce (Minelli \& Iovane 1987; Zapparoli 2006), peut occuper un large spectre d'habitats, voire des habitats anthropisés tels que 
des parcs et jardins. Une préférence pour les forêts peut cependant être observée dans certaines régions frontalières (Zapparoli 1993), tout comme dans les Alpes-Maritimes (Iorio 2008; présent travail). Elle y est citée dans des pinèdes (six stations), des châtaigneraies (trois stations), des prairies montagnardes à subalpines (trois stations), des lariçaies (deux stations), des sapinières pessières (deux stations), des garrigues (deux stations), dans une aulnaie, une pessière, une hêtraie-conifères, une vallon boisé humide, deux forêts indéfinies, une milieu ouvert près de genévriers et dans une grotte. Au sein du département, elle existe de 0 à 2450 mètres d'altitude.

\section{Henia (Meinertia) bicarinata \\ (Meinert, 1870)}

Scotophilus bicarinatus Meinert, 1870: 41

LoCalité TYPe. - Italie, Gennazano, Campagne romaine; Sorrento; Tunisie, Tunis; Algérie, Bona; sud de l'Espagne.

RÉPARTition ET HABITAT. - Bosnie-Herzégovine, Bulgarie, Croatie, Espagne, France (Corse comprise), Grèce continentale et insulaire, Hongrie, Italie, Macaronésie; Afrique du Nord (Maghreb); Proche-Orient (Turquie, Caucase) (Zapparoli 2006; Geoffroy \& Iorio 2009). Dans les Alpes-Maritimes, signalé par Caziot (1925) à Cannes et sur les îles de Lérins, dans les cordons de varech; au Cap d'Antibes: plusieurs ex. dans chaque station; par Brölemann (1930) à l'île Saint-Honorat (une des deux îles de Lérins) (stations toujours dans les estrans d'après l'auteur). Espèce de chilopode vivant souvent (et parfois localement en très grand nombre) dans la zone intertidale et/ou dans ses abords immédiats (zone de mouillage par les vagues); occasionnellement, il peut aussi exister loin de la mer (Zapparoli \& Iorio 2012; Iorio 2014).

\section{REMARQUES}

Espèce non retrouvée malgré nos prospections sur le littoral de Cannes, sur celui du Cap d'Antibes et dans d'autres localités du littoral d'Ėze-Bord-de-Mer à Saint-Raphaël.

Famille SCHENDYLIDAE Cook, 1896

Genre Schendyla Bergsö \& Meinert, 1866

Schendyla carniolensis Verhoeff, 1902

\section{Schendyla carniolensis Verhoeff, 1902: 91.}

LOCALITÉ TYPE. - Adelsberg (Slovénie).

Matériel examiné. - Lucéram (06), Peira-Cava, La Cabanette,

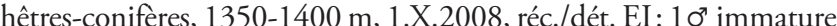
à 41pp; Menton (06), à proximité de la Gardieura, sentier GR 52, pinèdes et lisières de pinèdes, 350-400 m, 3.V.2013, réc./dét. EI : 19 à 49pp. Spécimens conservés en éthanol à $70^{\circ}$ dans la collection personnelle de l'auteur (EI).

RÉPARTITION ET HABITAT. - Schendyla carniolensis possède une répartition limitée au sein de l'Europe: Roumanie, Serbie, Slovénie, Autriche, Nord et Centre de l'Italie (très sporadique dans le sud du pays), France (Zapparoli 2006; Zapparoli \& Minelli 2006; Geoffroy \& Iorio 2009). En France, ce géophilomorphe est présent dans les Alpes-Maritimes et le Var, et de l'Aude aux Pyrénées-Atlantiques, tout en étant très sporadique à l'est des Pyrénées-Orientales (Iorio 2008, 2012, 2014). Dans les Alpes-Maritimes, cette espèce n'est citée que dans deux pinèdes (et/ou leurs lisières) et dans une forêt mixte de hêtres et de conifères, de 0 à 1400 mètres d'altitude (Iorio
2008 ; présent travail). Les données concernant le Nord et le Centre de l'Italie font apparaître que l'espèce est clairement sylvicole, privilégiant les forêts thermophiles et méso-thermophiles (Minelli \& Iovane 1987; Zapparoli 2006). Dans le Var, elle est également citée dans une forêt à dominante de hêtres (Iorio 2012). Autrefois citée « de Cannes à Menton " par Caziot (1925), il est possible qu' elle ait régressé sur cette partie du littoral en raison de l'importante croissance de l'urbanisation depuis les années 1920, et du fait qu'elle n'y ait pas été retrouvée ailleurs qu’à Menton.

\section{Famille GEOPHILIDAE Leach, 1815 Genre Geophilus Leach, 1815}

\section{Geophilus alpinus Meinert, 1870}

Geophilus alpinus Meinert, 1870: 76.

LOCALITÉ TYPE. — Italie, Razzes; Allemagne, Tübingen.

MatéRIEl EXAMINÉ. - Mandelieu (06), garrigue, en lisière arborée (chênes), 16 m, 43,528558N, 6,914716E, 7.V.2010, réc./dét. EI : 1 ex.; Saint-Martin-Vésubie (06), PNM, Forêt de Boréon, parking 5 ,

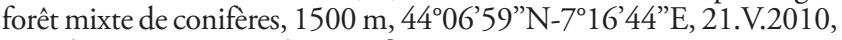
réc./dét. $\mathrm{MZ}: 10^{7}$ à $47 \mathrm{pp}, 1$ \% à $51 \mathrm{pp}$. Spécimens conservés en éthanol à $70^{\circ}$ dans la collection personnelle des auteurs (EI, MZ).

RÉPARTITION ET HABITAT. - La mise en synonymie de G. insculptus Attems, 1895 avec G. alpinus (Spelda 1999, 2005) est discutée au sein de la communauté myriapodologiste. Nous adoptons ici le second comme nom valide (cf. Zapparoli \& Iorio 2012; Iorio 2014). Celui-ci est largement réparti en Europe, et également présent en Tunisie et au Maroc; en France, il est aussi assez largement distribué mais semble absent du quart Nord-Ouest (Zapparoli 2006; Iorio 2014). Peu de stations sont connues pour G. alpinus dans les AlpesMaritimes, et de ce fait, peu de données écologiques sont disponibles dans ce département; les milieux sont connus uniquement pour les stations du présent travail. Toutefois, on peut le considérer comme relativement ubiquiste au vu des données connues dans les régions ou pays voisins (Minelli \& Iovane 1987; Zapparoli 1993, 2006; Iorio 2014).

\section{Geophilus carpophagus \\ Leach, 1815 sensu lato}

Geophilus carpophagus Leach, 1815: 384.

LOCALITÉ TYPE. - Inconnue.

Matériel eXAminé. - Lucéram (06), Peira-Cava, La Cabanette, hêtres-conifères, 1350-1400 m, 1.X.2008, réc./dét. EI : 1 O à 57pp; Sospel (06), PNM, grotte de Sues, IV.2009, réc. JR \& JML/dét. EI: 19 à 59pp; Entrevaux (04), à proximité de Combe Guénier, 900$1000 \mathrm{~m}$ d'altitude, boisement de coniferes et de hêtres, VII.2009, réc./dét. EI : $10^{\text {à }}$ a $3 \mathrm{pp}$; Menton (06), à proximité de Moniéri, mur le long de la petite route et de la lisière arbustive/arborée adjacente et près de quelques habitations en contrebas, $250-300 \mathrm{~m}$, 21.XI.2013, réc./dét. EI : $290^{7}$ (8 à 49pp, 21 à 51 pp), 9 우 (à $53 p p$ ). Spécimens conservés en éthanol à $70^{\circ}$ dans la collection personnelle de l'auteur (EI).

RÉPARTITION ET HABITAT. - En se basant sur le constat de Haswell et al. (2006) et celui, plus récent, de Bonato \& Minelli (2011), il est déraisonnable d'attribuer un statut spécifique précis à la plupart des populations européennes appartenant au " complexe " d'espèces de G. carpophagus (G. carpophagus s.s., G. easoni Arthur, Foddai, Kettle, Lewis, Luczinsky \& Minelli, 2001, et G. arenarius Meinert, 1870) en 
l'absence d'études spécifiques, en dehors de celles du littoral Atlantique, notamment du Royaume-Uni, pour $G$. carpophagus et $G$. easoni, et de celles d'Algérie pour $G$. arenarius. D'où la citation, dans le présent travail, de «G. carpophagus s.l. ». Ce « complexe » d'espèces est largement réparti en Europe et en France; il est présent jusqu'au Moyen-Orient (Brölemann 1930; Zapparoli 2006; Geoffroy \& Iorio 2009). 15 des stations connues dans les Alpes-Maritimes comportent des informations sur les habitats: trois pinèdes, trois hêtraies-coniferes, deux forêts indéterminées, une forêt de feuillus dominée par le châtaignier, une sapinière-pessière, une pessière, une garrigue parsemée de chênes, une prairie alpine et une subalpine, un mur le long d'une route, d'un talus bien végétalisé et de quelques habitations, et une grotte.

\section{Geophilus flavus (De Geer, 1778)}

Scolopendra flava De Geer, 1778: 561.

LOCALITÉ TYPE. - Inconnue.

MATÉRIEL EXAMINÉ. - Allos (04), PNM, 11-17.VI.1899, réc. HWB/dét. EI : 2 o à 51pp; Allos (04), PNM, bois de Villard, 16.VI.1899, réc. HWB/dét. EI : 1 9 à $51 \mathrm{pp}$; Beuil (06), PNM, Les Launes, conifères et lisière pelouse, 1600 m, 7.VI.2008, réc./ dét. EI : $20^{\top}$ à 49pp; Colmars-les-Alpes (04), PNM, Ratery, forêt domaniale de la Haute-Vallée du Verdon, mélèzaie, sol et bois mort, 1700 m, 22.VIII.2009, réc./dét. JJG: 1 우 ; Saint-Martin-Vésubie (06), PNM, Forêt de Boréon, parking 5, forêt mixte de conifères, $1500 \mathrm{~m}, 44^{\circ} 06^{\prime} 59^{\prime \prime} \mathrm{N}-7^{\circ} 16^{\prime} 44^{\prime \prime E}, 21 . V .2010$, réc./dét. MZ: 3 우 (1 9 à $51 \mathrm{pp}, 2$ à 53pp); Monaco, aven, XI-XII.2012, réc. JML/dét. EI : $10^{7}$ immature à 49pp; idem, I-III.2013: $10^{7}$ immature à $51 \mathrm{pp}$. Spécimens conservés en éthanol à $70^{\circ}$ dans la collection personnelle des auteurs (EI, MZ) ou dans celle du MNHN (Myriapoda, Geophilomorpha) (JJG).

RÉPARTITION ET HABITAT. — Ce géophilomorphe est largement réparti en Europe, également connu en Tunisie, et introduit dans diverses contrées, par exemple en Amérique du Nord; en France tout comme en Italie, il est connu dans de nombreux secteurs, et apparaît comme une des espèces les plus euryèces de cet ordre de chilopodes même s'il est plus souvent trouvé en forêt qu'ailleurs (Brölemann 1930; Minelli \& Iovane 1987; Zapparoli 2006; Geoffroy \& Iorio 2009; Iorio 2014). 20 des stations connues dans les Alpes-Maritimes informent sur les habitats: quatre forêts indéterminées, deux pessières, une pinède, une aulnaie, une hêtraie-conifères, une châtaigneraie et une forêt de feuillus dominée par le châtaignier, une mélèzaie, deux forêts de coniferes, une rhodoraie/prairie limitrophe, une prairie subalpine, une prairie montagnarde et une lisière de pelouse montagnarde, un milieu méditerranéen sur une île, et dans deux avens. G. flavus est présent de 0 à 2450 mètres dans ce département.

\section{Geophilus fucorum Brölemann, 1909}

Geophilus fucorum Brölemann, 1909: 359.

LOCALITÉ TYPE. - France, promontoire de Cannes-Croisette; îles de Lérins.

RÉPARTITION ET HABITAT. - France et Italie (littoral méditerranéen) (Geoffroy \& Iorio 2009; Minelli et al. 2013). Dans les AlpesMaritimes, signalé par Caziot (1925) et Attems (1929) à Cannes dans les cordons littoraux de varech et dans les îles de Lérins, où il était dit commun; par Brölemann $(190,1930)$ à île Saint-Honorat (une des deux îles de Lérins) et à Cannes-Croisette. Considéré par Brölemann (1930) comme présent plus largement sur le littoral des Alpes-Maritimes. Ce géophilomorphe vit exclusivement sur les grèves et dans les cordons de varech battus par les vagues (Brölemann 1930 ; Minelli \& Iovane 1987; Iorio 2014).

\section{REMARQUES}

Espèce non retrouvée malgré nos prospections sur le littoral de Cannes et Cannes-Croisette, ni dans d'autres localités du littoral d'Èze-Bord-de-Mer à Saint-Raphaël.

\section{Genre Pachymerium C. L. Koch, 1847}

\section{Pachymerium ferrugineum (C. L. Koch, 1835)}

Geophilus ferrugineus C. L. Koch, 1835: 2.

LOCALITÉ TYPE. - Allemagne, Regensburg, Arklee.

RÉpARTITION ET HABITAT. - Toute l'Europe, de la Scandinavie à l'Espagne et du Royaume-Uni jusqu'en Russie occidentale; Proche- et Moyen-Orient; Asie occidentale; Afrique du Nord; Alaska. Introduit en Amérique du Nord et centrale, également au Chili (Zapparoli, 2009b). Dans les Alpes-Maritimes, signalé par Brölemann (1904) à Monaco ; par Caziot (1925) sur tout le littoral de ce département, jusque dans les cordons de varech; par Brölemann (1930) à La Bocca (Cannes); par Iorio (2008) à Cannes la Bocca, plage, d'après un spécimen datant de 1905. Globalement, ce géophilomorphe est connu comme ayant une tendance halophile, parfois nette dans certains endroits (Brölemann 1930; Palmen \& Rantala 1954), sans être strictement inféodé aux habitats particuliers du bord de mer (Minelli \& Iovane 1987; Iorio 2014).

\section{REMARQUES}

Espèce non retrouvée malgré nos prospections sur le littoral d'Ezze-Bord-de-Mer à Saint-Raphaël, y compris à Cannes la Bocca.

\section{Genre Pleurogeophilus Verhoeff, 1901}

\section{Pleurogeophilus mediterraneus (Meinert, 1870)}

\section{Geophilus mediterraneus Meinert, 1870: 87.}

LOCALITÉ TYPE. — Italie, Bona.

Matériel eXAminé. - Lantosque (06), Loda, bord de rivière, pierres, 12.VI.2009, réc. SS/dét. JJG: 19 à 71pp; Duranus (06), cascade, dessous de pierres humides, 2009, réc. SS/dét. JJG: 1 \% à 73pp; Valbonne (06), Sophia-Antipolis, friches/lisières de pinèdes, $150 \mathrm{~m}, 43,638794 \mathrm{~N}, 7,044013 \mathrm{E}, 10 . \mathrm{IV} .2012$, réc./dét. EI: 1 \% à 73pp; Valbonne (06), Sophia-Antipolis, pins, chênes verts et chênes pubescents, $236 \mathrm{~m}, 43,625116 \mathrm{~N}, 7,031673 \mathrm{E}, 11 . I V .2012$, réc./ dét. EI : $10^{\prime 7}$ à $71 \mathrm{pp}$; Nice (06), Pointe Madame, lisières de friches et de pinèdes, $40-50 \mathrm{~m}, 3 . \mathrm{X} .2012,43,692375 \mathrm{~N}, 7,307853 \mathrm{E}$, réc./ dét. EI : $10^{7}$ à $71 \mathrm{pp}$; Monaco, aven, I-III.2013, réc. JML/dét. EI: $10^{7}$ à $71 \mathrm{pp}$; Menton (06), à proximité du Chemin des Granges de Saint-Paul, dans une pinède, 400-450 m, 24.X.2013, réc./dét. EI : 1 ex. immature à $73 \mathrm{pp}$. Spécimens conservés en éthanol à $70^{\circ}$ dans la collection personnelle de l'auteur (EI) ou dans celle du MNHN (Myriapoda, Geophilomorpha) (JJG).

RÉPARTITION ET HABITAT. - Ce géophilomorphe est formellement connu en Grèce, en Hongrie, dans le Nord de l'Italie, en France (uniquement dans les Alpes-Maritimes et l'Est du Var) et en Espagne (Baléares) (Attems 1929; Zapparoli \& Minelli 2006; Geoffroy \& Iorio 2009; Iorio 2014); sa répartition exacte reste imprécise (Zapparoli 2002). 15 des stations connues dans les Alpes-Maritimes renseignent sur les habitats: trois pinèdes, deux châtaigneraies, un vallon boisé humide, une pessière, une pinède/chênaie, une oliveraie, 
trois bordures de rivières, une garrigue avec chênes, une lisière de friches et de pinèdes et un aven. Les habitats préférentiels de cette espèce demeurent encore méconnus en France tout comme du côté italien, mais Zapparoli (2002) mentionne qu'en Grèce, l'espèce est plutôt euryèce. Dans les Alpes-Maritimes, nous constatons qu' elle est présente dans des milieux variés et parfois relativement anthropisés, péri-urbains. En revanche, elle n'est pas présente en haute montagne (altitude maximale connue: 1200 mètres) et semble être une espèce méso-méditerranéenne à supra-méditerranéenne.

\section{REMARQUES}

Brölemann (1930) a écrit que la concavité de la griffe forcipulaire est lisse chez cette espèce, alors qu'elle est en réalité finement crénelée, comme nous avons pu le voir sur les spécimens examinés à un grossissement de 100 fois (Iorio 2008).

Genre Stenotaenia C. L. Koch, 1847

Stenotaenia linearis (C. L. Koch, 1835)

Geophilus linearis C. L. Koch, 1835: 1.

Geophilus poseidonis Verhoeff, 1901: 421.

LOCALITÉ TYPE. - Allemagne, Regensburg.

Matériel eXaminé. - Nice (06), parc de la Colline du Château, 18.XI.2011, réc. JML/dét. EI : 2 \% à 69pp. Spécimens conservés en éthanol à $70^{\circ}$ dans la collection personnelle de l'auteur (EI).

RÉPARTITION ET HABITAT. - Stenotaenia linearis est largement réparti en Europe, bien qu'il soit essentiellement synanthropique dans le Nord et le Centre de l'Europe (Attems 1929; Brölemann 1930 ; Zapparoli 2006; Barber 2009a; Geoffroy \& Iorio 2009; Iorio 2014). Peu d'informations sont mentionnées sur les habitats de récolte dans les stations connues des Alpes-Maritimes, où il est avéré de 0 à 1450 mètres d'altitude: il n'est cité que dans une forêt de nature indéterminée, et dans un parc urbain. Cependant, cette espèce assez bien connue sur le plan écologique, est euryèce et fréquente aussi bien des boisements naturels que très artificiels comme les populicultures, ainsi que des milieux urbains et sub-urbains tels que parcs et jardins (Minelli \& Iovane 1987; Zapparoli 2006; Iorio 2014).

Genre Tuoba Chamberlin, 1920

Tuoba poseidonis (Verhoeff, 1901)

Geophilus poseidonis Verhoeff, 1901: 421.

LOCALITÉ TYPE. — Grèce, île Egine.

RÉPARTITION ET HABITAT. - Tuoba poseidonis est connu sur le littoral circumméditerranéen de la France à la Grèce. En Italie, les données sont surtout situées sur le littoral ouest, la Sicile et la Sardaigne. Il a aussi été recensé au bord de la Mer Rouge et de la Mer Morte (Zapparoli \& Iorio 2012). Dans les Alpes-Maritimes, il a été signalé par Caziot (1925) et Brölemann (1930) à l'île Saint-Honorat, dans les cordons de varech. Cette espèce méditerranéenne ne vit que le milieu étroit constitué par la zone intertidale et la zone de mouillage par les vagues, et n'est jamais trouvé au-dessus de cet ensemble. Ses micro-habitats sont, entre autres, les cordons littoraux de varechs et le dessous des posidonies échouées (Attems 1929; Brölemann 1930; Minelli \& Iovane 1987; Geoffroy \& Iorio 2009; Zapparoli \& Iorio 2012; Iorio 2014).
REMARQUES

Espèce non retrouvée malgré nos prospections sur le littoral d'Êze-Bord-de-Mer à Saint-Raphaël.

LiNOTAENIDAE Cook, 1899

Genre Strigamia Gray, 1843

Strigamia acuminata (Leach, 1815)

Geophilus acuminatus Leach, 1815: 386

LOCALITÉ TYPE. — Angleterre, Roborough Down près de Plymouth; champs de Battersea.

MATÉRIEL EXAMINÉ. - Saint-Martin-Vésubie (06), PNM, vallon Madone de Fenestre, lariçaie, $1830 \mathrm{~m}, 4^{\circ} 05^{\prime} 36^{\prime \prime N}-7^{\circ} 21^{\prime} 24^{\prime \prime} \mathrm{E}$, 22.V.2010, réc./dét. MZ: $10^{\pi}$ à 39pp. Spécimens conservés en éthanol à $70^{\circ}$ dans la collection personnelle de l'auteur (MZ).

RÉPARTITION ET HABITAT. - $S$. acuminata est largement réparti en Europe, mais est absent des contrées les plus nordiques (Norvège, Suède), sa limite septentrionale de répartition étant le sud du Danemark (Andersson et al. 2005; Zapparoli 2006; Geoffroy \& Iorio 2009; Bonato et al. 2012). Il est présent dans de nombreux départements français. Dans les Alpes-Maritimes, ce géophilomorphe est peu commun, connu en forêt et seulement dans les étages montagnards et sub-alpins (1350 à 2000 mètres d'altitude) : deux lariçaies, une pessière, une forêt de nature indéfinie. Notons que le caractère strictement sylvicole de celui-ci est déjà bien établi dans le reste de sa répartition, et qu'il s'agit d'une espèce essentiellement inféodée aux forêts caducifoliées, hêtraies notamment (Iorio 2014). Toutefois, à l'instar de $L$. (L.) dentatus évoqué plus haut, il semble aussi plus montagnard ici et peut parfois être trouvé dans d'autres types de boisements comme l'illustrent nos données et celles de Minelli \& Iovane (1987), Zapparoli (1993, 2006) et Spelda (1999), surtout dans le sud de sa répartition. Mais même dans cette dernière, les boisements caducifoliés et le hêtre en particulier restent notablement privilégiés; par exemple, sur les 73 stations où l'habitat est connu dans les Apennins centrales, 65 sont des forêts caducifoliées en tout ou partie ( 15 forêts mixtes), dont 43 hêtraies, et sept autres sont des forêts de conifères (sapinières, pinèdes) (Zapparoli 2006).

Strigamia crassipes (C. L. Koch, 1835)

Geophilus crassipes C. L. Koch, 1835: 3.

LOCALITÉ TYPE. - Allemagne, Regensburg.

MATÉRIEl eXAminé. - Saint-Dalmas-le-Selvage (06), La Buisse, lisière d'un bois, en surface, $1437 \mathrm{~m}, 44.2848357 \mathrm{~N}, 6.8875257 \mathrm{E}$, 17.VIII.2009, réc./dét. JJG: 19 à 57pp. Spécimens conservés en éthanol à $70^{\circ}$ dans la collection du MNHN (Myriapoda, Geophilomorpha) (JJG).

RÉPARTITION ET HABITAT. - $S$. crassipes est présent dans quasiment toute l'Europe, et il en est de même en France (Zapparoli 2006; Geoffroy \& Iorio 2009; Bonato et al. 2012). Dans les AlpesMaritimes, ce géophilomorphe est commun et, tout comme dans d'autres régions françaises (Iorio 2014), paraît assez peu exigeant sur le plan écologique, pouvant être trouvé dans des milieux variés, de 550 à 2400 mètres d'altitude: cinq pinèdes, quatre sapinièrespessières, trois forêts indéfinies, deux pessières, une sapinière, une forêt de feuillus dominée par le châtaignier, une lisière d'un bois, une rhodoraie et prairie limitrophe, trois prairies alpines et deux subalpines, une prairie montagnarde, et une lande thermophile. 
Strigamia transsilvanica (Verhoeff, 1928)

Scolioplanes transsilvanicus Verhoeff, 1928: 278.

LOCALITÉ TYPE. - Allemagne, forêts d'Hermann en Transylvanie; Gottschee, Krain; Alpes orientales; au sud d'Hermagor; Brenner; Tatra-Höhlenhain; Arlberg; Titisee en Forêt-Noire; Pilatus, Kulm.

Matériel examiné. - Saint-Martin-Vésubie (06), PNM, Forêt de Boréon, parking 5, forêt mixte de conifères, $1500 \mathrm{~m}$, $44^{\circ} 06^{\prime} 59^{\prime \prime} \mathrm{N}-7^{\circ} 16^{\prime} 44^{\prime \prime E}, 21 . V .2010$, réc./dét. MZ: $10^{\prime}$ à $45 \mathrm{pp}$, 29 à 49pp; Saint-Martin-Vésubie (06), PNM, vallon Madone de Fenestre, ubac, sapinière-pessière, près d'une petite rivière, $1700 \mathrm{~m}$, $44^{\circ} 05^{\prime} 26^{\prime \prime N}-7^{\circ} 20^{\prime} 48^{\prime \prime}$ E, 22.V.2010, réc./dét. MZ: $10^{\prime \prime}$ à $47 \mathrm{pp}, 1$ ㅇ à 51pp; Saint-Martin-Vésubie (06), PNM, vallon Madone de Fenestre, lariçaie, $1830 \mathrm{~m}, 4^{\circ} 05^{\prime} 36^{\prime \prime N}-7^{\circ} 21^{\prime 2} 24$ ”E, 22.V.2010, réc./dét. $\mathrm{MZ}: 2$ à 47 et $49 \mathrm{pp}$. Spécimens conservés en éthanol à $70^{\circ}$ dans la collection personnelle de l'auteur (MZ).

RÉPARTITION ET HABITAT. - Strigamia transsilvanica est une espèce centre-européenne, qui atteint au nord le centre de l'Allemagne, à l'est le Luxembourg, la Lorraine et la Provence-Alpes-Côte d'Azur; il n'est présent que dans le Nord de l'Italie (Spelda 1999; Zapparoli \& Minelli 2006; Geoffroy \& Iorio 2009). À l'est, il atteint les Carpathes qu'il occupe entièrement (Bonato et al. 2012). Dans les Alpes-Maritimes, ce géophilomorphe semble surtout présent en montagne (1000 à 2300 mètres d'altitude), alors que dans le NordEst de la France et en Allemagne, on peut le trouver à basse altitude (Spelda 1999; Iorio 2014). Parmi les habitats des stations connues figurent : trois lariçaies, deux sapinières-pessières, une sapinière, une forêt mixte de coniferres, une châtaigneraie, une forêt indéterminée, une rhodoraie, deux prairies alpines.

\section{REMARQUeS}

Chez les populations de Provence-Alpes-Côte d'Azur, par rapport à $S$. crassipes, $S$. transsilvanica semble posséder systématiquement une dépression sub-ovale à ovale médiane incolore sur les sternites, tandis que le premier est doté d'un sillon médian-longitudinal généralement foncé.

\section{DISCUSSION}

Près de 500 spécimens appartenant à 36 espèces de chilopodes ont pu être identifiés, apportant des données nouvelles très intéressantes pour les secteurs étudiés. En effet, une espèce souterraine provenant d'Entrevaux (04) est nouvelle pour la science: Lithobius (Lithobius) lemairei $\mathrm{n}$. sp.; sept espèces sont recensées pour la première fois dans l'enceinte du Parc national du Mercantour (cœur de parc + aire d'adhésion). Par ailleurs, six autres espèces ont été recensées pour la première fois dans des départements voisins de l'ensemble de l'aire géographique abordée dans la présente contribution. Dans ces départements, quatre de ces six espèces se trouvent en limite d'aire (limite orientale pour $L$. (L.) nicoeensis et Cryptops umbricus (avec la sous-espèce. $C$. u. lewisi); limite septentrionale pour $L$. (L.) pyrenaicus et Stigmatogaster gracilis). Par ailleurs, la signalisation d'Eupolybothrus fasciatus en France a été révisée et est considérée comme erronée.

Parmi les Geophilomorpha, des taxa n'ont jamais été trouvés au-dessus de la zone mouillée par les vagues ou au-dessus des laisses de mer selon les espèces, et ils vivent généralement plus bas, dans la zone située entre la marée haute et la marée basse (Brölemann 1930; Delamare-Deboutteville 1948; Lewis 1962; Binyon \& Lewis 1963; Minelli \& Iovane 1987; Barber 1992, 2009b; Zapparoli 2009b; GRETIA 2010; Iorio 2014), à l'image de Geophilus fucorum et de Tuoba poseidonis en Méditerranée. Ces espèces peuvent même se laisser submerger de nombreuses heures par l'eau de mer (Plateau 1890; Camus 1892; Cloudsley-Thompson 1948; Palmen \& Rantala 1954; Binyon \& Lewis 1963; Iorio 2006). Le terme d' " halobie» est donc le plus approprié pour ce groupe (= "Miriapodi halofili genuini » selon Silvestri 1903). Ces espèces sont très sensibles aux changements environnementaux et leur étude, ainsi que celle de l'ensemble de la biocénose de ces milieux, peuvent fournir des informations utiles sur l'état de conservation des littoraux (Silvestri et al. 2007; Ronci et al. 2010).

Malgré les prospections ciblées que nous avons menées à plusieurs reprises dans plusieurs des stations historiques tout comme dans d'autres localités du littoral d'Èze-Bord-de-Mer à Saint-Raphaël, nous ne sommes parvenus à retrouver aucune espèce halobie ( $G$. fucorum et $T$. poseidonis) ou halophile (Henia (Meinertia) bicarinata et Pachymerium ferrugineum) de geophilomorphes. Les fouilles dans les micro-habitats inspectés auraient pourtant dû donner de bons résultats, car ils sont propices à ces géophilomorphes (Brölemann 1930; Delamare-Deboutteville 1948; Lewis 1962; Minelli \& Iovane 1987; Barber 1992, 2009b; GRETIA 2010).

Ainsi, nos recherches ciblées sur les espèces halobies et halophiles de Geophilomorpha sur le littoral concerné montrent que ces taxa y ont au moins nettement régressé. Il faut souligner d'une part que l'état de conservation de leurs habitats et micro-habitats était souvent très mauvais en raison d'une forte anthropisation voire urbanisation, cette partie du littoral ayant subi de profondes modifications environnementales à partir de 1920-1930 (Daligaux 2003). D'autre part, peu de micro-habitats appropriés subsistaient même dans les parties non bétonnées et moins urbanisées, très probablement en raison d'un nettoyage et/ou nivellement outrancier(s) et précoce(s) dû $(s)$ au tourisme (exemples: peu de laisses de mer en dehors de Saint-Jean-Cap-Ferrat et d'Agay durant nos prospections; nivellement mécanique des plages de sable ou de cailloux fins conduisant au retournement voire écrasement des faces inférieures de galets et autres morceaux de bois humides, qui lorsqu' ils demeurent longtemps en place fixe, sont très favorables aux chilopodes). Seules les îles de Lérins, autres stations historiques au large de Cannes pour G. fucorum, H. bicarinata et T. poseidonis (Caziot 1925), qui n'ont malheureusement pas pu être prospectées, disposent de laisses de mer très bien conservées; elles recèlent d'ailleurs une abondante faune de coléoptères spécialisés (P. Ponel, inédit). Enfin, remarquons que sur le reste du littoral de Provence-Alpes-Côte d'Azur, nous ne sommes parvenus à récolter uniquement que $P$. ferrugineum dans une zone littorale salée (Iorio 2014), qui, des quatre taxons recherchés, est de loin le plus largement réparti au niveau européen et le moins exigeant, pouvant se trouver très loin des grèves (Minelli \& Iovane 1987; Iorio 2005, 2007; Zapparoli 2006). Les trois autres taxons n'ont été retrouvés nulle part dans cette région, malgré les recherches de deux des auteurs (EI et PP). 
TABLEAU 4. - Première liste d'espèces qualifiées de "sensibles » dans le Sud-Est de la France. Les motifs sont exposés à l'aide des références de Zapparoli \& Minelli (2006), lorio (2008, 2010a, 2010b, 2014), lorio \& Geoffroy (2008), Billi et al. (2011), Bonato et al. (2011, 2012), Vadell (2013) et du présent travail.

\begin{tabular}{|c|c|}
\hline Espèce & Motif \\
\hline $\begin{array}{l}\text { Lithobius fagniezi } \\
\quad \text { Ribaut, } 1926\end{array}$ & $\begin{array}{l}\text { Espèce endémique du Sud du département du Var, qui est à la fois : très localisée sur le plan surfacique, } \\
\text { et essentiellement dans une aire littorale de plus en plus urbanisée; écologiquement spécialisée, car troglobie. }\end{array}$ \\
\hline $\begin{array}{l}\text { Lithobius scotophilus } \\
\quad \text { Latzel, } 1887\end{array}$ & $\begin{array}{l}\text { Espèce endémique de l'Est du massif des Alpes maritimes, présente aux abords de la frontière franco-italienne, } \\
\text { rare et très localisée sur le plan surfacique, et écologiquement spécialisée car troglobie. }\end{array}$ \\
\hline Cryptops lobatus & Espèce localisée dans deux aires géographiques disjointes (littoral et reliefs des Préalpes de l'Est du Var à la \\
\hline & $\begin{array}{l}\text { Ligurie, à moins de } 1400 \text { mètres d'altitude; petite zone pré-pyrénéenne au nord de l'Espagne), globalement rare } \\
\text { et préférentiellement sylvicole, dont l'essentiel des populations est concentré dans la moitié littorale des Alpes- } \\
\text { Maritimes, soumise à forte urbanisation. }\end{array}$ \\
\hline $\begin{array}{l}\text { Henia bicarinata } \\
\text { (Risso, 1826) }\end{array}$ & $\begin{array}{l}\text { Espèce halophile méditerranéenne ayant régressé sur le littoral de Saint-Raphaël à Menton, et même disparu } \\
\text { dans plusieurs localités. }\end{array}$ \\
\hline $\begin{array}{l}\text { Clinopodes vesubiensis } \\
\text { Bonato, lorio \& Minelli, } \\
2011\end{array}$ & $\begin{array}{l}\text { Espèce endémique des Alpes maritimes françaises, présente des reliefs de la Vésubie jusqu'à Peira-Cava. En plus } \\
\text { de sa répartition géographique particulièrement restreinte, sa distribution altitudinale est également limitée } \\
\text { puisqu'elle est monticole (1130-2150 mètres d'altitude). Elle est également peu commune. }\end{array}$ \\
\hline $\begin{array}{l}\text { Geophilus fucorum } \\
\text { Brölemann, } 1909\end{array}$ & $\begin{array}{l}\text { Espèce halobie méditerranéenne ayant régressé sur le littoral de Saint-Raphaël à Menton, et même disparu dans } \\
\text { plusieurs localités; strictement inféodée à un habitat étroit aussi bien sur le plan écologique que surfacique (zone } \\
\text { intertidale et ses abords immédiats). }\end{array}$ \\
\hline $\begin{array}{l}\text { Tuoba poseidonis } \\
\text { (Verhoeff, 1901) }\end{array}$ & $\begin{array}{l}\text { Espèce halobie méditerranéenne ayant régressé sur le littoral de Saint-Raphaël à Menton, et même disparu dans } \\
\text { plusieurs localités; strictement inféodée à un habitat étroit aussi bien sur le plan écologique que surfacique (zone } \\
\text { intertidale et ses abords immédiats). }\end{array}$ \\
\hline $\begin{array}{r}\text { Strigamia cottiana } \\
\text { (Verhoeff, 1935) }\end{array}$ & $\begin{array}{l}\text { Espèce endémique d'un périmètre allant du sud des Alpes cottiennes aux Alpes maritimes, qui est très rare. } \\
\text { En plus de sa répartition géographique restreinte et de sa très faible fréquence, sa distribution altitudinale semble } \\
\text { également limitée puisqu'elle paraît monticole (1000-1950 mètres d'altitude). }\end{array}$ \\
\hline
\end{tabular}

À ce jour, la richesse spécifique en chilopodes du département des Alpes-Maritimes s'élève à 69 taxa formellement recensés et valides, tandis que celle du Parc national du Mercantour (cœur de parc + aire d'adhésion) atteint 37 espèces (Tableau 1). En tenant compte d'espèces présentes dans des communes contiguës à l'ensemble de cette aire, le total atteint 71 espèces (Tableau 1); on peut donc considérer que le territoire étudié dans la présente contribution contient quasiment la moitié des chilopodes répertoriés en France (Geoffroy \& Iorio 2009). De plus, six autres taxa douteux dans l'aire géographique considérée et/ou sur le plan taxonomique (Iorio 2008; L. Bonato comm. pers.), sont également cités dans cette aire: Lithobius (Lithobius) borealis Meinert, 1868, L. (L.) blanchardi Léger \& Duboscq, 1903 [= L. (L.) cassinensis Verhoeff, 1925: Iorio 2008], Cryptops sublitoralis Verhoeff, 1931, Hydroschendyla submarina (Grube, 1869), Geophilus pinivagus Verhoeff, 1928 et G. promontorii Verhoeff, 1928. Ils nécessiteront des recherches supplémentaires voire une révision des matériaux typiques selon les cas. Notons cependant qu'au vu de nos résultats cumulés à l'ensemble des précédentes contributions sur les Alpes-Maritimes et alentours, il est peu probable que $L$. $(L$.) borealis et $H$. submarina y soient effectivement présents. En effet, le premier n'a toujours pas été retrouvé malgré l'examen de nombreux matériaux, et nous supputons qu'au vu des confusions ayant été faites avec $L$. ( $L$.) lapidicola par les anciens auteurs, les mentions de présence de $L$. (L.) borealis dans l'aire étudiée, voire aussi plus au nord dans le Massif alpin, soient erronées. Le second, halobie et initialement considéré comme potentiel, ne s'y est peut-être pas ou guère maintenu. Enfin, Eupolybothrus excellens (Silvestri, 1894) (troglobionte) et E. tridentinus (Fanzago, 1874) ont été recensées dans des provinces italiennes voisines et pourraient, eux, peut-être exister de façon très sporadique dans le département des Alpes-Maritimes (Iorio 2008).
À l'instar de celui brièvement évoqué en introduction pour d'autres groupes (Médail \& Quézel 1997a, 1999; Médail \& Diadéma 2006; Billi et al. 2011), grâce au niveau de connaissance actuel, l'intérêt myriapodologique de l'aire biogéographique étudiée, Alpes maritimes au sens de la SOIUSA (Marazzi 2005) et plus largement Alpes maritimes et leurs abords immédiats (Sud des Alpes cottiennes, Préalpes de Nice, Est des Préalpes de Castellane, Ouest des Alpes ligures) apparaît tout-à-fait remarquable. En effet, outre la biodiversité évoquée plus haut, cette aire abrite six taxa endémiques, Lithobius (Lithobius) lemairei n. sp., L. (L.) scotophilus, Cryptops umbricus lewisi, Stigmatogaster arcisherculis (Brölemann, 1904), Clinopodes vesubiensis et Strigamia cottiana (Verhoeff, 1935), le premier, le troisième et le cinquième étant endémiques de France; et 22 autres espèces et sous-espèces s'y trouvent en limite d'aire de répartition (Tableau 1).

Par ailleurs, étant donné que d'une part, dans le Sud de la France, l'aire étudiée est aujourd'hui une des mieux connues concernant les chilopodes avec celle des Pyrénées et que, d'autre part, le département des Alpes-Maritimes est soumis à une importante anthropisation et urbanisation depuis des décennies, surtout sur son littoral aujourd'hui artificialisé à $90 \%$ (Daligaux 2003), il devient possible et nécessaire de définir un premier groupe d'espèces qui, selon nous, exigerait rapidement des mesures spécifiques d'étude, de suivi et de conservation. Nous qualifions ces espèces de "sensibles " de la façon suivante (Tableau 4): espèces particulières sur le plan de leur répartition géographique (= endémiques locales), voire altitudinale et suffisamment documentées sur ces aspects; espèces écologiquement spécialisées pour lesquelles une régression des populations a été constatée, à l'image des géophilomorphes halobies et halophiles abordés plus haut; espèces géographiquement localisées, non synanthropiques, 
qui présentent l'essentiel de leurs effectifs dans un territoire soumis à forte anthropisation/urbanisation (littoral des AlpesMaritimes et du Var, Préalpes de Nice, Est des Préalpes de Castellane). En s'appuyant sur ces critères simples mais selon nous justifiés, sept espèces des Alpes-Maritimes peuvent être soulignées comme sensibles dans cette première liste, ainsi qu'une endémique du département du Var qui est aussi très concernée (Tableau 4). L’identification de celles-ci, même si elle nécessite un examen sous loupe binoculaire (voire sous microscope pour certaines), est de difficulté modérée pour Lithobius (Lithobius) fagniezi, L. (L.) scotophilus, Henia bicarinata, Clinopodes vesubiensis, Geophilus fucorum et Tuoba poseidonis, voire même assez aisée pour Cryptops lobatus et Strigamia cottiana. Ainsi, même si une initiation préalable à la morphologie des chilopodes reste incontournable pour le naturaliste non-myriapodologiste, les difficultés d'identification ne constituent pas une barrière à la prise en compte de ces taxa de manière officielle.

\section{Remerciements}

L'inventaire biologique généralisé Mercantour / Alpi Marittime a été mis en place par le projet the European Distributed Institute of Taxonomy (EDIT) (2006-2011).

Nous remercions M.-F. Leccia (Parc National du Mercantour) et M. De Biaggi (Parco Alpi Marittime).

Nous tenons à vivement remercier les collègues qui, par leurs récoltes, leurs informations ou leur aide dans la recherche de certains documents, ont notablement contribué au présent article: Stéphane Bence, Lucio Bonato, François Breton, Louis Deharveng, Emmanuel Delfosse, Grégory Deso, JeanMichel Lemaire, Alessandro Minelli, Jean Raffaldi, Sandrine Salmon, Marielle Tardy et Augusto Vigna Taglianti. Nous sommes également reconnaissants envers Nesrine Akkari pour sa relecture et ses conseils.

\section{RÉFÉRENCES}

Aellen V. 1984. — Faune d'une grotte des Alpes-Maritimes. Mémoires de Biospéologie 9: 281-286.

AKKaRi N., STOEV P. \& LeWIS J.G.E. 2008. — The scolopendromorph centipedes (Chilopoda, Scolopendromorpha) of Tunisia: taxonomy, distribution and habitats. ZooKeys 3: 77-102.

Andersson G., Bjarne M., Scheller U., DJursvoll P., Budd G. \& GäRDENFORS U. 2005. - Mangfotingar. Myriapoda. Nationalnyckeln till Sveriges Flora och Fauna, Upsalla 351 p.

AtTems C. 1929. - Myriapoda 1: Geophilomorpha, in Schulze F. E. \& KüKenthal W. (eds), Das Tierreich, vol. 52. W. de Gruyter \& $\mathrm{C}^{\circ}$, Berlin \& Leipzig: 1-388.

AtTems C. 1930. - Myriapoda 2: Scolopendromorpha, in Schulze F. E. \& KüKenthal W. (eds), Das Tierreich, vol. 54. W. de Gruyter \& $\mathrm{C}^{\circ}$, Berlin \& Leipzig: 1-308.

Aтtems C. 1947. — Neue Geophilomorpha des Wiener Museums. Annalen des Naturhistorisches Museums in Wien 55: 50-149.

AtTems C. \& WÜRmLI K. 1972. — Klasse: Myriapoda. U-Klasse: Chilopoda. Catalogus faunae Austriae 11: 17-19.

BARBER A. D. 1992. - Distribution and habitat in British centipedes (Chilopoda), in Meyer E., Thaler K. \& SCHEdL W. (eds), Advances in Myriapodology. Proceedings of the 8th International Congress of Myriapodology. Berichte des Natur-
wissenschaftlich-Medizinischen Vereins in Innsbruck suppl. 10: 339-352.

BARBER A. D. 2009a. - Centipedes. Synopses of the British Fauna (New Series) No. 58, The Linnean Society of London, Field Studies Council, London 228 p.

BArber A. D. 2009b. - Littoral myriapods: a review. Soil Organisms 81 (3): 735-760.

Berg M. P., Soesbergen M., Tempelman D. \& Wijnhoven H. 2008. - Verspreidingsatlas Nederlandse landpissebedden, duizendpoten en miljoenpoten (Isopoda, Chilopoda, Diplopoda). EIS-Nederland, Vrije Universiteit-Afdeling Dierecologie, Amsterdam, 192 p.

Billi F., Bourgon A., Cornet M., Desriaux P., George G., Iorio E., RyMARCZyK F. \& VARENNE T. 2011. - Insectes, arachnides et myriapodes des Alpes-Maritimes : une faune riche, originale et vulnérable. Riviéra scientifique 100: 101-120.

BINYON J. \& LEWIS J. G. E. 1963. — Physiological adaptations of two species of centipede (Chilopoda: Geophilomorpha) to life on the shore. Journal of the Marine Biological Association of the United Kingdom 43: 49-55.

Bologna M. A. \& Vigna Taglianti A. 1985. - Fauna cavernicola delle Alpi Liguri. Annali del Museo civico di Storia naturale "Giacomo Doria » 84bis: 1-388.

Bonato L. \& Minelli A. 2011. - Geophilus arenarius, a longmisunderstood species in the still unresolved carpophagus speciescomplex (Chilopoda: Geophilidae). Zootaxa 3114: 40-49.

Bonato L., Barber A. \& Minelli A. 2006. - The European centipedes hitherto referred to Eurygeophilus, Mesogeophilus, and Chalandea (Chilopoda, Geophilomorpha): taxonomy, distribution, and geographical variation in segment number. Journal of Natural History 40 (7-8): 415-438.

Bonato L., Danyi L., Socci A. A. \& Minelli A. 2012. — Species diversity of Strigamia Gray, 1843 (Chilopoda: Linotaeniidae): a preliminary synthesis. Zootaxa 3593: 1-39.

Bonato L., IORIO E. \& Minelli A. 2011. — The centipede genus Clinopodes C. L. Koch, 1847 (Chilopoda, Geophilomorpha, Geophilidae): reassessment of species diversity and distribution, with a new species from the Maritime Alps (France). Zoosystema 33 (2): 175-205. http://dx.doi.org/10.5252/z2011n2a3

BRÖLEMANN H. W. 1901. - Matériaux pour servir à une faune des Myriapodes de France. Nouvelle série. La Feuille des Jeunes Naturalistes, (4) 31, $\mathrm{n}^{\circ} 371:$ 259-262.

BRÖLEMANN H.W. 1902. - Matériaux pour servir à une faune des myriapodes de France. Feuille des Jeunes Naturalistes, 32 : 73.

BRÖLEMANN H. W. 1904. - Chilopodes monégasques. Bulletin du Musée océanographique de Monaco 15: 1-15.

BRÖLEMANN H. W. 1909. — Quelques Géophilides nouveaux des collections du Muséum d'Histoire naturelle. Bulletin du Muséum d'Histoire naturelle, $6: 356-361$.

BrölemanN H. W. 1920. — Un nouveau Cryptops de France [Myriap.] : Cryptopsparisi n. sp. - Bulletin de la Société d'Histoire naturelle de Toulouse, 48 : 9-13.

Brölemann H. W. 1925. - Cryptops parisi Bröl. et Cryptops hortensis Leach. Bulletin de la Société d'Histoire naturelle de Toulouse 53: 192-196.

BrölemanN H. W. 1926. - Myriapodes des Pyrénées-Orientales. Bulletin de la Société d'Histoire naturelle de Toulouse, 55: 233-267.

BRÖLEMANN H. W. 1930. — Eléments d'une faune des myriapodes de France. Chilopodes. Faune de France, 25. P. Lechevalier, Paris, 405 p.

BRÖlemann H. W. \& RibauT H. 1911. — Diagnoses préliminaires d'espèces nouvelles de Schendylina [Myriap. Geophilomorpha]. Bulletin de la Société entomologique de France 10: 219-222.

Brölemann H. W. \& Ribaut H. 1912. - Essai d'une monographie des Schendylina (Myriapodes, Géophilomorphes). Nouvelles Archives du Muséum d'Histoire naturelle (5) 4 (1): 53-181, pl. 1-11.

CAmus F. 1892. - Note sur la présence de Geophilus (Schendyla) submarinus Grube et de quelques autres animaux marins sur la côte de Préfaille près Pornic (Loire-Inférieure). Bulletin de la Société des Sciences naturelles de l'Ouest de la France 1891: 21-34. 
Caziot C. 1915. - À propos d'une scolopendre rencontrée près d'Antibes. Riviera scientifique 2 (2): 3-5.

Caziot C. 1925. - Les Myriapodes du département des AlpesMaritimes et de la principauté de Monaco (Avec la contribution de H. W. Brölemann). Riviera scientifique 12 (1): 3-8.

Chalande J. \& Ribaut H. 1909. - Étude sur la systématique de la famille des Himantariidae (Myriapodes). Archives de Zoologie expérimentale et générale, 5e série 1 (2): 197-275.

ClOUdSLEY-ThOMPSON J. L. 1948. - Hydroschendyla submarina (Grube) in Yorkshire: with an historical review of the marine Myriapoda. The Naturalist 1948 (October-December): 149-152.

DaLIGAUX J. 2003 - Urbanisation et environnement sur les littoraux: une analyse spatiale. Rives méditerranéennes 15: 1-7.

Delamare-Deboutteville C. 1948. - Notes faunistiques sur les Myriapodes de Bretagne. L'Entomologiste 2 (4): 66-67.

Delaunay P. 2000. - Arthropodologie médicale des Alpes-Maritimes. Riviéra scientifique 84: 43-64.

Demange J.-M. 1955. - Myriapodes cavernicoles de France, avec la description d'une espèce et d'une variété nouvelles. Notes Biospéologiques 10: 191-200.

DEMANGE J.-M. 1958. - Sur quelques Myriapodes cavernicoles de France et de Suisse. Revue Suisse de Zoologie 65 (40) : 843-855.

DONOVAN E., 1810 - The Natural History of British Insects. London. 1810: $90 \mathrm{p}$.

EASON E.H. 1974. - The type specimens and identity of the species described in the genus Lithobius by F. Meinert and now preserved in the Zoological Museum, Copenhagen University (Chilopoda: Lithobiomorpha). Zoological Journal of the Linnean Society of London 55: 1-52.

FrÜND H.-C., BALKENHOL B. \& RUSZKOwsKi B. 1997. — Chilopoda in forest habitat-islands in north-west Westphalia, Germany. Entomologica Scandinavica supplement 51: 107-114.

GEER C. DE 1778. - Mémoires pour servir à l'histoire des insectes. Tome VII. P. Hesselberg, Stockholm, 1778: 950 p.

Geoffroy J.-J. 1981a. Myriapodes troglophiles des Causses Majeurs récoltés par le Spéléo-Club du Berry, in ASGC (eds), Grands Causses, 3. Amicale Spéléologique des Grands Causses, Millau: 181-195.

Geoffroy J.-J. 1981b. — Les Myriapodes du Parc national des Écrins. I. - Stations de récolte (1976-1979) et présentation générale des peuplements de Chilopodes et de Diplopodes. Travaux Scientifiques du Parc National des Écrins 1: 97-123.

GEOFFROY J.-J. 1982. - Quelques Arthropodes cavernicoles terrestres de la région des Causses Majeurs. Bulletin du Spéléo-Club des Causses 5: 59-72.

GeOFFrOY J.-J. \& IORIO E. 2009. — The French centipede fauna (Chilopoda): updated checklist and distribution in mainland France, Corsica and Monaco. Soil Organisms 81 (3): 671-694.

GERVAIS M. P. 1837. — Études pour servir à l'histoire naturelle des Myriapodes. Annales des Sciences naturelles, 2 (7) : 35-61.

GRETIA, 2010. - Inventaire des inÉertébrés continentaux des estrans rocheux et sableux de Basse-Normandie. Rapport pour la Région Basse-Normandie, l'Agence de l'eau Seine-Normandie, la DREAL de Basse-Normandie, le Conseil général de la Manche et le Syndicat mixte Calvados Littoral Espaces Naturels, 136 p.

Haswell M., ENGHOFF H. \& ARTHUR W. 2006. — Further studies on Geophilus carpophagus (sensu lato), and a reinterpretation of the structure of its labrum. Bulletin of the British Myriapod and Isopod Group 21: 2-7.

IORIO E. 2005. - Résultats de prospections myriapodologiques dans les forêts de Villers-Befey et d'Hémilly (57), et sur le site de la pelouse sableuse du Gründ (57-Puttelange-les-Thionville) (Myriapoda, Chilopoda). Bulletin de la Société Lorraine d'Entomologie 11: 23-25.

IORIO E. 2006. - La faune des Chilopodes du Massif Armoricain: biologie, liste préliminaire et détermination des espèces (Chilopoda). Mémoires de la Société linnéenne de Bordeaux 7: 1-73.
IORIO E. 2007. - A new contribution to the knowledge of centipedes of eastern France (Chilopoda). Schubartiana 2: 1-12.

IORIO E. 2008. - Contribution à l'étude des chilopodes (Chilopoda) des Alpes-Maritimes incluant une clé d'identification des lithobiomorphes Lithobiidae de Provence-Alpes-Côte d'Azur. Bulletin de la Société linnéenne de Provence 59: 127-190.

IORIO E. 2010a. - Description d'une nouvelle sous-espèce de Cryptops umbricus Verhoeff, 1931 (Chilopoda, Scolopendromorpha, Cryptopidae). Bulletin de la Société linnéenne de Bordeaux tome 144 (N.S.) 37 (4) (2009): 471-481.

IORIO E. 2010b. - Les Lithobies et genres voisins de France (Chilopoda, Lithobiomorpha). Révision de plusieurs espèces méconnues et nombreux apports inédits à la connaissance du genre Lithobius Leach, 1814. Avec une clé des familles, des genres et de toutes les espèces de l'ordre. Supplément à la Revue de l'Association Roussillonnaise d'Entomologie 19: 1-104.

IORIO E. 2012. - Première contribution à la connaissance des chilopodes (Chilopoda) de la hêtraie de la Sainte-Baume (Var). Bulletin de la Société linnéenne de Bordeaux tome 147 (N.S.), 40 (2): 235-241.

IORIO E. 2014. - Catalogue biogéographique des chilopodes (Chilopoda) de France métropolitaine. Mémoires de la Société linnéenne de Bordeaux 15: 1-372.

IORIO E. \& BerG M. P. 2007. — Première contribution à l'étude des chilopodes (Chilopoda) de Provence et description d'une nouvelle sous-espèce. Bulletin de la Société linnéenne de Provence 58: 21-36.

Iorio E. \& Geoffroy J.-J. 2004. — Étude des Scolopendromorphes français du genre Cryptops Leach, 1814. Première partie: Cryptops parisi Brölemann, 1920 et C. parisi var. cristata Ribaut, 1925 (Chilopoda, Scolopendromorpha, Cryptopidae). Le Bulletin de Phyllie 18 : 28-37.

IORIO E. \& GEOFFROY J.-J. 2007a. - Répartition géographique de Scutigera coleoptrata (Linné, 1758) en France (Chilopoda: Scutigeromorpha: Scutigeridae). Le Bulletin d'Arthropoda 30: 48-59.

IORIO E. \& GEOFFrOY J.-J. 2007b. — Étude comparative de quatre espèces du genre Cryptops Leach, 1815 (Chilopoda, Scolopendromorpha, Cryptopidae) en France. Le Bulletin d'Arthropoda 31: 29-35.

Iorio E. \& GeOffroy J.-J. 2007c. — Diagnose et répartition de trois Cryptops de France (Chilopoda, Scolopendromorpha, Cryptopidae). Le Bulletin d'Arthropoda 32: 26-38.

IORIO E. \& GEOFFROY J.-J. 2008. - Les scolopendromorphes de France (Chilopoda, Scolopendromorpha) : identification et distribution géographique des espèces. Riviéra scientifique 91 (2007): 73-90.

IORIO E. \& Minelli A. 2005. - Un Chilopode confirmé pour la faune de France: Cryptops umbricus Verhoeff, 1931 (Scolopendromorpha, Cryptopidae). Bulletin mensuel de la Société linnéenne de Lyon 74 (4): 150-157.

JEANNEL R. 1926. - Faune cavernicole de la France. Avec une étude des conditions d'existence dans le milieu souterrain. Paul Lechevalier, Paris VIe, 334 p.

Juberthie C., Bouillon M. \& Delay B. 1981. — Sur l'existence d'un milieu souterrain superficiel en zone calcaire. Mémoires de Biospéologie 8: 77-94.

Kime R. D. \& Iorio E. 2010. - Centipedes and Millipedes of Burgundy. Bulletin of the British Myriapod and Isopod Group 24: 15-30.

KoCH C. L. 1835. - Deutschlands Crustaceen Myriapoden und Arachniden, in (PAnZer G. W. F.) Herrich-SchäFfER G. A. W. Deutschlands Insecten. Pustet: 136.

$\mathrm{KocH}$ C. L. 1844. — Deutschlands Crustaceen Myriapoden und Arachniden in (PANZer G. W. F.) Herrich-SChäFfer G. A. W. Deutschlands Insecten. Pustet, Regensburg: 190.

Koch L. 1862. - Die Myriapodengattung Lithobius. Verlag von J. L. Lotzbeck, Nürnberg, 94 p.

Koren A. 1986. - Die Chilopoden - Fauna von Karnten und Osttirol. 1. Geophilomorpha, Scolopendromorpha. Carinthia II, 43. Klagenfurt, 87 p. 
LATZEL R. 1887. - Niovi Miriapodi della caverne di Liguria. Annali del Museo Civico di Storia Naturale di Genova 5 (2): 507-508.

LEACH W. E. 1815. - A tabular view of the external characters of four classes of animals, wich Linne arranged under Insecta; with the distribution of the genera composing three of these classes into orders, etc. and descriptions of several new genera and species. Transactions of the Linnean Society 11: 307-400.

LEWIS J. G. E. 1962. - The ecology, distribution and taxonomy of the centipedes found on the shore in the Plymouth area. Journal of the Marine Biological Association of the United Kingdom 42: 655-664.

LiNNAEus C. 1758. - Systema naturae. Editio Decima. Holmiae 1: $824 \mathrm{p}$.

Lock K., De Bakker D. \& De Vos B. 2001. — Centipede communities in the forest of Flanders. Pedobiologia 45: 27-35.

LUCAS H. 1846. - Note sur quelques nouvelles espèces d'insectes qui habitent les possessions francaises du Nord de l'Afrique. Revue zoologique 9: 283-289.

MANFREDI P. 1932. - Contributo alla conoscenza della fauna cavernicola italiana: Miriapodi. Rivista di Science Naturali "Natura " 23: 71-96.

Manfredi P. 1935. - V Contributo alla conoscenza dei Miriapodi cavernicoli italiani. Atti della Società Italiana di Scienze Naturali 74: 253-283.

Manfredi P. 1948. - VII contributo alla conoscenza dei Miriapodi cavernicoli. Atti della Società Italiana di Scienze naturali 87: 198-224.

MARAZZI S. 2005. - Atlante Orografico delle Alpi. SOIUSA. Priuli \& Verlucca editori, Pavone Canavese (TO), 416 p.

Matic Z. 1957. - Contributo alla conoscenza dei Chilopodi cavernicoli delle grotte d'Italia. Rassegna Speleologica Italiana 9 (2/3): 74-77.

Matic Z. 1960. — Die Cryptopiden (Myriapoda, Chilopoda) der Sammlung des Speologischen Institutes « E. Gh. Racovita » aus Cluj. Zoologischer Anzeiger 165: 442-447.

Matic Z. 1967. - Contribution à la connaissance des Lithobiides, Scutigérides et Cryptopsides des grottes de l'Italie. Fragmenta Entomologica 5 (1): 77-110.

Matic Z. 1968. - Contributo alla conoscenza dei Litobiidi (Lithobiomorpha-Chilopoda) di Spagna. Memorie del Museo Civico di Storia Naturale di Verona 16: 113-126.

MÉDAIL F. \& DiADÉMA K. 2006. — Biodiversité végétale méditerranéenne et anthropisation: approches macro et micro-régionales. Annales de Géographie 651: 618-640.

MÉDAIL F. \& QUÉZEL P. 1997. - Hot-spots analysis for conservation of plant biodiversity in the Mediterranean basin. Annals of the Missouri Botanical Garden 84: 112-127.

MÉDAIL F. \& QuÉzEL P. 1999. - Biodiversity hotspots in the Mediterranean basin: setting global conservation priorities. Conservation Biology 13: 1510-1513.

MEINERT F. 1868. — Dannmarks Scolopender og Lithobier. Naturhistorisk Tidsskrift 5 (3): 241-268.

MeinerT F. 1870. - Myriapoda Musaei Hauniensis. Bitrag til Myriapodernes morphologi og systematik. I. Geophile. Naturhistorisk Tidsskrift 7 (3): 1-128.

MeINERT F. 1872. Myriapoda Musaei Hauniensis. Bidrag til Myriapoderns Morphologi og Systematik. II. Lithobiini. Naturhistorisk Tidsskrift 8 (3): 281-344.

Minelli A. 1985. - Catalogo dei Diplopodi e dei Chilopodi cavernicoli italiani. Memori del Museo civico di Storia naturale di Verona (II serie), Sezione Biologica 4: 1-50.

Minelli A. 1992. - The Centipedes of North-Eastern Italy (Trentino-Alto Adige, Veneto, Friuli-Venezia Giulia) (Chilopoda). Gortania-Atti del Museo Friulano di Storia Naturale 13: 157-193.

Minelli A. \& Iovane E. 1987. - Habitat preferences and taxocenoses of Italian centipedes. Bollettino del Museo civico di Storia naturale di Venezia 37: 7-34.
Minelli A. \& Zapparoli M. 1985. — I Chilopodi della regione ligure con particolare riguardo al popolamento delle Alpi Liguri. Lavori della Società Italiana di Biogeografia n. s. 9: 373-411.

Minelli A. \& ZaPPAROLI M. 1992. — Considerazioni faunistiche e zoogeografiche sui Chilopodi delle Alpi occidentali. Biogeographia. Lavori della Società Italiana di Biogeografia n. s. 16: 211-243.

Minelli A., Bonato L. \& Dioguardi R. 2013. — Chilobase: a web resource for Chilopoda taxonomy. http://chilobase.bio.unipd.it/

Palmen E. \& Rantala M. 1954. - On the life-history and ecology of Pachymerium ferrugineum (C. L. Koch) (Chilopoda, Geophilidae). Annales zoologici Societatis Zoologica Botanica Fennica -Vanamo' 16 (3): 1-44.

Plateau F. 1890. — Les Myriapodes marins et la résistance des Arthropodes à respiration aérienne à la submersion. Journal de l'Anatomie et de Physiologie normales et pathologiques 26: 236-269.

Pocock R. I. 1890. - Res Ligusticae XI. Contributions to our knowledge of the Chilopoda of Liguria. Annali del Museo Civico di Storia Naturale di Genova, Ser. 2, vol. 9 (29): 59-68.

Ribaut H. 1915. - Notostigmophora, Scolopendromorpha, Geophilomorpha (Myriapodes) (1re série). Archives de Zoologie expérimentale et générale 55 (6): 323-346.

RISSO A. 1826. - Histoire naturelle des principales productions d'Europe méridionale, et particulièrement de celles des environs de Nice et des Alpes Maritimes. Tome cinquième. Imprimerie de Lachevardière, Paris, $400 \mathrm{p}$.

Ronci L., Paribelli S., PaVesi L., Zapparoli M. \& De Matthaeis E. 2010. - Il popolamento a invertebrati nella banquette a Posidonia oceanica del litorale laziale (La Frasca-Civitavecchia, Italia) in SIE (eds), Le Scienze Ecologiche Oggi, XX Congresso Società Italiana di Ecologia, Roma, 27-30.IX.2010, SIE, Roma: 41.

RŮŽIČKa V., Zacharda M., NĚMCOVÁ L., Šmilauer P. \& NeKola J. C. 2012. - Periglacial microclimate in low-altitude scree slopes supports relict biodiversity. Journal of Natural History 46 (35-36): 2145-2157.

SALINAS J. A. 1990. - Contribucion al conocimiento de los quilópodos de Navarra (Myriapoda: Chilopoda). Publicaciones de Biologia de la Universidad de Navarra, Serie Zoologica 20: 1-70.

SERRA A. 1980. - Contribución al conocimiento de los Lithobiomorpha (Chilopoda) de la Peninsula Ibérica. Facultad de Biologia, Universidad de Barcelona, Barcelona, 357 p.

Serra A. 1981. - Contribución al coneixment d'algunes subspècies de Lithobius pilicornis Newport (Chilopoda, Lithobiomorpha). Boletin de la Institucion Catalana de Historia Natural 47: 87-92.

Serra A. 1987. - Los Lithobius (Chilopoda, Lithobiomorpha) ibéricos con surcos latero-internos en los ultimos pares de patas. Descripcion de Lithobius longiscissus n. sp. Publicaciones del Departamento de Zoología, Universidad de Barcelona 13: 71-81.

Silvestri C., Campanaro A., De Matthaeis E., Iannilli V., PaVesi L. \& Zapparoli M. 2007. — Comunità di Artropodi nelle banquette a posidonia (Posidonia oceanica) del litorale tirrenico dell'Italia peninsulare, in UZI (eds), Riassunti dei contributi scientifici $68^{\circ}$ Congresso Unione Zoologica Italiana, Lecce 24-27 Settembre 2007. UZI, Lecce: 55.

Silvestri F. 1903. - Fauna Napoletana. Miriapodi viventi sulla spiaggia del mare presso Portici (Napoli). Annuario del Museo Zoologico della Regia.-Università di Napoli (N. S.) 1 (12): 1-5.

SPELDA J. 1999. - Verbreitungsmuster und Taxonomie der Chilopoda und Diplopoda Südwestdeutschlands. Diskriminanzanalytische Verfahren zur Trennung von Arten und Unterarten am Beispiel des Gattung Rhymogona Cook, 1896 (Diplopoda, Chordeumatida, Craspedosomatidae). Ph. D. Thesis, University of Ulm. Part I: 217 p. Part II: 324 p.

SPELDA J. 2005. - Improvements in the knowledge of the myriapod fauna of Southern Germany between 1988 and 2005 (Myriapoda: Chilopoda, Diplopoda, Pauropoda, Symphyla). Peckiana 4: 117-145. 
STÖCKLI E. 2009. - Literature-based survey on the Swiss fauna of Chilopoda. Soil organisms 81 (3): 647-669.

STOEV P. \& GeOfFroy J.-J. 2004. - An annotated catalogue of the scutigeromorph centipedes in the collection of the Muséum national d'Histoire naturelle, Paris (France) (Chilopoda: Scutigeromorpha). Zootaxa 635: 1-12.

Stoev P., Akkari N., Zapparoli M., Porco D., Enghoff H., Edgecombe G.D., Georgiev T. \& Penev L. 2010. - The centipede genus Eupolybothrus Verhoeff, 1907 (Chilopoda: Lithobiomorpha: Lithobiidae) in North Africa, a cybertaxonomic revision, with a key to all species in the genus and the first use of DNA barcoding for the group. ZooKeys 50: 29-77.

VADELl M. 2013. - Cryptops (Cryptops) lobatus Verhoeff, 1931 (Chilopoda: Scolopendromorpha: Cryptopidae), primera cita para la península ibérica. Boletin de la Asociacion Espanola de Entomologia 37 (1-2): 1-14.

VERHOEFF K. W. 1901. - Beiträge zur Kenntnis paläarktischer Myriopoden. XVI. Aufsatz: zur vergleichenden Morphologie, Systematik und Geographie der Chilopoden. Nova Acta Abhandlungen der Kaiserlichen Leopoldinisch-Carolinisch Deutschen Akademie der Naturforscher 77: 369-465.

VerHOEFF K. W. 1902. — Über Chilopoden von Südsteiermark Krain und Kroatien. Sitzungs-Berichte der Gesellschaft naturforschender Freunde zu Berlin: 90-93.

VerhoefF K. W. 1925. - Beiträge zur Kenntnis der Steinläufer, Lithobiiden. Archiv für Naturgeschichte 91: 124-160.

VERHOEFF K. W. 1928. — Geophilomorphen-Beiträge und eine Lithobius-Form. Mitteilungen aus dem Zoologischen Museum in Berlin 14: 229-286.

VerhoefF K. W. 1931. - Uber europäische Cryptops-Arten. Zoologischer Jahrbücher (Systematik) 62 (3): 263-288.

VERHOEFF K. W. 1934. - Beiträge zur Systematik und Geographie der Chilopoden. Zoologischer Jahrbücher (Systematik) 66: 1-112.

VerhoefF K. W. 1935. - Über Scolioplanes (Chilopoda). Zoologischer Anzeiger, $111: 10-23$
VerhoefF K. W. 1937. — Chilopoden-Studien. Zur kenntnis der Lithobiiden. Archiv für Naturgeschichte, N. F. 6 (2): 171-257.

Walckenaer C. A. \& Gervais P. 1847. - Histoire naturelle des Insectes Aptères. Tome quatrième. Librairie Encyclopédique de Roret, Paris, 4: 623 p.

Wolf B. 1938. - Animalium Cavernarum Catalogus. Vol. III. I. W. Junk, S-Gravenhage, $918 \mathrm{p}$.

ZAPPAROLI M. 1980. - Chilopodi Litobiomorfi epigei e cavernicoli delle Alpi occidentali (Chilopoda, Lithobiomorpha). Fragmenta Entomologica 15 (2): 281-294.

Zapparoli M. 1993. - Chilopodi dell'Italia Nord-Occidentale. Rivista Piemontese di Storia Naturale 14: 97-127.

Zapparoli M. 2002. - Catalogue of the Centipedes from Greece (Chilopoda). Fragmenta Entomologica 34 (1): 1-146.

ZAPPAROLI M. 2006. - A catalogue of the centipedes (Chilopoda) of Central Apennnines (Italy). Bollettino del Museo civico di Storia naturale di Verona 30: 165-273.

ZAPPAROLI M. 2009a. - Primi dati sul popolamento dei chilopodi delle riserve naturali "Agoraie di Sopra e Moggetto" (Liguria, Genova) e "Guadine Pradaccio" (Emilia-Romagna, Parma) (Chilopoda). Bollettino dell'Associazione Romana di Entomologia 64 (1-4): 39-58.

ZaPPAROLI M. 2009b. - An annotated catalogue of the epigeic and cave centipedes (Chilopoda) of Sardinia, in CERretti P., Mason F., Minelli A., Nardi G. \& Whitmore D. (eds), Research on the terrestrial arthropods of Sardinia (Italy). Zootaxa 2318: 56-168.

ZAPPAROLI M. \& IORIO E., 2012. - The centipedes (Chilopoda) of Corsica: catalogue of species with faunistic, zoogeographical and ecological remarks. International Journal of Myriapodology 7: 15-68.

Zapparoli M. \& Minelli A. 2006. - Chilopoda, in Ruffo S. \& STOCH F. (eds), Checklist and distribution of the Italian fauna. Memorie del Museo civico di Storia naturale di Verona 2. Serie: 123-125 + CD-ROM. 